\title{
Recognition of basic sorting motifs within synaptic membrane cargo proteins by the clathrin-adaptor complex AP-2
}

\author{
Dissertation \\ zur Erlangung des Doktorgrades \\ der Mathematisch-Naturwissenschaftlichen Fakultäten \\ der Georg-August-Universität zu Göttingen \\ vorgelegt von \\ Kathrin Kastning \\ aus Stadthagen
}

Göttingen, den 23. Mai 2005 
D 7

Referent: $\quad$ Prof. Dr. K. von Figura

Korreferent: Prof. Dr. G. Braus

Tag der mündlichen Prüfung 


\section{Erklärung an Eides Statt}

Hiermit versichere ich, daß die vorliegende Dissertation mit dem Titel

"Recognition of basic sorting motifs within synaptic membrane cargo proteins by the clathrin-adaptor complex AP-2"

selbständig und ohne unerlaubte Hilfe angefertigt wurde.

Göttingen, den 23. Mai 2005

Kathrin Kastning 
Table of contents

TABLE OF CONTENTS I

LIST OF FIGURES $\mathrm{V}$

ABBREVIATIONS VIII

DANKSAGUNG $\mathrm{X}$

1. Abstract 1

2. Introduction

2.1 Chemical synapses in the central nervous system 3

2.2 Architecture of the presynaptic compartment of excitatory synapses $\quad 4$

2.3 Architecture of the postsynaptic compartment of $\begin{array}{ll}\text { excitatory synapses } & 7\end{array}$

2.3.1 AMPA receptor trafficking during longterm potentiation 8

2.3.2 AMPA receptor trafficking during longterm depression 9

2.4 Synaptogenesis and establishment of neuronal polarity 13

2.4.1 Cell culture models for neuronal differentiation 15

2.4.2 Expression and sorting of synaptic vesicle proteins during $\begin{array}{ll}\text { neuronal differentiation } & 17\end{array}$

$2.5 \quad$ Clathrin-mediated endocytosis at the synapse 18

2.5.1 Recognition of cargo proteins during clathrin-mediated endocytosis 21

$\begin{array}{lll}2.6 & \text { Aims of the project } & 21\end{array}$

3. Material and methods

3.1 Materials

3.1.1 Plasmids, DNA constructs and oligonucleotides 26

2.1.2 Synthetic peptides used in for pulldown experiments 28

3.1.3 Antibodies and endocytosis ligands 28

3.2 Molecular biological methods

$\begin{array}{lll}3.2 .1 & \text { RNA isolation and RT-PCR } & 30\end{array}$ 
3.2.2 Annealing and phosphorylation of single stranded oligonucleotides

3.2.3 PCR amplification of DNA fragments 31

3.2.4 Colony PCR 31

3.2.5 PCR mutagenesis of plasmid DNA 32

3.2.6 Agarose gel electrophoresis of DNA fragments 32

3.2.7 Restriction digest of PCR fragments and vector 32

3.2.8 Dephosphorylation and ligation 32

3.2.9 Culture of E. coli strains and transformation into chemically competent cells 33

3.2.10 Isolation and purification of plasmid DNA 33

3.2.11 DNA sequencing 34

\subsection{Protein biochemical methods}

3.3.1 Expression and purification of GST-tagged proteins in the E. coli strain BL21

3.3.2 Expression and purification of $\mathrm{His}_{6}$-tagged proteins in E. coli BL21

3.3.3 Preperation of ratbrain extract 35

3.3.4 In-vitro-transcription / translation of proteins 35

3.3.5 Pulldown of proteins from ratbrain extract 36

3.3.6 Pulldown of purified $\mu 2 \Delta 157 \quad 36$

3.3.7 Pulldown of in-vitro-transcribed /translated proteins 36

3.3.8 SDS-PAGE and Western Blot analysis of proteins 37

3.4 Cell biological methods

3.4.1 Mammalian cell culture 38

3.4.2 Neuronal differentiation of cells in-vitro 38

3.4.3 Transient transfection of mammalian cells 39

3.4.4 Endocytosis assays in mammalian cells 40

3.4.5 Immunocytochemistry on fixed tissues 41 


\section{Results}

4.1 Characterization of the interaction between the GluR2 cytoplasmic tail and the AP-2 complex

4.1.1 The AMPA receptor subunit GluR2 cytoplasmic tail binds directly to $\mu 2$-adaptin of the AP-2 adaptor complex

4.1.2 A basic sorting motif in the AMPA receptor cytoplasmic tail mediates direct interaction with $\mu 2$-adaptin

4.1.3 Proteins from the pre- and postsynaptic site interact with $\mu 2$-adaptic via a basic sorting motif

4.1.4 Characterization of a putative binding site for basic sorting motifs in $\mu 2$-adaptin

4.1.5 Plasma membrane recruitment of soluble $\mu 2 \Delta 157$ by a synaptotagmin 1 chimera carrying the AMPA receptor basic sorting motif in transfected cells

4.1.6 Internalization of a chimeric construct from the plasma membrane of transfected cells is mediated by the GluR2 basic sorting motif

4.1.7 Depletion of $\mu 2$-adaptin results in a decreased internalization of the synaptotagmin 1 chimeric construct carrying the AMPA receptor basic sorting motif

\subsection{Sorting of SV proteins during neuronal differentiation}

4.2.1 Expression of genes encoding for synaptic vesicle proteins is switched on at different time points during neuronal differention of $\mathrm{P} 19$ cells

4.2.2 Synaptic vesicle proteins co-localize to the axon and a perinuclear compartment in P19 neurons

4.2.3 A perinuclear compartment containing synaptic vesicle proteins in P19 neuronal precursors does not originate from the Golgi 68

4.2.4 Analysis of a putative endosomal recycling compartment 70 containing synaptic vesicle proteins in neuronal precursor cells 


\section{Discussion}

5.1 The AMPA type glutamate receptor directly binds to the $\mu 2$-subunit of the clathrin-adaptor complex AP-2 via a dibasic sorting motif

5.1.1 A putative mechanism for the recognition of basic sorting motifs by $\mu 2$-adaptin

5.1.2 Interaction between the AMPA receptor cytoplasmic tail and $\mu 2$-adaptin facilitates clathrin-mediated endocytosis of the receptor in-vivo

5.1.3 A functional model for the role of the interaction between the AMPA receptor and the clathrin-adaptor complex in synaptic plasticity

5.2.1 Differential expression of synaptic vesicle proteins during neuronal differentiation may regulate de-novo synaptogenesis

5.2.2 A putative role for clathrin-mediated endocytosis in synaptic vesicle biogenesis

5.2.3 Targeted transport of active zone precursor vesicles to the forming synapse

5.2.4 A putative role for clathrin-mediated endocytosis of SV proteins at different stages of synaptogenesis

5.2.5 Retinoic acid-induced neuronal differentiation of embryonic precursor cells as a model system to characterize the de-novo SV biogenesis

6. Summary and conclusions 85

$\begin{array}{ll}7 . & 87\end{array}$

8. Lebenslauf 95 


\section{List of figures}

\section{Introduction}

Fig. 2.1 The chemical synapse

Fig. $2.2 \quad$ Protein markers of synaptic vesicles

Fig. 2.3 Exocytic and endocytic trafficking at the presynaptic active zone

Fig. 2.4 The cytoplasmic tail of heterotetrameric AMPA receptors interacts with various proteins at the postsynaptic active zone

Fig. 2.5 AMPA receptor trafficking during neuronal plasticity

Fig. 2.6 The cytoplasmic tail of the AMPA receptor subunit GluR2 binds various postsynaptic proteins that are involved in receptor trafficking

Fig. 2.7 Stages of development of primary hippocampal neurons in culture

Fig. 2.8 Pluripotent embryocarcinoma cells can be differentiated in-vitro into postmitotic neurons upon retinoic acid induction

Fig. $2.9 \quad$ Clathrin-mediated endocytosis of a transmembrane receptor

Fig. 2.10 Synaptic vesicle recycling at the presynaptic terminal

Fig. 2.11 Structural model of the AP-2 complex

Fig. 2.12 Structural model for a conformational change in the AP-2 complex that allows recognition of a tyrosine-based sorting motif at the plasma membrane

Fig. 2.13 Sequence alignment of three basic clusters

\section{Results}

Fig. 4.1 AP-2 and NSF from ratbrain extract bind to GST-GluR2 cytoplasmic tail

Fig. 4.2 The GluR2 cytoplasmic tail interacts directly with $\mu 2$-adaptin

Fig. 4.3 Recombinant soluble $\mu 2 \Delta 157$ can compete the pulldown of native AP-2 from ratbrain extract by GST-GluR2

Fig. 4.4 Sequence alignment of basic stretches in the AMPA receptor subunits GluR1, GluR2 and GluR3 
Fig. $4.5 \mu 2 \Delta 157$ directly binds to the cytoplasmic tails of different AMPA receptor subunits GluR1, GluR2 and GluR3

Fig. 4.6 Sequence of the AMPA receptor subunit GluR2 cytoplasmic tail

Fig. 4.7 Binding of $\mu 2$ to GluR2 depends on three basic residues K844, R845 and K847 within the GluR2 cytoplasmic tail

Fig. $4.8 \quad$ Alignment of basic sorting motifs

Fig. 4.9 The interaction between GluR2 and $\mu 2$ can be competed by a basic peptide comprising the synaptotagmin 1 binding site for $\mu 2$ adaptin

Fig. 4.10 Basic peptides derived from the AMPA receptor or synaptotagmin 1 basic sorting motifs compete the interaction between native AP-2 comlex and the GluR2 cytoplasmic tail

Fig. 4.11 Competion of the interaction between $\mu 2$ and GluR2 by a synaptotagmin1 derived peptide $(\mathrm{KR})$ is dependent on a dibasic motif

Fig. 4.12 Disruption of the pulldown of native AP-2 complex by GluR2 depends on two basic residues within a synthetic synaptotagmin 1 derived peptide $\mathrm{KR}$

Fig. 4.13 Competion of the interaction between $\mu 2$ and the GABA receptor subunit $\gamma 2$ by a synaptotagmin 1 derived basic peptide (KR)

Fig. 4.14 Protein sequence alignment of the $\mu 2$-adaptin subdomain $B$ with $\mu 1-, \mu 3-$ and $\mu 4$-adaptin

Fig. 4.15 Mutation of acidic patches on the $\mu 2$-surface does not disrupt binding to GluR2

Fig. 4.16 FLAG-Synaptotagmin 1 chimera carrying the AMPA receptor basic sorting motif

Fig. 4.17 A synaptotagmin 1 chimera carrying the AMPA receptor basic sorting motif pep $2 r$ recruits soluble $\mu 2 \Delta 157$ to the plasma membrane of transfected HEK 293 cells

Fig. 4.18 Internalization of HA-GluR2 from the plasma membrane of transfected Cos 7 cells

Fig. 4.19 The AMPA receptor basic sorting motif targets internalization of a chimeric construct from the plasma membrane of transfected cells 
Fig. 4.20 Quantitive analysis of FLAG-Syt1 $\Delta$ C2Bpep2r, pep2M, $\Delta$ C2B endocytosis in transfected Cos7 cells

Fig. 4.21 Specific depletion of $\mu 2$-adaptin in Cos7-cells

Fig. 4.22 Depletion of $\mu 2$-adaptin impaires clathrin-mediated endocytosis of a synaptotagmin 1 chimera carrying the AMPA receptor basic sorting motif

Fig. 4.23 Quantitative analysis of FLAG-Syt1 $\Delta$ C2Bpep2r in Cos7 cells depleted of $\mu 2$-adaptin

Fig. 4.24 Expression of genes encoding for synaptic vesicle proteins is switched on at different time points during neuronal differentiation of P19 cells

Fig.4.25 Synaptic vesicle proteins synaptobrevin 2, SV 2 and synaptotagmin 1 are expressed at different time points during neuronal differentiation of P19 cells

Fig.4.26 Synaptic vesicle proteins synaptobrevin 2, SV2, synaptotagmin 1 and synaptophysin co-localize in a perinuclear compartment and the axons of P19 neurons

Fig.4.27 Disruption of the Golgi apperatus does not affect the intracellular distribution of synaptobrevin 2 in P19 neuronal precursor cells

Fig.4.28 Internalized transferrin is targeted to the synaptic vesicle protein containing perinuclear compartment in P19 neuronal precursors

Fig.4.29 Synaptobrevin 2 does not localize to early endosomes in P19 neuronal precursor cells

Fig.4.30 Synaptobrevin 2 does not localize to a late recycling endosome in P19 neuronal precursor cells

Fig.4.31 Endocytosed synaptotagmin 1 is targeted to the perinuclear synaptobrevin 2 positive compartment in P19 neurons

\section{5 .Discussion}

Fig. 5.1 The two putative recognition sites for basic sorting motifs on the $\mu 2$-subdomain B are located to two acidic patches on opposite sides of the molecule

Fig.5.2 corresponding to Fig. 2E "AMPA receptor trafficking during neuronal plasticity" 


\section{Abbreviations}

\begin{tabular}{|c|c|}
\hline A & alanine \\
\hline a & adenosine \\
\hline AAK 1 & adaptor-associated kinase 1 \\
\hline $\mathrm{ABP}$ & AMPA receptor binding protein \\
\hline ADP & adenosine diphosphate \\
\hline AMPA & a-amino-3-hydroxy-5-methyl-isoxazole-propionic acid \\
\hline AMPA & a-amino-3-hydroxy-5-methyl-isoxazole-propionic acid \\
\hline AMPAR & a-amino-3-hydroxy-5-methyl-isoxazole-propionic acid receptor \\
\hline AP & adaptor protein \\
\hline APS & ammonium persulfate \\
\hline AR & a1B adrenergic receptor \\
\hline ARF 1 & ADP ribosylation factor \\
\hline ATP & adenosine triphosphate \\
\hline BNDF & brain derived neurotrophic factor \\
\hline bp & basepair \\
\hline BSA & bovine serum albumine \\
\hline C & cysteine \\
\hline C & cytosine \\
\hline $\mathrm{CaCl}_{2}$ & calcium chloride \\
\hline CaMKII & calcium / calmodulin kinase II \\
\hline $\mathrm{CCV}$ & clathrin-coated vesicle \\
\hline cDNA & complementary DNA \\
\hline $\mathrm{Cl}^{-}$ & chloride \\
\hline CME & clathrin-mediated endocytosis \\
\hline CNS & central nervous system \\
\hline $\mathrm{CO}_{2}$ & carbondioxide \\
\hline $\mathrm{D}$ & aspartate \\
\hline DMEM & Dulbecco's modified eagle medium \\
\hline DMSO & demethylsulfoxide \\
\hline DNA & desoxyribonucleic acid \\
\hline dNTP & desoxyribonucleic acid triphosphate \\
\hline EDTA & ethylendiaminetetraacetate \\
\hline ER & endoplasmic reticulum \\
\hline et al. & et alii \\
\hline $\mathrm{F}$ & bulky hydrophobic amino acid residue \\
\hline $\mathrm{F}$ & phenylalanine \\
\hline FCS & fetal calf serum \\
\hline G & glycine \\
\hline g & guanin \\
\hline GABA & g-aminobutiric acid \\
\hline GABAR & g-aminobutiric acid receptor \\
\hline GRIP & glutamate receptor interacting protein \\
\hline GSDB & goat serum dilution buffer \\
\hline GST & glutathione-S-transferase \\
\hline GTP & guanosin triphosphate \\
\hline h & hour \\
\hline HA & hemeagglutinine \\
\hline
\end{tabular}




\begin{tabular}{|c|c|}
\hline HEK 293 & human embryonic kidney \\
\hline HIP1 & huntingtin interacting protein 1 \\
\hline hsc 70 & heat shock chaperone 70 \\
\hline I & isoleucine \\
\hline $\mathrm{K}$ & lysine \\
\hline KAc & Potassium acetate \\
\hline kb & kilobase \\
\hline $\mathrm{kDa}$ & kilo Dalton \\
\hline $\mathrm{KIF} 1 \mathrm{~A}$ & kinesin superfamily protein $1 \mathrm{~A}$ \\
\hline $\mathrm{L}$ & leucine \\
\hline LB & Luria-Bertani \\
\hline LTD & longterm depression \\
\hline LTP & longterm potentiation \\
\hline M & methionine \\
\hline $\mathrm{M}$ & molar \\
\hline $\mathrm{MgSO}_{4}$ & Magnesium sulfate \\
\hline $\min$ & minute \\
\hline $\mathrm{ml}$ & mililiter \\
\hline $\mathrm{mM}$ & millimolar \\
\hline $\mathrm{mmol}$ & millimol \\
\hline mol & mol \\
\hline $\mathrm{N}$ & asparagine \\
\hline $\mathrm{NaAc}$ & sodium acetate \\
\hline $\mathrm{NaCl}$ & sodium chloride \\
\hline $\mathrm{NaOH}$ & sodium hydroxide \\
\hline $\mathrm{NCBI}$ & National Center of Biotechnology Information \\
\hline NGF & nerve growth factor \\
\hline $\mathrm{nm}$ & nanometer \\
\hline NMDA & N-methyl-D-aspartate \\
\hline NMDAR & N-methyl-D-aspartate receptor \\
\hline NSF & N-ethylmaleimide-sensitive fusion protein \\
\hline OD & optical density \\
\hline $\mathrm{P}$ & proline \\
\hline PAGE & plyacrylamide gel electrophoresis \\
\hline PBS & phosphate buffered saline \\
\hline PC 12 & pheochromocytoma \\
\hline PCR & polymerase chain reaction \\
\hline PenStrep & penicilline streptomycine \\
\hline PFA & paraformaldehyde \\
\hline $\mathrm{pH}$ & preponderance of hydrogene ions \\
\hline PICK & protein interacting with protein kinase $\mathrm{C}$ \\
\hline PPP 1 & Protein phosphatase 1 \\
\hline Q & glutamine \\
\hline $\mathrm{R}$ & arginine \\
\hline RIM & Rab interacting molecule \\
\hline RNA & ribonucleic acid \\
\hline rpm & rounds per minute \\
\hline RT-PCR & reverse transcription-polymerase chain reaction \\
\hline $\mathrm{S}$ & serine \\
\hline
\end{tabular}




\begin{tabular}{|c|c|}
\hline SDS & sodium dodecyl sulfate \\
\hline sec & second \\
\hline SiRNA & small interferring RNA \\
\hline SNAP & soluble NSF attachment proteins \\
\hline SNARE & soluble NSF attachment protein receptor \\
\hline Std. & standard \\
\hline SV & synaptic vesicle \\
\hline SVP & synaptic vesicle protein \\
\hline $\mathrm{T}$ & threonine \\
\hline t & thymine \\
\hline Taq & Thermus aquaticus \\
\hline TBS & tris buffered saline \\
\hline TE & Tris-EDTA \\
\hline TEMED & N,N,N,N-tetramethylethylendiamine \\
\hline Tris & Tris(hydroxymethyl)aminomethane \\
\hline U & units \\
\hline $\mathrm{u}$ & uracile \\
\hline UV & ultraviolet \\
\hline $\mathrm{v}$ & valine \\
\hline VAMP & vesicle associated membrane protein \\
\hline $\mathrm{x}$ & variable amino acid residue \\
\hline Y & tyrosine \\
\hline (Ptdlns)(3,4,5)P3 & phosphatidylinositol-(3,4,5)-trisphosphate \\
\hline$(P t d I n s)(4,5) P 2$ & phosphatidylinositol-(4,5)-bisphosphate \\
\hline$\mu \mathrm{Ci}$ & micro curie \\
\hline$\mu$ & microliter \\
\hline$\mu \mathrm{m}$ & micrometer \\
\hline$\mu \mathrm{M}$ & micromolar \\
\hline$\mu \mathrm{m}$ & micrometer \\
\hline umol & micromol \\
\hline $2 \mathrm{XYT}$ & twofold yeast extract-tryptone medium \\
\hline
\end{tabular}




\section{Abstract}

Communication in the central nervous system (CNS) occurs via chemical synapses, intercellular asymmetric junctions between an axon and the dendrite of a neighbouring neuron. Synapses comprised of a presynaptic release site and a postsynaptic receptive site. Internalization of synaptic proteins by means of clathrin-coated vesicles (CCVs) plays a major role in the regulation of processes at both sides of the synapse. At the presynaptic side synaptic vesicle proteins (SVP) and excess membrane are retrieved by clathrin-mediated endocytosis (CME).

At the postsynaptic active zone the strength of signal transmission can be modulated by clathrin-mediated internalization of neurotransmitter receptors. A pre-requisite for $\mathrm{CME}$ is the recognition of cargo proteins via sorting signals within their cytoplasmic portion by the heterotetrameric clathrin-adaptor AP-2 or other monomeric adaptors.

We provide evidence here that the medium subunit $\mu 2$ of the AP-2 complex interacts directly with the cytoplasmic tail of the excitatory AMPA receptor subunit GluR2 via a dibasic recognition motif. At the presynaptic site binding of $\mu 2$-adaptin to a dibasic cluster within the C2B-domain of the synaptic vesicle protein (SVP) synaptotagmin 1 provides a link to the endocytic machinery and is implicated in SV recycling.

We have used a chimeric approach to analyze the role of the AMPA receptor dibasic recognition motif in CME. The basic cluster was sufficient to facilitate clathrin-mediated internalization of a synaptotagmin 1 chimera carrying the AMPA receptor sorting motif from the plasma membrane of transfected cells. Inhibition of CME in cells transfected with siRNA directed against $\mu 2$-adaptin prevented internalization of the chimera.

Thus, recognition of the dibasic sorting motif by $\mu 2$-adaptin provides a direct link to the endocytic machinery, and is thereby involved in CME of excitatory AMPA type glutamate receptors similar to the recognition of the SVP synaptotagmin 1 by AP-2. This work shows that dibasic motifs function as a common recognition signal in the regulated internalization of membrane cargo proteins from both sides of the synapse.

Establishment of axonal polarity during neuronal differentiation requires the maturation of an intracellular sorting machinery that achieves directed transport of SVPs to the nascent synapse. In the second part of this work we have addressed the question if clathrin-mediated internalization from the plasma membrane of neuronal precursor cells plays a role in the formation of a SV precursor organelle.

Retinoic acid induced differentiation of unpolarized embryonic precursor cells into postmitotic neurons was used as a model system to study expression patterns of SVPs. The expression of SVPs is switched on at very early stages of neuronal differentiation that precede neurite outgrowth and synaptogenesis. SVPs reside within a perinuclear compartment in neuronal precursor cells. Colocalization of internalized transferrin with endogenous SVPS in the perinuclear compartment of neuronal precursor cells hints at an endosomal origin of the compartment, although no co-localization with markers for early or late endosomes was observed. An antibody feeding assay, in which a monoclonal antibody directed against the luminal domain of the SVP synaptotagmin 1 was internalized from the plasma membrane of postmitotic neurons and sorted into 
the perinuclear compartment, supports a putative recycling function of the compartment.

Our data support a model whereby clathrin-mediated cycling of SVPs between the plasma membrane and a perinuclear recycling compartment in neuronal precursor cells might be involved in the biogenesis of a SV precursor organelle that mediates transport of SVPs along the axon to the nascent synapse. 


\section{Introduction}

\subsection{Chemical synapses in the central nervous system}

Communication between neurons is made possible by chemical or electrical synapses, specialized cell-cell junctions. Electrical synapses communicate via gap junctions whereas cell-to-cell communication at chemical synapses functions via release of non-peptide neurotransmitters such as acetylcholine, glycine, GABA, glutamate, NMDA or AMPA (Fig. 2.1).

The presynaptic site is formed by an axon that makes contacts with the dendrite of neighbouring neurons. Arrival of an action potential at the presynaptic site results in an increase of the intracellular calcium concentration which triggers exocytosis of synaptic vesicles and thereby release of neurotransmitter into the synaptic cleft. Upon neurotransmitter receptor activation at the postsynaptic site membrane ion channels open and influx or efflux of ions leads to depolarization or hyperpolarization of the active zone plasma membrane.

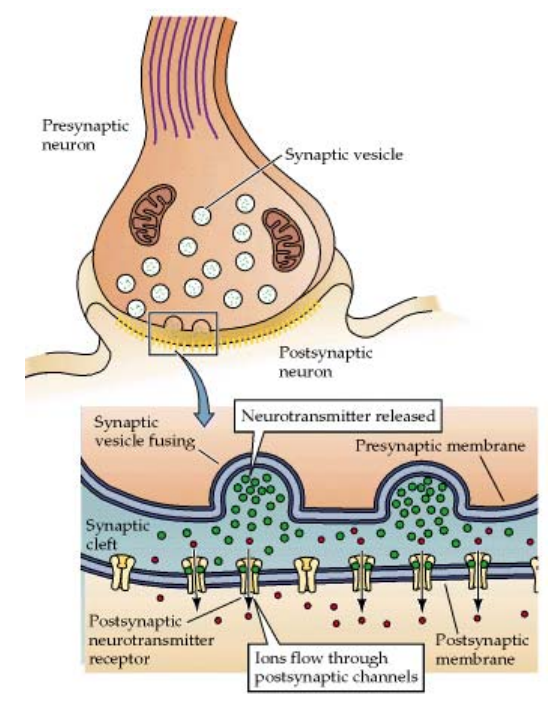

Fig. 2.1: The chemical synapse. Neurotransmitter release from synaptic vesicles at the presynaptic active zone results in activation of postsynaptic neurotransmitter receptors and ion flow through postsynaptic channels. Neuroscience, 2 nd edition, Sienauer, 2001 New York

The resulting postsynaptic potentials are either inhibitory or excitatory. Inhibitory synapses use GABA as neurotransmitter. GABA receptors are ligand gated ion channels which are selectively permeable to $\mathrm{Cl}^{-}$. Influx of $\mathrm{Cl}^{-}$drives hyperpolarization of the membrane resulting and thereby decreases the likehood of a postsynaptic action potential.

In contrast, the glutamatergic synapse mediates most of the fast excitatory transmission in brain using glutamate as neurotransmitter. Glutamatergic synapses comprise three types of neurotransmitter receptors including AMPA receptors, NMDA receptors and kainate receptors. Activation of AMPA receptors by glutamate results in $\mathrm{Ca}^{2+}$-influx and membrane depolarization thereby initiates neuronal firing. NMDA receptors have been implicated in synaptic plasticity mechanisms.

Synaptic plasticity can be induced by changes in the number of postsynaptic receptors. In hippocampal longterm depression AMPA receptors become 
internalized from the postsynaptic plasma membrane by clathrin-mediated endocytosis. At the presynaptic terminal clathrin-mediated endocytosis plays an important role in synaptic vesicle recycling.

The following chapters will outline the architecture of the pre- and postsynaptic compartment and introduce clathrin-mediated endocytosis as a common mechanism of protein trafficking at synaptic compartments.

\subsection{Architecture of the presynaptic compartment of excitatory synapses}

A synapse comprises a specialized intercellular junction between a presynaptic release site and a postsynaptic receptive site. The presynaptic active zone is a specialized exocytic site that faces the synaptic cleft. It is characterized by the presence of an electron dense structure adjacent to the plasma membrane. This presynaptic grip is a protein scaffold in which synaptic vesicles (SVs) are embedded (Sudhof, 2004; Murthy \& deCamilli, 2003). The hexagonal grid is formed by several large non-membrane proteins that bind to each other including members of the RIM (Rab 3 interacting molecule) and piccolo and bassoon families (Murthy \& deCamilli, 2003). RIM interacts with the small GTPase Rab3 present on the plasma membrane of SVs and might thereby function in tethering SVs to the active zone. Synaptic vesicles are small round organelles of a uniform diameter around $40 \mathrm{~nm}$ which can fuse with the presynaptic plasma membrane upon stimulation and calcium influx to release neurotransmitters into the synaptic cleft.

SVs reside in different pools at the presynaptic active zone: The readily releasable pool is comprised of a few vesicles docked to the active zone plasma membrane that are primed for immediate neurotransmitter release upon stimulation (Rizzoli \& Betz, 2005; DeCamilli et al., 2001). SVs clustering further away from the plasma membrane form the recycling pool, that maintains release during moderate stimulation, and the reserve pool, that is only exocytosed during intense stimulation (Rizzoli \& Betz, 2005).

Given that a $40 \mathrm{~nm}$ synaptic vesicle contains 10000 molecules of phospholipids with a combined molecular weight of proteins between $5000 \mathrm{kDa}$ to $10000 \mathrm{kDa}$ a maximum of 200 protein molecules of an average molecular weight of $50 \mathrm{kDa}$ are present in the vesicle. Based on their function these proteins can be divided into two classes: The first class are transport proteins which execute the uptake of neurotransmitters and other components into the lumen of the vesicle. The second class consists of trafficking proteins which function in plasma membrane targeting and are involved in either exocytosis or endocytosis of the synaptic vesicle (fig. 2.2).

Synaptotagmin 1 is an abundant synaptic vesicle glycoprotein that contains two $\mathrm{Ca}^{2+}$-binding C2-domains and functions as a calcium sensor in exocytosis of synaptic vesicles (Sudhof \& Rizo, 1996). By interacting with the AP-2 adaptor complex via a basic sorting motif, perhaps other adaptors and plasma membrane phospholipids it promotes synaptic vesicle recycling (Zhang et al., 1994; Liu et al., 1995; Jorgensen et al., 1995; Llinas et al., 2004; NicholsonTomishima \& Ryan, 2004).

The vesicle associated membrane protein VAMP / synaptobrevin 2 forms part of the SNARE-complex. It interacts with the plasma membrane associated tSNARE proteins syntaxin and SNAP25 and thus participates in synaptic vesicle 
exocytosis (Jahn \& Sudhof, 1999). Synaptobrevin also forms a complex with synaptophysin which is up regulated during neuronal development (Becher et al., 1999).

Synaptophysin is an abundant synaptic vesicle protein of unknown function. It interacts with synaptobrevin 2 and binds to cholesterol (Thiele et al., 1999).

SV 2 is a highly glycosylated protein containing 12 transmembrane domains. It shares homology with mammalian ion and sugar transporters though its transport function remains yet undescribed. SV2B forms complexes with synaptotagmin 1 in a $\mathrm{Ca}^{2+}$ independent manner (Lazzell et al., 2004).

The synaptic vesicle proteins synapsin 1, 2 and 3 function as ATPases (Hosaka \& Sudhof, 1998). They form oligomers and bind in a phosphorylation regulated manner to actin filaments (Greengard et al., 1993) consistent with their roles in synaptic vesicle clustering and actin-dependent guidance of synaptic vesicle recycling (Bloom et al., 2003).

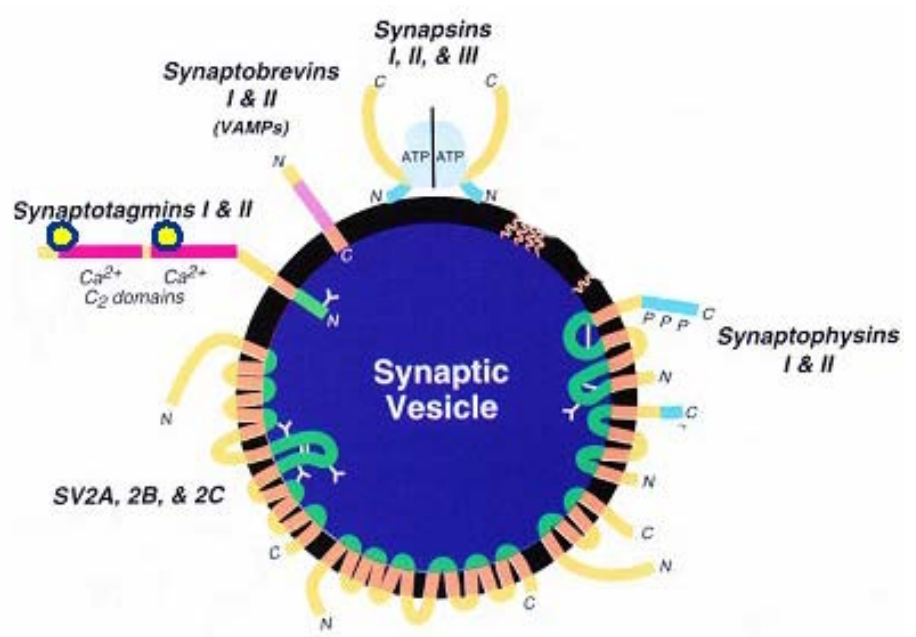

Fig. 2.2: Protein markers of synaptic vesicles. Modified from Sudhof, 1995

Vesicles docked to the active zone plasma membrane have to undergo a priming procedure to become fusion competent for exocytosis and neurotransmitter release. Interaction between vesicular and plasma membrane proteins and lipids play a role in exocytosis (see fig. 2.3).

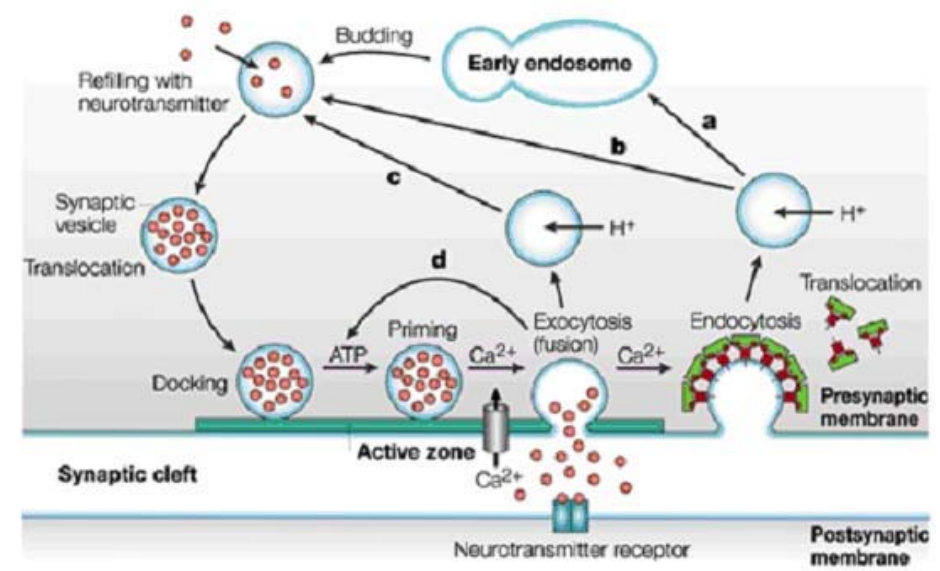

Fig. 2.3: Exocytic and endocytic trafficking at the presynaptic active zone. Before calcium-dependent fusion and neurotransmitter release SVs undergo docking and fusion steps. Taken from Gundelfinger et al., 2003 
The SNARE complex represents the minimal machinery for fusion (Murthy \& deCamilli, 2003;; Weber et al., 1998). SNARE proteins share a common SNARE motif comprised of 70 residues. The core complex is formed of four SNARE motifs of three or four seperate SNARE proteins that assemble into a four-helix bundle (Sudhof, 2004). The neuronal SNARE complex consists of the vesicular SNARE protein synaptobrevin / VAMP (vesicle associated membrane protein) 2 and the plasma membrane SNARE proteins syntaxin and SNAP 25 (Söllner et al., 1993). SNARE complex formation pulls the synaptic vesicle and plasma membrane close together and thereby creates a fusion intermediate (Sudhof, 2004).

SNARE function in exocytosis is controlled by SNARE interacting proteins such as munc 13, munc 18 and Rab 3 (Murthy \& deCamilli, 2003; Gundelfinger et al., 2003).

The SNARE protein syntaxin contains an N-terminal three-helix domain that can bind to its SNARE motif resulting in a closed conformation (Murthy et al., 2003). Munc 18 preferentially interacts with the closed conformation of syntaxin and might thereby play an inhibitory role in SNARE complex formation (Murthy et al., 2003).

Synaptophysin forms a complex with the vesicular SNARE partner synaptobrevin / VAMP 2 (Becher et al., 1999; Sudhof, 2004) and restricts availability of synaptobrevin / VAMP 2.

Dissassembly of the SNARE complex is regulated by the hexameric ATPase NSF (N-ethylmaleimide-sensitive fusion protein) in conjunction with SNAP proteins (soluble NSF-attachment proteins) (Gundelfinger et al., 2003).

Before fusion occurs a synaptic vesicle must undergo a priming procedure to become fusion competent. Priming follows with some delay after vesicle docking and involves munc 13 and RIMs. Munc 13 interacts with RIM and plasma membrane diacylglycerol (Murthy \& deCamilli, 2003). Deletion of the munc 13 gene in mice results in a complete absence of synaptic vesicle exocytosis (Murthy et al., 2003). RIM is also involved in exocytosis by binding to the small GTPase Rab3 that undergoes cycles of SV association and disassociation in parallel with SV exo- and endocytosis.

SV exocytosis is triggered by an increase in the intracellular calcium concentration upon stimulation. The synaptic vesicle protein synaptotagmin 1 function as the major calcium sensor in exocytosis (Murthy \& deCamilli, 2003; Sudhof, 2004). It harbours two calcium-binding C2-domains that can bind SNARE proteins and plasma membrane phospholipids (Murthy \& deCamilli, 2003). $\mathrm{Ca}^{2+}$-binding might induce interaction of synaptotagmin 1 with plasma membrane proteins and lipids and thereby catalyze SNARE-mediated SV fusion (Murthy \& deCamilli, 2003).

By interacting with the clathrin-adaptor complex AP-2 synaptotagmin 1 also is involved in SV recycling after exocytosis. Synaptic vesicle proteins and excess membrane have to be retrieved from the active zone plasma membrane following exocytosis. Clathrin-mediated endocytosis plays a major role in the recycling of SV components. Alternative vesicles that release their neurotransmitter content through a transient fusion pore may be retrieved by a kiss-and-run mode of endocytosis to replenish the SV pool. Bulk endocytosis is a third way for synaptic vesicle recycling.

During neuronal differentiation clathrin-mediated endocytosis might be involved in the correct sorting of synaptic vesicle proteins and thereby provide a mechanism to establish axonal polarity. 


\subsection{Architecture of the postsynaptic compartment of excitatory synapses}

At the postsynaptic site clathrin-mediated endocytosis of postsynaptic receptors induces changes in the strength of synaptic transmission and is thereby implicated in synaptic plasticity.

In the mammalian brain activity-dependent long lasting changes in synaptic strength are central for processes underlying learning and memory. Longterm depression (LTD) and longterm potentiation (LTP) represent the most thoroughly studied examples of synaptic plasticity in which persistent changes in strength of synaptic transmission are triggered by a short period of synaptic activity. Postsynaptic receptor trafficking in and out of the active zone and the resulting change in abundance of synaptic receptors controls the strength of synaptic signal transmission. A recent study of Rumpel et al. (2005) supports the implication of postsynaptic receptor trafficking for associative learning in living animals. Upon fear conditioning of rats AMPA-type glutamate receptors are incorporated into synapses of postsynaptic neurons in the lateral amygdala. Most of the fast excitatory transmission in the mammalian brain is mediated by glutamatergic synapses. The neurotransmitter glutamate acts on three types of ionotrophic receptors which are named after their selective agonists including NMDA (N-methyl-D-aspartate) receptors, kainate receptors and AMPA ( $\alpha$ amino-3-hydroxy-5-methyl-isoxazole-propionic-acid) receptors. Each receptor comprises a heteromer of different subunits and acts at different stages of induction, expression or modulation of neuronal plasticity. Activation of NMDA receptors induces synaptic plasticity mechanisms such as longterm potentiation (LTP) and longterm depression (LTD). Kainate receptors can trigger forms of LTP or LTD that are independent from NMDA receptor activation.

Excitatory synaptic transmission underlying NMDA induced changes in synaptic plasticity is mediated by AMPA receptors (AMPARs). AMPA receptors are glutamate gated ion channels composed of four subunits that comprise either GluR1 and GluR2 or GluR2 and GluR3. Another subunit,GluR4, is mainly expressed early in development (Zhu et al., 2000). The four subunits differ in the length of their cytoplasmic tails that interact with different sets of proteins (see fig.2.4).

GluR1, GluR4 and a splice variant of GluR2 (GluR2L) have a long cytoplasmic tails whereas GluR2, GluR3 and a GluR4 splice variant GluR4c display shorter tails at the cytoplasmic face of the membrane.

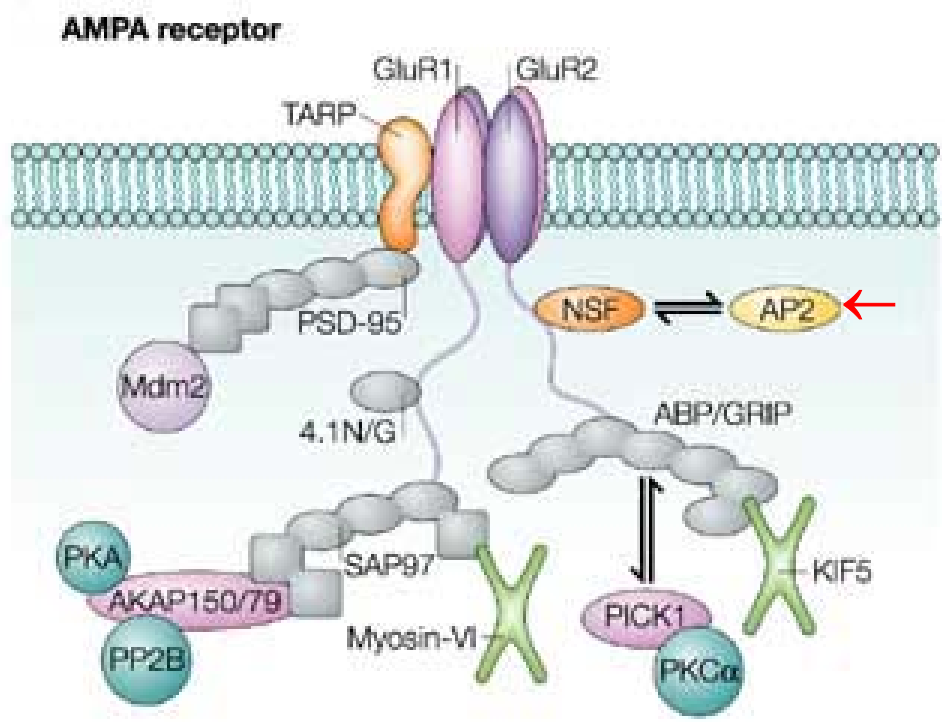

Fig.2.4: The cytoplasmic tail of heterotetrameric AMPA receptors interacts with various proteins at the postynaptic active zone. This work will focusses on binding of AP-2 (see arrow) to a basic motif within the membrane proximal region of the GluR2 cytoplasmic tail that overlaps with a binding site for NSF. Taken from Collingridge et al., 2004 
All four AMPA receptor subunits contain a PDZ binding domain at the very Cterminal portion of their cytoplasmic tails. Subunit specific protein interactions regulate trafficking and synaptic targeting of AMPA receptors. Covalent modification of AMPARs by phosphorylation regulates interaction with specific proteins and thereby affects AMPAR trafficking (Bredt and Nicoll, 2003). As mentioned below AMPA receptor movement in and out of the postsynaptic active zone is highly dynamic during synaptic plasticity.

\subsubsection{AMPA receptor trafficking during longterm potentiation}

LTP upon NMDA receptor activation requires delivery of AMPA receptors to the synapse and activation of synaptic AMPARs by direct phosphorylation. Stimulation of NMDARs in induction of LTP results in an increased dendritic spine calcium concentration (Malenka et al., 1998) and thereby activates the calcium / calmodulin kinase II (CaMKII). CamKII acts on a serine residue at position 831 within the cytoplasmic tail of synaptic GluR1 that is proven to be phosphorylated during LTP (Bredt and Nicoll, 2003). PKA phosphorylation of GluR1 at serine 845 is believed to be necessary for synaptic delivery of AMPARs in LTP (Malinov, 2003). GluR1 is the key subunit which drives synaptic delivery of GluR1 / GluR2 heteromers to the synapse in LTP presumably by interaction between its cytoplasmic tail and the PDZ domain protein SAP97.

Consistent with this overexpression of SAP97 in neuronal cultures increases synaptic AMPA receptor density and the size of spines and leads to an increase in postsynaptic currents (Bredt and Nicoll, 2003).

Stargazin is another PDZ domain protein containing four transmembrane domains. It interacts with all four AMPA receptor subunits and with the postsynaptic density protein PSD 95 that functions as a scaffolding protein at the active zone (Bredt and Nicolls, 2003). Work on mice expressing a mutated stargazin the so called 'stargazer' phenotype demonstrated that stargazin is involved in the recruitment of intracellular AMPARs to the plasma membrane and by interacting with PSD95 it may facilitate synaptic delivery of AMPARs (Bredt and Nicolls, 2003).

In contrast to GluR1 containing AMPA receptors GluR2/GluR3 heteromers cycle continuously between the plasma membrane and intracellular compartments in LTP.

Thus, enhancement of AMPA receptor function during LTP co-incides with facilitated delivery of extrasynaptic or intracellular receptors to the postsynaptic active zone.

This is predominantly mediated by the GluR1 subunit and includes direct covalent modification of synaptic AMPA receptors and activity dependent delivery of AMPA receptors that is regulated by protein interactions with the long GluR1 cytoplasmic tail. 


\subsubsection{AMPA receptor trafficking during longterm depression}

Low frequency stimulation leads to NMDAR induced longterm depression (LTD) the reversal of longterm potentiation. Induction of LTD co-incides with loss of synaptic AMPA receptors (Lee et al., 2002; Beattie et al., 2000; Lin et al., 2000; Man et al., 2000) which are endocytosed at extrasynaptic sites (see fig. 2.5).

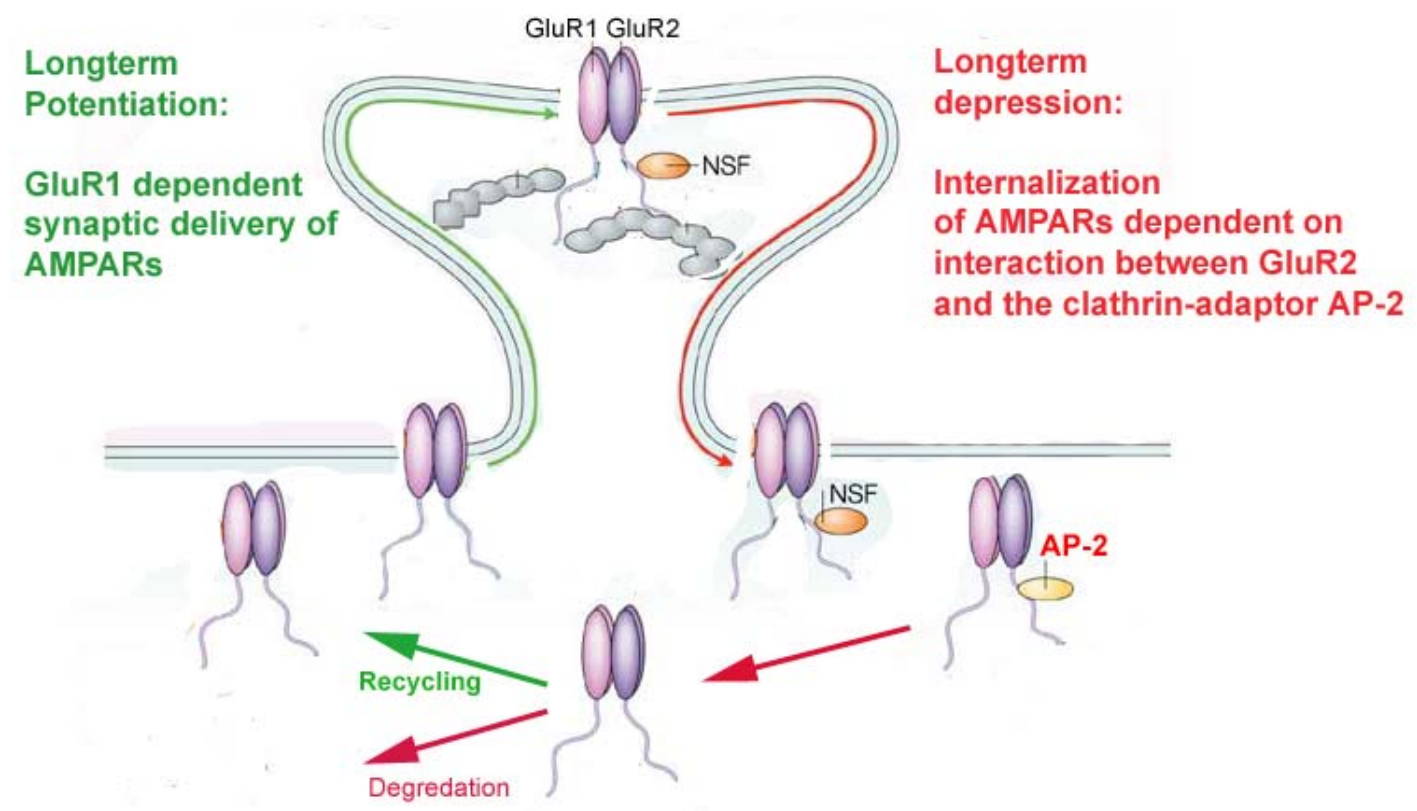

Fig. .2.5: AMPA receptor trafficking during neuronal plasticity. LTD involves the internalization of extrasynaptic AMPARs by clathrin-mediated endocytosis. Recognition of the AMPAR by the clathrin-adaptor complex AP-2 is mediated by a dibasic sorting motif within the AMPA receptor subunit GluR2. Modified from Collingridge et al., 2003.

Endocytosis in dendrites has been shown to occur at specialized endocytic zones adjacent to the postsynaptic density (Blanpied et al., 2002). Thus endocytosis of postsynaptic receptors requires untethering of synaptic receptors and lateral movement to extrasynaptic sites at the plasma membrane. Work of Borgdorff and Choquet (2002) on lateral movement of AMPARs in the plasma membrane has shown the existence of a highly mobile pool of AMPARs besides an immobile pool presumably synaptic receptor pool.

GluR2 is the key subunit that controls NMDA induced internalization of AMPA receptors (Lee et al., 2002; 2005) from the plasma membrane. The short GluR2 cytoplasmic tail interacts with various proteins that regulate trafficking of GluR2 during LTD (see fig. 2.6). 


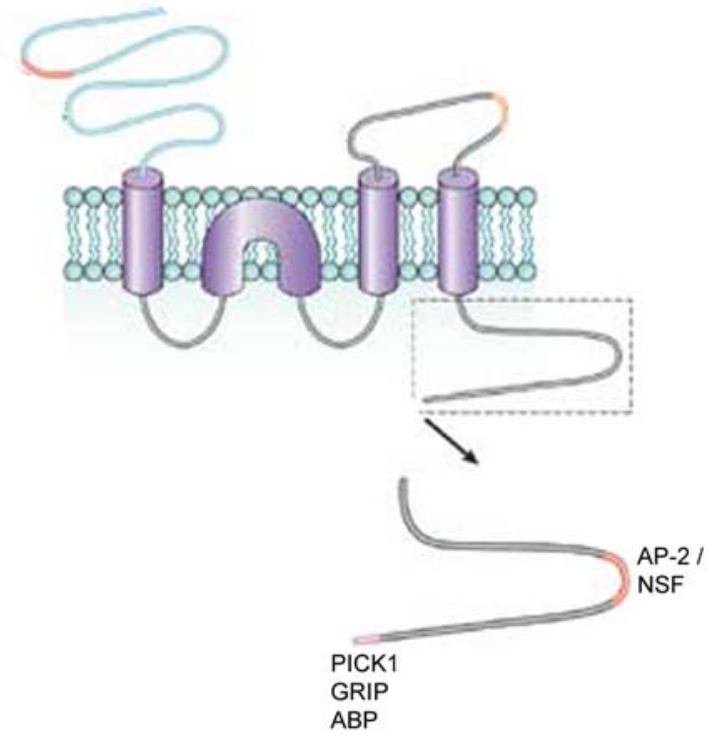

Fig. 2.6: The cytoplasmic tail of the AMPA receptor subunit GluR2 binds to various postsynaptic proteins that are involved in receptor trafficking. The AP2 adaptor complex plays a role in clathrinmediated endocytosis of the receptor and recognizes a basic sorting motif (red residues) in the membrane proximal portion of the cytoplasmic tail that also harbours a binding site for NSF (underlined residues). The very carboxy terminal part of the receptor contains a PDZ binding domain that interacts with the PDZ domain proteins PICK 1 and GRIP dependent on the phosphorylation state of serine 880 (magenta) by protein kinase $C$. Fig. A taken from Collingridge et al., 2004

GluR2 cytoplasmic tail

${ }^{834}$ EFCYKSRAEA $\frac{\text { KRMKVAKNPQ NTNPSSSQNS QNFATYKECY YGIESVKL }}{\text { AP }}{ }^{83} / \mathrm{NSF}$
PICKI $/$
GRIP

The very carboxyterminal portion of GluR2 harbours a PDZ binding domain. Interaction with the PDZ domain proteins ABP/GRIP 1/2 (AMPA receptor binding protein / glutamate receptor interacting protein) and PICK 1 (protein interacting with protein kinase $\mathrm{C}$ ) is regulated by phosphorylation of a serine residue at position 880 by protein kinase $C$. Phosphorylation prevents binding of GRIP 1/2 but enhances interaction with PICK (Collingridge et al., 2004). The membrane proximal part of the GluR2 cytoplasmic tail contains overlapping recognition motifs for the hexameric ATPase NSF and the clathrin-adaptor complex AP-2 (Nishimune et al., 1998; Lee et al., 2002). The binding site for AP-2 is comprised of a dibasic amino acid cluster similar to the AP-2 binding site in the $\mathrm{C} 2 \mathrm{~B}$ domain of the synaptic vesicle protein synaptotagmin 1.

Several lines of evidence demonstrate that clathrin-mediated endocytosis of AMPA receptors from the postsynaptic plasma membrane can be induced by stimulation with agonists such as insulin, AMPA and NMDA (Lin et al, 2000; Man et al, 2000; Beattie et al., 2000). Upon insulin stimulation GluR2 but not GluR1 homomers are internalized from the plasma membrane of transfected HEK cells in a dynamin 1 -dependent manner (Man et al., 2000), suggesting that the GluR2 subunit is the key player in regulated endocytosis of AMPA receptors.

AMPA- or insulin-induced internalization of AMPARs in transfected hippocampal neurons occurs via dynamin 1-dependent mechanisms that differ with regard to their sensitivity towards protein phosphatase inhibitors (Lin et al., 2000).

Blockade of the calcium-dependent-phosphatases calcineurin and protein phosphatase 1 (PPP1) prevents insulin-dependent internalization of AMPARs (Lin et al., 2000) suggesting that in contrast to the requirement of kinase activity for LTP induction of LTD involves phosphatase activity.

Work of Beattie et al. (2000) has shown that activation of NMDARs in LTD is sufficient to induce dynamin-dependent endocytosis of AMPARs from the 
postsynaptic plasma membrane. NMDA causes calcium influx and thereby activates the phosphatase calcineurin which also plays a role in regulation of synaptic vesicle endocytosis at the presynaptic terminal (Bredt and Nicoll, 2003).

NMDA-induced hippocampal LTD requires the interaction between the GluR2 cytoplasmic tail and the clathrin-adaptor complex AP-2 (Lee et al., 2002). Pulldown assays show that binding is dependent on a dibasic motif within the membrane proximal portion of the GluR2 cytoplasmic tail which is overlapping with the recognition site for NSF. Mutations of a lysine and an arginine residue within this motif to alanines interfere with NMDA-stimulated endocytosis of AMPARs in transfected hippocampal neurons but do not affect basal endocytosis of AMPARs. In contrast, deletion of the NSF binding site in the GluR2 cytoplasmic tail from amino acid 849 to 853 does not affect NMDAinduced AMPAR internalization but interferes with AMPA stimulated endocytosis. Deletion of this motif also does not prevent binding of AP-2 from ratbrain extract to the GluR2 cytoplasmic tail (Lee et al., 2002). These results suggest that interaction between the GluR2 cytoplasmic tail and the AP-2 adaptor complex is necessary to mediate clathrin-mediated endocytosis of AMPA receptors during NMDA-induced hippocampal LTD. The presynaptic protein synaptotagmin 1 directly binds to the $\mu 2$-subunit of the AP-2 adaptor complex via a similar dibasic sorting motif (Chapman et al., 1998; Haucke et al., 2000; Grass et al., 2004), suggesting that similar mechanisms may regulate AP2 dependent endocytosis of pre- and postsynaptic membrane proteins.

Basic AP-2 binding motifs are present in the cytoplasmic tails of the AMPA receptor subunits GluR1, GluR2 and GluR3 but only the GluR2 cytoplasmic tail binds to NSF (Lee et al., 2002). This raises the question if differential protein interactions with the AMPA receptor cytoplasmic tails regulate internalization and intracellular targeting of the receptor upon agonist stimulation. Recent work of Lee et al. (2004) demonstrates that the GluR2 cytoplasmic tail fused to the GluR1 subunit stimulates NMDA-induced internalization of the chimera and prevents constitutive endocytosis of GluR1. Thus, the GluR2 cytoplasmic tail is the key player in agonist induced AMPA receptor internalization. Lee et al. (2004) also state that AMPA receptor subunits undergo differential intracellular sorting upon NMDA- or AMPA- induced endocytosis: GluR1 is predominantly targeted to recycling endosomes upon NMDA stimulation whereas GluR2containing AMPARs are sorted for lysosomal degradation which is demonstrated by co-localization with the lysosomal marker LAMP 1. Deletion of the NSF binding site in the GluR2 cytoplasmic tail increases lysosomal targeting of the construct in transfected hippocampal neurons under control conditions and upon AMPA stimulation suggesting that NSF is involved in maintenance of GluR2- containing receptors at the synapse (Lee et al., 2004) These findings are contradictory to previous data of Ehlers et al. (1998) demonstrating that internalized AMPARs are rapidly recycled back to the plasma membrane upon NMDA stimulation.

Other glutamate receptor subtypes such as NMDA or kainate receptors have also been shown to undergo activity-induced exo- and endocytic trafficking (Scott et al., 2004; Lavezzari et al., 2004; Martin et al., 2004) The NMDA receptor is targeted for differential sorting to recycling endosomes or for lysosomal degradation (Scott et al., 2004; Lavezzari et al., 2004). Kainate receptors are sorted for to lysosomes for degradation in a PKC dependent manner upon kainate stimulation. By contrast, NMDA stimulation targets 
receptors to recycling endosomes back to the plasma membrane (Martin et al., 2004). All these data further underline the growing interest in activity-dependent sorting of postsynaptic receptors and its regulation during synaptic plasticity.

In addition to protein interactions covalent modifications including phosphorylation described above and ubiquitination regulate agonist-induced endocytosis of GluR2 receptors.

Interaction with of the GluR2 cytoplasmic tail with the PDZ domain proteins PICK1 and ABP/GRIP1 is modified by protein $C$ kinase phosphorylation. Phosphorylation favours PICK 1 binding to GluR2 and thereby increases internalization of GluR2 containing AMPA receptors from the plasma membrane (Chung et al., 2000; Terashima et al., 2004). Overexpression of PICK 1 in hippocampal slices causes an increase in AMPAR mediated synaptic activity suggesting that interaction with PICK1 is involved in regulation of synaptic strength by changing AMPA receptor subunit composition (Terashima et al., 2004).

Interaction of the GluR2 cytoplasmic tail with PICK1 and NSF has been shown to be involved in calcium-permeable AMPA receptor plasticity (CARP) that regulated the subunit composition of synaptic AMPARs (Gardner et al., 2005). The ATPase activity of NSF is thought to disrupt GluR2 interaction with PICK and thereby facilitates recruitment of AMPARs to the synapse (Gardner et al., 2005, Lee et al. 2002). Findings of Lee et al. (2002) also suggest a role of NSF in maintenance of synaptic and plasma membrane AMPA receptors.

Ubiquitination which targets proteins for degradation, is another type of covalent protein modification that may be involved in regulating the number of synaptic AMPARs during LTD (Colledge et al., 2003; Burbea et al., 2002). Ubiquitination of the C. elegans AMPA receptor GLR1 regulates the abundance of synaptic receptors and thereby affects synaptic strength (Burbea et al., 2002). C. elegans expressing a mutated forms of the clathrin-adaptor protein AP180 / unc-11 internalization of ubiquitinated GLR1 is blocked indicating that clathrinmediated endocytosis of ubiquitinated GLR1 is required for synaptic plasticity (Burbea et al., 2002) The findings of Lee et al., (2004) that endocytosed GluR2 containing AMPARs are targeted to lysosomes for degredation support the view that ubiquitination of postsynaptic receptors may be involved in regulation of endocytosis in synaptic plasticity. Data of Colledge et al. (2003) demonstrate that not the AMPA receptor subunit GluR2 but the postsynaptic density protein PSD 95 is ubiquitinated upon NMDA stimulation of hippocampal neurons by the E3 ligase Mdm2. These results do not exclude a possible ubiquitination of GluR2 because in $\mathrm{C}$. elegans ubiquitination may be more obvisious in comparison to mammals.

PSD 95 is linked to AMPA receptors by the transmembrane PDZ domain protein stargarzin and thereby it functions in tethering of AMPA receptors to the active zone. Degradation of PSD 95 upon ubiquitination releases synaptic AMPA receptors and might thereby facilitate NMDA stimulated AMPAR endocytosis at extrasynaptic sites during LTD.

Thus activity-dependent internalization of AMPA receptors from the postsynaptic plasma membrane during LTD is a tightly regulated process. Regulation includes on the one hand activity dependent phosphorylation of serine or tyrosine residues within the tail and on the other hand involves protein interactions that can also be regulated by phosphorylation. 


\subsection{Synaptogenesis and establishment of neuronal polarity}

Activity dependent synaptic plasticity in the central nervous system requires dynamic changes in the properties of the postsynaptic, as well as of the presynaptic active zone. Activation of postsynaptic NMDA receptors in LTP induces synaptic delivery of AMPARS as mentioned before. In addition, NMDAR activation also triggers structural changes at the presynaptic site that result in formation of new synapses. Thus, synaptogenesis plays an important role during neuronal plasticity. Several studies shed light on synapse formation in mature neurons (see Cohen Cory, 2002; Goda and Davis, 2003). But very little is known about the de-novo formation of synapses during neuronal development.

Neuronal differentiation requires the establishment of axonal and dendritic polarity from an unpolarized precursor cell. The somatodendritic compartment functions as the receptive site for neurotransmitter released from the presynaptic site. Polarity during neuronal differentiation is achieved by differential sorting of proteins such as synaptic vesicle proteins or postsynaptic neurotransmitter receptors to the axonal or dendritic compartment of the developing neuron.

There are several models to study synaptogenesis and the establishment of neuronal polarity. The differentiation of primary hippocampal neurons in culture provides a widely used model to FLAG-Syt1 $\triangle$ C2Bpep2r study developmental processes the in central nervous system (CNS).

Development of primary hippocampal neurons derived from dispersed embryonic day 18 rat brain in culture can be divided into five stages as shown in fig. 2.7 (Dotti et al, 1988): Stage 1 is defined by the formation of motile lamellipodia around the cell periphery shortly after plating. The lamellipodia transform into distinct processes which extend to a length of $10 \mu \mathrm{m}$ to $15 \mu \mathrm{m}$ within a few hours. The outgrowth of these neurites defines stage 2 of the differentiation process. $24 \mathrm{~h}$ after plating one of the neurites starts to elongate very rapidly while the other neurites cease growing. This third stage is characterized by axon formation out of the fastest growing neurite. The cell has become polarized into an axonal and a somatodendritic compartment.

Several lines of evidence indicate that not one of the neurites is predetermined to become the future axon but that each of the identical stage 2 neurites can form the future axon. When one neurite acquires axonal properties the remaining processes are prevented from becoming axons. To achieve polarized sorting of axonal and somatodendritic proteins reorganization of the intracellular sorting machinery from multidirectional to unidirectional transport has to take place. The rapid outgrowth of the axon also requires transport of membrane components and organelles.

Dendrite growth and formation out of the shorter neurites in stage 4 neurons occurs 2 or 3 days after axonal outgrowth. Dendrites elongate more slowly than the axon and unlike the axon several dendrites grow simultaneously.

Mature stage 5 hippocampal neurons are obtained after 7 days in culture. 


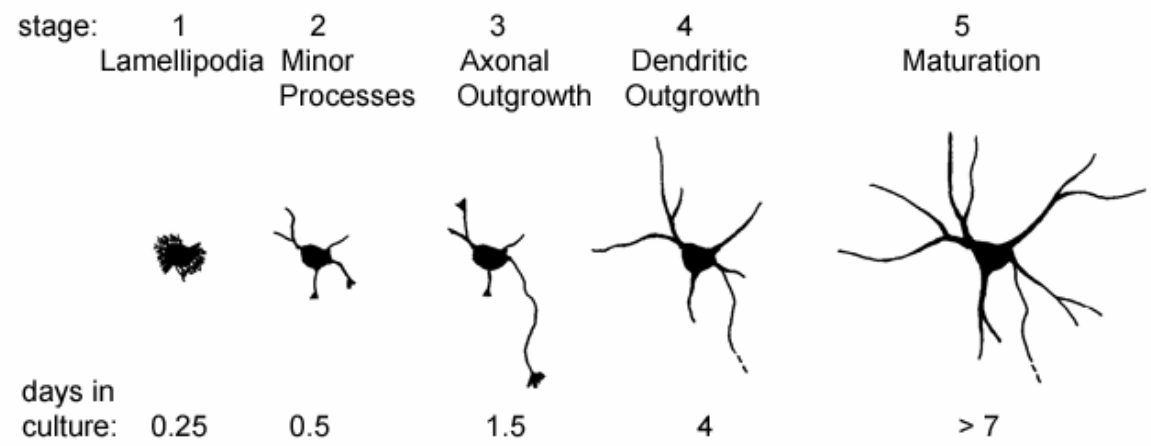

Fig.2.7: Stages of developmentof primary hippocampalneurons in culture. Taken from Dotti et al., 1988

Primary hippocampal neurons have been widely used as a model to study synaptogenesis in the central nervous system.

Synaptogenesis requires the establishment of an asymmetric intercellular junction between an axon and the dendrite of a neighbouring neuron. This process is triggered by reciprocal signalling between the pre- and the postsynaptic compartment. Contact formation between motile dendritic filipodia and the axon represents the onset of synaptogenesis (Cohen-Cory, 2003). Neurotransmitter release from exocytic hotspots of the growing axon regulates filipodial motility (Kraszewski et al., 1995; Dailey \& Smith, 1996; DePaola et al., 2003). Thereby contact formation is facilitated and initial axonal-dendritic contacts become stabilized.

Neurotrophic growth factors including nerve growth factor (NGF) and brain derived neurotrophic factor (BNDF) promote synapse formation in addition to their function in neuronal survival and differentiation (Cohen-Cory, 2003).

Synaptogenesis is modulated by a number of homophilic and heterophilic adhesion molecules that stabilize the axonal-dendritic contact site. $\mathrm{N}$-cadherins and protocadherins mediate calcium-dependent homophilic adhesion (Goda \& Davis, 2003). Postsynaptically localized neuroligins interact with presynaptic neurexins (Missler \& Sudhof, 1998) and trigger the de-novo formation of presynaptic structures (Scheiffele et al., 2000; Cohen-Cory, 2003). Ephrins that bind to Eph tyrosine kinase receptors bind and cluster postsynaptic NMDA receptors and thereby promote synapse assembly (Goda \& Davis, 2003).

The third step in synaptogenesis is represented by the assembly of active zone elements at the pre- and postsynaptic sites. In this process presynaptic active zone components are assembled prior to postsynaptic specialization. Synapse formation occurs within 1 or 2 hours after initial contact formation and proceeds very rapidly (Friedman et al., 2000; Goda \& Davis, 2003). Out of this finding the question arose how the rapid assembly of active zone components is achieved during synaptogenesis. Several lines of evidence imply that active zone components are transported in pre-fabricated packets to the nascent synapse. Work of Zhai et al. (2001) characterizes an active zone precursor vesicle that includes the active zone proteins and components of the exocytic machinery. These components are transported to the future active zone prior to the appearance of electron lucent SVs on $80 \mathrm{~nm}$ dense core granulated vesicles that are reminiscent to neuropeptide containing large dense core vesicles (Zhai et al., 2001). Fusion of the active zone precursor vesicle with the presynaptic plasma membrane might be involved in formation of the active zone. The active 
zone precursor vesicle is devoid of synaptic vesicle proteins such as synaptophysin or synaptobrevin / VAMP 2. Synaptobrevin 2 is transported along the growing axon together with the synaptic vesicle proteins SV 2 and synapsin 1 and the endocytic accessory protein amphiphysin in discrete packets (Ahmari et al., 2000). These transport packets have a heterogeneous morphology including dense core vesicles, pleimorphic small vesicles and tubovesiclar structures but no synaptic vesicle like structures (Ahmari et al., 2000).

Assembly of the postsynaptic active zone occurs after presynaptic specialization. It seems that formation of the presynaptic active zone triggers the assembly of postsynaptic active zone components by reciprocal signalling (Goda \& Davis, 2003). Postsynaptic specialization is first characterized by the appearance of NMDA receptors and the postsynaptic scaffolding protein PSD 95 followed by accumulation of AMPA receptors (McGee \& Bredt, 2003; Friedman et al., 2000).

Postsynaptic active zone assembly may in analogy to presynaptic specialization involve delivery of active zone components in prefabricated transport packets as well as the de-novo clustering of postsynaptic proteins at the synapse (Goda \& Davis, 2003). These observations imply the questions how synaptic vesicles are formed from a precursor organelle during neuronal differentiation.

Besides inter-neuronal communication neuron-glia signalling is involved in synapse formation. Glia-derived cholesterole promotes synaptogenesis in cultured hippocampal neurons (Mauch et al., 2001). Cholesterol is also a component of detergent insoluble complexes that are implied in the correct sorting of axonal or dendritic proteins in establishment of neuronal polarity (Ledesma et al., 1999; Hering et al., 2003).

\subsubsection{Cell culture models for neuronal differentiation}

As mentioned before primary hippocampal neurons are a widespread model to study neuronal development in cell culture (Dotti et al., 1988). Autaptic cultures of a single neuron on a micro-glia island provide a valuable tool to study synaptogenesis. But one major drawback of primary neurons to study synaptic vesicle biogenesis is the expression of synaptic vesicle proteins from earliest stages on.

Thus, in-vitro differentiation of unpolarized embryonic precursor cells into neurons provides an appropriate alternative model to study synaptic vesicle biogenesis. Pluripotent embryonic stem cells and embryocarcinoma cells can be in-vitro differentiated into postmitotic neuronlike cells by retinoic acid induction. The murine embryo carcinoma cell line P19 was isolated by implantation of a 7-day-old mouse embryo under the testis capsule of an adult which resulted in tumor formation (McBurney et al., 1972). Tumor cells were expanded in cell culture and the clone P19 was established as a cell line.

P19 cells are similar to cells of the primary ectoderm; they proliferate rapidly in cell culture and express several early embryonic markers i. e. oct3.

P19 cells can differentiate along a neuronal, cardiac or skeletal muscle pathway in culture. For neuronal differentiation P19 precursor cells are cultured as aggregates under exposure to retinoic acid for a few days (fig. 2.8). Culturing the cells as aggregates facilitates embryonic body formation and juxtacrine signalling that plays an important role in differentiation. After dissociation of the 
aggregates and plating on an adhesive substrate the cells differentiate into a population of postmitotic neuron-like and glia-like cells.

P19 neuronlike cells are segregated into an axonal and a somatodendritic compartment (Finley et al. 1995). They express a variety of neuronal markers such as synaptic vesicle proteins, glutamic acid carboxylase, GABA receptors, which form part of inhibitory synapses (Bain et al., 1993), NMDA, kainate and AMPA receptors which form part of excitatory synapses (Turetsky et al., 1994). P19 neuronlike cells form morphologically (McBurney et al. 1988) and physiologically (Finley et al., 1995) intact synapses which express strong inward and outward currents upon electrical excitation.

Gene expression during neuronal differentiation of P19 cells appears roughly in the same temporal order as in normal neural precursor cells in-vivo (Bain et al., 1994).

The ability of P19 cells to differentiate into postmitotic physiologically functionating neurons along a pathway which strikingly resembles that of normal neuronal precursors in-vivo makes them an adequate in-vitro model to study neuronal differentiation. The following work analyzes expression patterns and intracellular distribution of synaptic vesicle proteins during differentiation of P19 precursors into postmitotic neurons.

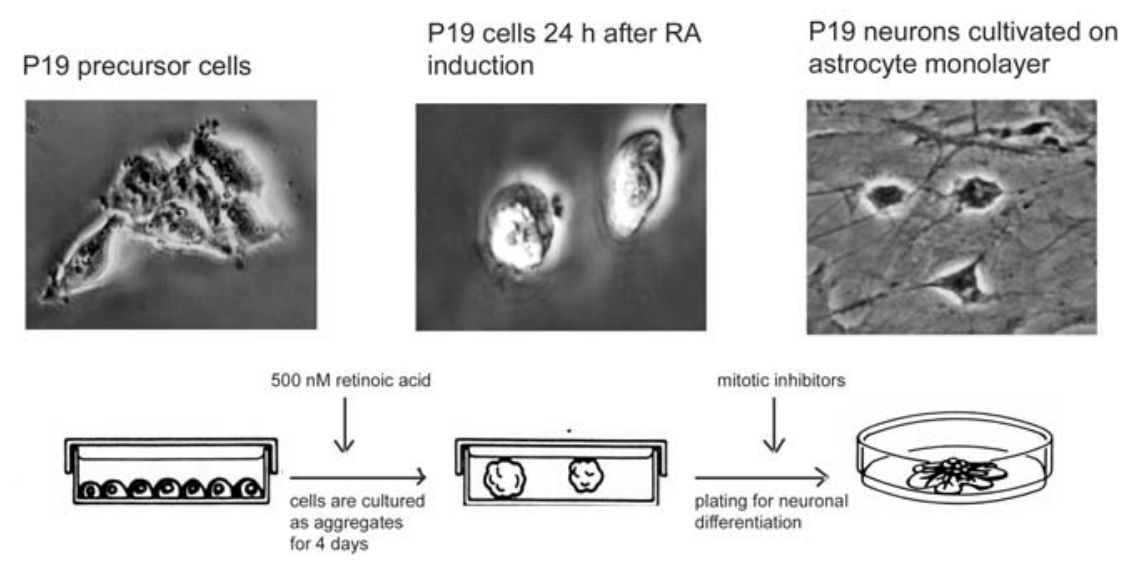

Fig. 2.8: Pluripotent embryocarcinoma cells can be differentiated in-vitro into postmitotic neurons upon retinoic acid induction. Aggregates of P19 precursor cells were exposed to retinoic acid for four days to induce neuronal differentiation. After dissociation of the aggregates neuronal precursor cells differentiate into postmitotic stage 5 neurons within a few days that can be cultured on an astrocyte monolayer.

Apart from the murine embryocarcinoma cell line P19 pluripotent embryonic stem cells and human teratocarcinoma cells can undergo retinoic acid induced neuronal differentiation when cultured as aggregates. Neuronal crest stem cells or neuroblastoma cell lines represent additional models to study processes involved in neuronal differentiation. Many hints on neuronal secretion and formation of synaptic vesicles were gained by studies on neuroendocrine cell lines such as rat pheochromocytoma cells (PC12) (Cameron et al., 1991).

Differentiation of embryonic stem cells into postmitotic neurons by stromal cell induced activity represents an alternative differentiation protocol that is not dependent on chemical induction and results in a higher number of postmitotic neuron-like cells (Kawasaki et al., 2000). For this protocol embryonic stem cells are cultured on a layer of bone marrow derived stromal cells that induces neuronal differentiation by secretion of up to now unknown factors. The majority 
of ES cells differentiate into dopaminergic neurons. Therefore this differentiation protocol is widely used in research on Parkinson's disease.

Taken together, in-vitro differentiation of embryonic precursor cells into postmitotic neurons provides a valuable tool to study very early stages during neuronal development.

\subsubsection{Expression and sorting of synaptic vesicle proteins during neuronal differentiation}

Neuronal differentiation requires the synthesis of synaptic vesicle associated proteins and their correct sorting to the axonal compartment. In fibroblasts cotransfected with synaptotagmin 1, synaptophysin and SV 2 it could be shown that synaptic vesicle proteins are sorted independently to distinct cellular compartments (Feany et al., 1993). Synaptotagmin 1 is targeted to the plasma membrane, whereas synaptophysin and SV 2 are sorted to small intracellular vesicles in which they do not co-localize. Synaptophysin containing microvesicles were positive for endocytosed transferrin while the SV2 containing vesicles were not. Thus synaptic vesicle proteins that are targeted to the same organelle in polarized neurons are sorted independently to different organelles in unpolarized fibroblasts.

Synaptophysin exclusively localizes to an endosomal transferrin receptor positive compartment in transfected fibroblasts. Neuroendocrine cells in contrast contain two pools of synaptophysin as could be shown by glycerole velocity gradient fractionation of low speed supernatants (Cameron et al., 1991). One peak in which synaptophysin co-migrates with the transferrin receptor seems to be of endosomal origin whereas another peak is devoid of transferrin receptor. These results imply the hypothesis that the endosomal pathway which recycles synaptophysin together with the transferrin receptor from the plasma membrane is involved in the formation of synaptic vesicle like microvesicles (SLMVs) in neuroendocrine cells.

Sorting of synaptic vesicle proteins to distinct organelles in unpolarized cells and the finding of a non-endosomal organelle containing synaptophysin in neuroendocrine cells gives rise to the question if the endosomal recycling pathway plays a role in the formation of synaptic vesicles. It is also unclear how polarized sorting of synaptic vesicle proteins is achieved during differentiation of an unpolarized precursor cell into a neuron.

Expression of synaptic vesicle proteins occurs before neurite outgrowth and synaptogenesis (Fletcher et al., 1994). Synaptotagmin 1, synaptobrevin 2 and synapsin 1 proteins are expressed at constant levels but their half-life increases during differentiation of primary hippocampal neurons (Fletcher et al., 1994). Stability of these proteins increases in parallel with the increase of the synaptic vesicle pool leading to the assumption that incorporation of proteins into synaptic vesicles elongates their half-life. In contrast to synaptotagmin 1, synaptobrevin 2 and synapsin 1 synthesis of synaptophysin is dramatically increased during neuronal differentiation (Fletcher et al., 1994). This might enable synaptophysin to play a role in synaptic vesicle formation during neuronal differentiation. The existence of the non-endosomal synaptophysin containing organelle in neuroendocrine cells also supports this idea. 
In adult rat brain synaptophysin forms a stable complex with the SNARE protein synaptobrevin 2. This complex is not detectable in embryonic rat brain; its formation is up-regulated during neuronal differentiation (Becher et al., 1999).

Taken together these findings imply a role for the endocytic recycling of synaptic vesicle proteins from the plasma membrane in the maturation of synaptic vesicles during neuronal differentiation.

Axonal and dendritic outgrowth requires de-novo synthesis of membrane lipids. A study of Ledesma et al. (1999) revealed that biosynthesis of sphingomyelin is up- regulated during neuronal differentiation of primary hippocampal neurons. Sphingomyelin forms part of detergent-insoluble complexes (DICs) which function in the correct sorting of axonal proteins. The specific axonal localization of Thy-1 correlated with an increase in sphingomyelin concentration during neuronal differentiation.

In contrast, biosynthesis of cholesterol another DIC component is not upregulated in neuronal differentiation. Cholesterole required for neuronal maturation and synaptogenesis in retinal ganglion cells is provided by glia cells (Mauch et al., 2001). The synaptic vesicle protein synaptophysin interacts with cholesterole on the membrane of SLMVs in PC12 neuroendocrine cells (Thiele et al., 2000). Cholesterole depletion in these cells does not affect endocytosis but blocks SLMV biogenesis. Thiele et al. suggest that association of cholesterole with synaptophysin oligomers might promote membrane curvature and SLMV biogenesis.

Interaction between synaptic vesicle proteins and DIC components as well as up- regulation of sphingomyelin biosynthesis and the promoting activity of cholesterole on synaptogenesis seem be crucial for synaptic vesicle biogenesis during neuronal differentiation.

In primary hippocampal neurons GFP-synaptobrevin 2 is transported along the developping axon together with other components of the presynaptic active zone in discrete packets (Ahmari et al., 2000). These packets do not have the uniform shape and size of $50 \mathrm{~nm}$ synaptic vesicles. Instead, they contain a very heterogeneous composition of dense-core vesicles, tubovesicular structures and pleiomorphic vesicles. This observation suggests that substantial membrane remodelling is necessary to generate uniformly shaped synaptic vesicles at the active zone and that clathrin-mediated recycling of synaptic vesicle proteins may play an important role in synaptic vesicle maturation.

\subsection{Clathrin-mediated endocytosis at the synapse}

Clathrin-mediated endocytosis (CME) is a mechanism to internalize transmembrane proteins from the cellular surface by means of a clathrincoated-vesicle (CCV) which is then transported to the endosomal system where endocytosed proteins are sorted either for lysosomal degradation or recycling to the plasma membrane. CME has an important implication in nutrient uptake, cellular signalling, cellular homoeostasis and cell to cell interactions. In the nervous system CME is involved in regulated uptake of proteins from both sides of the synapse. At the presynaptic terminal synaptic vesicle proteins and excess plasma membrane from exocytosed synaptic vesicles (SV) are retrieved by $\mathrm{CME}$, and the number of postsynaptic neurotransmitter receptors in neuronal plasticity is also regulated by clathrin-mediated internalization. 
A clathrin-coated bud or vesicle is comprised of three layers. The outer sheath contains cargo proteins and plasma membrane proteins. A clathrin-adaptor complex AP-2 is recruited to the plasma membrane by binding to endocytic sorting motifs in the cytoplasmic tail of cargo proteins and interaction with plasma membrane phospholipids. The AP-2 complex functions as an intermediate layer which recruits clathrin and accessory proteins that aid and regulate vesicle formation and thereby links transmembrane cargo to the outer clathrin-coat (see fig. 2.9).

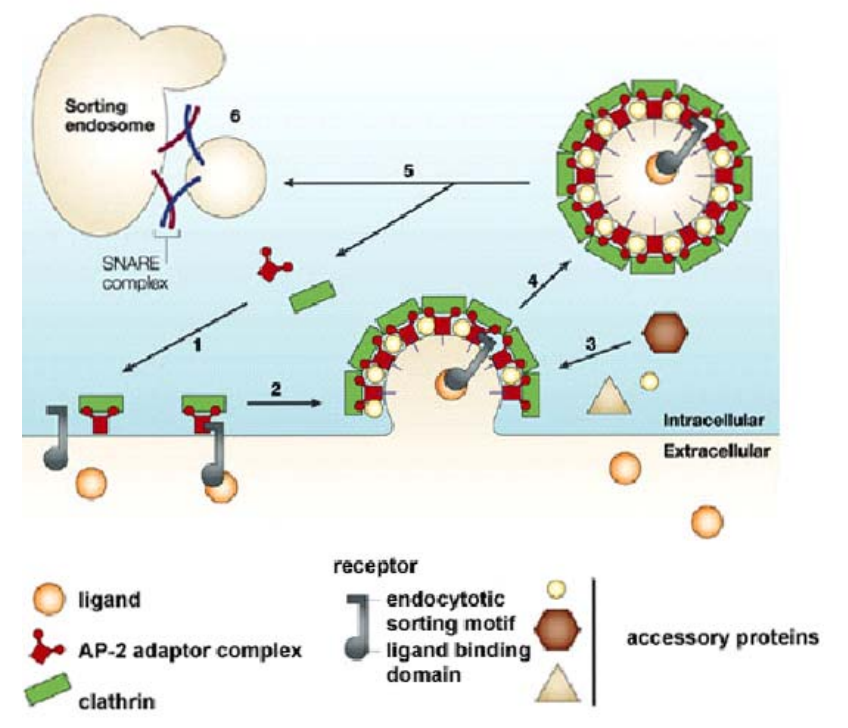

Fig. 2.9: Clathrin-mediated endocytosis of a transmembrane receptor. Upon ligand binding the activated transmembrane receptor recruits the clathrin-adaptor complex AP2 which recognizes a short endocytic sorting motif in the receptor cytoplasmic tail and binds to phospholipids of the plasma membrane. AP-2 then recruits the clathrin-coat and accessory proteins that aid in vesicle formation. A clathrincoated vesicle buds of the plasma membrane and is transported to the endosomal system. Taken from Maxfield \& McGraw, 2004

Nature Reviews | Molecular Coll Biology

CCVs function in trafficking between from the plasma membrane to the endosomal system and in transport between the trans-Golgi network (TGN) and endosomes. CCVs which allowed identification of clathrin as coat protein were first isolated by Pearse et al. (1975). Clathrin the major organiser of the coat is a triskelion of three extended subunits each comprising a heavy and a light chain that radiate from a central hub (Ungewickell, 1983; Kirchhausen et al., 1983). The $N$-terminal domain of clathrin has a $\beta$-propeller structure which allows multiple protein interactions by binding to certain peptide motifs (Owen et al. 2004). The triskelia polymerize into a coat around a vesicle forming a lattice of open hexagonal and pentagonal faces (Fotin et al. 2004; reviewed in McMahon and Mills 2004). Clathrin-coated structures vary in curvature and size facilitating the adjustment of coated pit size to the diameter of cargo (Ehrlich et al., 2004). A variety of accessory proteins aids in clathrin-recruitment, membrane bending and fission of a CCV from the plasma membrane. AP180, epsin and huntingtin interacting protein (HIP1 / HIP1R) are involved in cargo recruitment and clathrin-coat polymerization (McMahon and Mills 2004). Amphiphysin, endophilin and epsin function as inducers or sensors of membrane curvature (McMahon and Mills 2004). Amphiphysin recruits the GTPase dynamin which oligomerizes to form a ring around the neck of a clathrin-coated invagination. Upon GTPhydrolysis dynamin 1 pinches of the clathrin-coated pit from the plasma membrane which gives rise to a clathrin coated vesicle. A defect in 
synaptic vesicle recycling in the fly mutant 'shibire' is due to a defect in dynamin 1 mediated scission of SVs above a permissive temperature.

The CCV is rapidly uncoated in the cell by help of accessory proteins such as auxillin and the phosphatase synaptojanin (McMahon and Mills, 2004). The uncoated vesicle then undergoes fusion with an early endosome and releases its cargo for further trafficking through the endosomal pathway. An internalized transmembrane protein can be targeted for lysosomal degradation or be recycled to the plasma membrane via a late recycling endosome. Endosomal or lysosomal sorting signals in transmembrane proteins consist in general of a short linear array of four to seven amino acids which is recognized by proteins of the endocytic machinery such as clathrin and the AP-2 adaptor complex (Bonifacino and Traub, 2003). Alternatively covalent modification of cargo proteins such as postsynaptic AMPA receptors by phosphorylation or ubiquitination serves as a recognition signal for endocytosis and intracellular trafficking (Bonifacino and Traub, 2003).

At the nerve terminal CME has an implication in the regulated uptake of proteins from both sides of the synapse.

At the presynaptic terminal synaptic vesicles fuse with the active zone plasma membrane to release neurotransmitter into the synaptic cleft (fig. 2.10). After exocytosis excess membrane and synaptic vesicle proteins have to be recycled from the plasma membrane. Three different modes of endocytosis implicated in SV recycling have been described up to now (Royle and Lagnado, 2003). An implication of CME in synaptic vesicle recycling at the frog neuromuscular junction was first described by work of Heuser and Reese (1973). Kiss-and-run endocytosis in which the synaptic vesicle releases its neurotransmitter content through a transient fusion pore and does not collapse into the plasma membrane was proposed to exist by work of Valtorta et al. (2001). A third way for retrieval of SV proteins and membrane is bulk endocytosis (Royle and Lagnado, 2003).

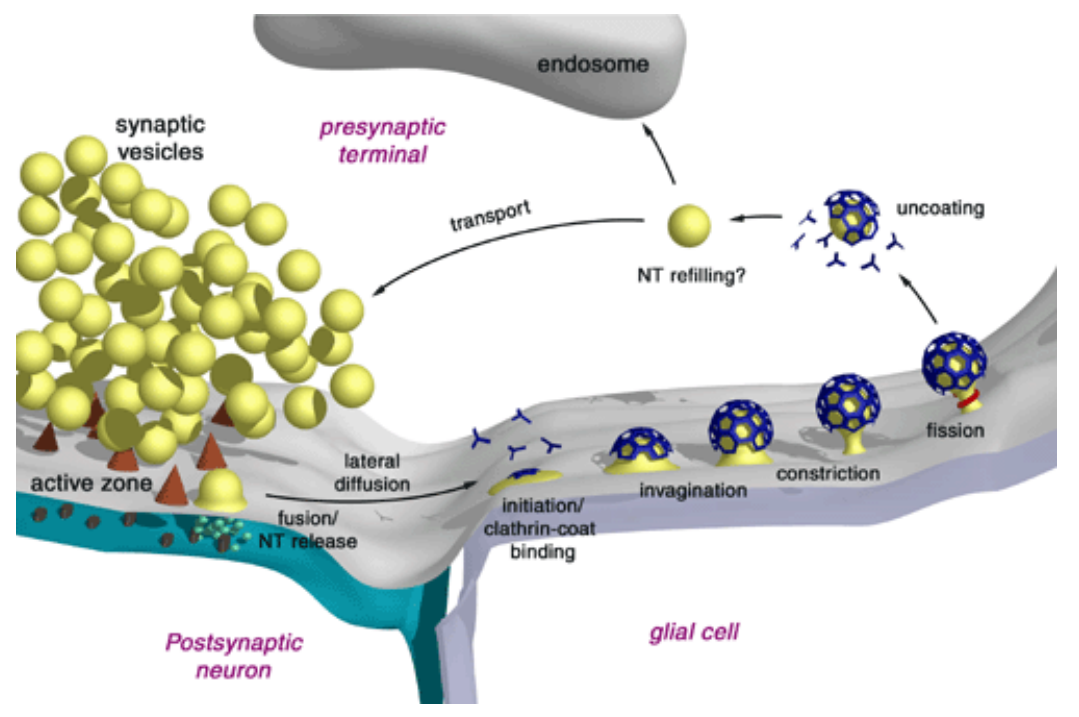

Fig.2.10: Synaptic vesicle recycling at the presynaptic nerve terminal. After exocytosis of synaptic vesicle proteins and membrane components are retrieved by clathrin-mediated endocytosis. The internalized synaptic vesicle can be refilled with neurotransmitter or undergo fusion with an early endosome. Taken from Helge Gad, Karolinska institutet, Sweden 
At the postsynaptic side CME of neurotransmitter receptors plays an important role in synaptic plasticity. In NMDA induced hippocampal longterm depression AMPA receptors are internalized from the plasma membrane in an AP-2 dependent manner (Lee et al. 2002). Constitutive internalization of inhibitory GABA $_{A}$ involves dynamin1 and AP-2 (Kittler et al., 2000; Herring et al., 2003). NMDA receptors also locate to the endosomal system after internalization from the postsynaptic plasma membrane (Laverazzi et al., 2004, Scott et al, 2004).

This work will focus on a detailed study of interaction between the AP-2 complex and the postsynaptic AMPA receptor via a basic recognition motif in clathrin-mediated endocytosis. Another part of the work analyzes a putative recycling compartment for synaptic vesicle proteins in neuronal precursor cells which might play a role in synaptic vesicle biogenesis during neuronal differentiation.

\subsubsection{Recognition of cargo proteins during clathrin-mediated endocytosis}

As mentioned before adaptor proteins serve as a link between transmembrane cargo proteins and the clathrin-coat of a vesicle. These adaptor proteins include the classical heterotetrameric complexes AP-1 and AP-2, and the monomeric GGA proteins (Golgi-associated, $\gamma$-adaptin homology ADP-ribosylation factor (ARF) binding proteins) (McMahon and Mills, 2004). Other monomeric adaptors include AP180 / CALM, which is involved in regulation of the vesicle size (Owen et al., 2004), ARH, Dab2, $\beta$-arrestins, stonins and epsins that induce membrane curvature and promote clathrin-polymerization (McMahon and Mills, 2004). All clathrin-adaptors share a common design of discretely folded domains, which are joined by an unstructured flexible linker that allows them to simultaneously bind to plasma membrane components on the one hand and to clathrin, on the other hand.

The AP-2 complex is a key player in clathrin-mediated endocytosis of proteins from the plasma membrane. Together with AP-1, AP-3 and AP-4 the plasma membrane adaptor AP-2 belongs to the family of heterotetrameric clathrin adaptor complexes acting at different stations of intracellular trafficking. AP-1 is involved in protein sorting between the TGN and endosomes. Lysosomal sorting of cargo is mediated by AP-3. AP-4 is found on vesicles in the vicinity of the TGN (McMahon and Mills, 2004).

Each of these adaptor complexes comprises two large subunits ( $\alpha$ and $\beta 2$ in AP-2), a medium ( $\mu 2$ in AP-2) and a small chain ( $\sigma 2$ in AP-2) (see fig.2.11). $\alpha-$ and $\beta 2$-adaptin each have a size of 110 to $130 \mathrm{kDa}$. They can be subdivided into three domains: an N-terminal trunk domain of 60 to $70 \mathrm{kDa}$ which is part of the AP-2 core complex a hinge domain, and a carboxy-terminal appendage or ear domain of 15 to $30 \mathrm{kDa}$ (Owen et al., 2003). The $\mu 2$ (50 kDa) and $\sigma 2$ (20 $\mathrm{kDa}$ ) subunits form part of the AP-2 core complex. The crystal structure of the AP-2 core complex was solved by David Owen and collegues (Collins et al., 2002). The core is a highly compact rectangle. The $\alpha$ - and $\beta 2$-trunk domains form its outside. The medium $\mu 2$ and small $\sigma 2$-subunit nestle in the elbows of the $\alpha$ - and $\beta 2$-subunits (Collins et al., 2002). Cargo binding of the AP-2 adaptor complex is mainly mediated by its $\alpha$ - and $\mu 2$-subunits. 


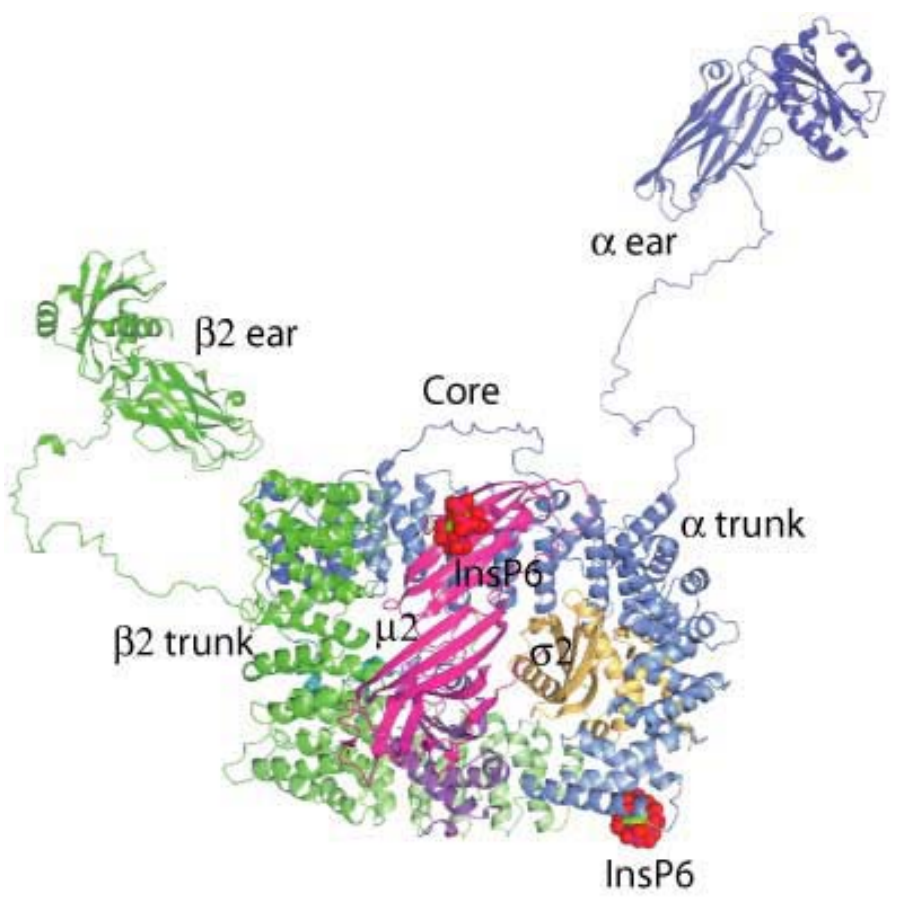

Fig. 2.11: Structural model of the AP-2 complex. The heterotetrameric complex comprises of two large subunits $\alpha$-(blue) and $\beta 2$-adaptin (green) which can be subdivided into an $\mathrm{N}$-terminal trunk and a C-terminal ear- or appendage domain a medium subunit $\mu 2$ (magenta) and a small subunit $\sigma 2$ (yellow) which form the core complex together with the $\alpha$-and $\beta 2$-trunks. (Collins et al. 2002)

As mentioned short linear arrays of amino acids function as recognition motifs for binding of adaptors to transmembrane cargo proteins and to clathrin. Clathrin-binding by AP-2 occurs by a so-termed clathrin box motif which comprises the sequence $\mathrm{L} \phi x \phi[D E]$ where $\phi$ is a bulky hydrophobic residue (Dell' Angelica et al., 1998). The motif binds to the clathrin amino-terminal propeller domain (ter Haar, et al., 2000; Owen et al., 2004). A LLNLD motif mediates clathrin-binding of the $\beta 2$ hinge domain (McMahon and Mills, 2004).

AP-2 is recruited to the plasma membrane by direct binding of phosphatidylinositol $(4,5)$-bisphosphate to the $\alpha$-adaptin trunk domain and a cluster of lysines in the $\mu 2$-subunit (Owen et al. 2004, Rohde et al., 2002). Phosphatidylinositol (4,5)-bisphosphate $\left[(P t d I n s)(4,5) \mathrm{P}_{2}\right]$ and perhaps (Ptdlns)(3,4,5) $\mathrm{P}_{3}$ serve as plasma membrane markers. In contrast, the Golgi membrane marker (PtdIns)(4)P functions together with the small G-protein Arf1 in recruitment of AP-1 to the Golgi membrane (Owen et al. 2004).

In many if not all cases adaptor recruitment to membranes is aided by activated small G-proteins from the Arf / Arl / Sar family (Owen et al., 2004).

Transmembrane cargo proteins interact with the AP-2 adaptor complex by short linear amino acid sequences termed sorting motifs in their cytoplasmic portion. Several types of sorting motifs recognized by AP-2 including tyrosine-based sorting motifs, dileucine-based motifs (DE) $x \times x L L(I)$, in which an acidic resiue precedes a cluster of two leucines or a leucine and an isoleucine, and basic sorting motifs have been described up to now. Tyrosine-based sorting motifs consist of a NPXY or YXXФ consensus motif, where $\mathrm{x}$ represents a variable residue and $\Phi$ represents a bulky hydrophobic amino acid (Owen et al. 2004). 
The YXXФ sorting motif is widely used in protein sorting and is present in the cytoplasmic portion of several endocytic receptors such as the transferrin, the epidermal growth factor (EGF) and the asialoglycoprotein receptor. Tyrosinebased sorting motifs are generally recognized by $\mu 2$-adaptin. Most knowledge upon this interaction motif comes from work of Owen et al. (1998) in which $\mu 2$ was crystallized in presence of a tyrosine-based synthetic peptide. Thus, the binding site for tyrosine-based sorting motifs was identified.

The first 157 amino acids of $\mu 2$ are predominantly organized in an $\alpha$-helical structure and link the subunit to the AP- 2 core by interaction with $\beta$-adaptin. The C-terminal portion of $\mu 2$ from amino acid 158 to 435 is comprised of $\beta$-sheets which form a banana shaped structure and can be divided into two subdomains A (aa 158 to 282 and aa 395 to 435) and B (282 to 394) (Owen et al., 1998, Collins et al., 2002). The tyrosine based sorting motif $Y X X \Phi$ binds via hydrophobic interaction of tyrosine and $\Phi$ to a compatible pocket in the $\mu 2$ subdomain A. Crystallization studies on the AP-2 core complex comprising the trunk domains of $\alpha$-and $\beta 2$-adaptin, $\mu 2$ and $\sigma 2$ revealed that the recognition site for tyrosine based sorting motifs in $\mu 2$-adaptin is blocked by the $\beta$-subunit (Collins et al. 2002) in a way that AP-2 is not capable of binding to YXXФ in this 'closed' conformation. The in-vivo existence of this conformation would require the induction of a conformational change to an open state to allow binding of endocytic signals. Phosphorylation of AP-2 might serve to induce this change in conformation: AP-2 has been shown to be phosphorylated on the $\alpha-$, $\beta 2-$ adaptin and $\mu 2-$ subunit (Owen et al., 2004). The protein kinase AAK 1 phosphorylates $\mu 2$ at position 156 at the beginning of subdomain A (Owen et al. 2004). Phospho- $\mu 2$ shows an increased binding affinity for $Y X X \Phi$ and acidic dileucine signals (Ricotta et al., 2002, Fingerhut et al. 2001). In the closed conformation the phospholipid binding sites in $\alpha$ - and $\mu 2$-adaptin cannot bind simultaneously to the plasma membrane because they are located on perpendicular faces of the complex (Collins et al., 2002). This finding suggests a model by which phosphorylation of the $\mu 2$ subunit by AAK 1 triggers a conformational change within AP-2 from a closed to an open state causing the $\mu 2 \mathrm{C}$-terminal portion to flip out. This putative conformational activation would free the binding pocket for tyrosine- based sorting motifs and allow simultaneous binding of $\mu 2$ and $\alpha$-adaptin to plasma membrane phosphatidylinositol (4,5)-bisphosphate (see fig. 2.12). 


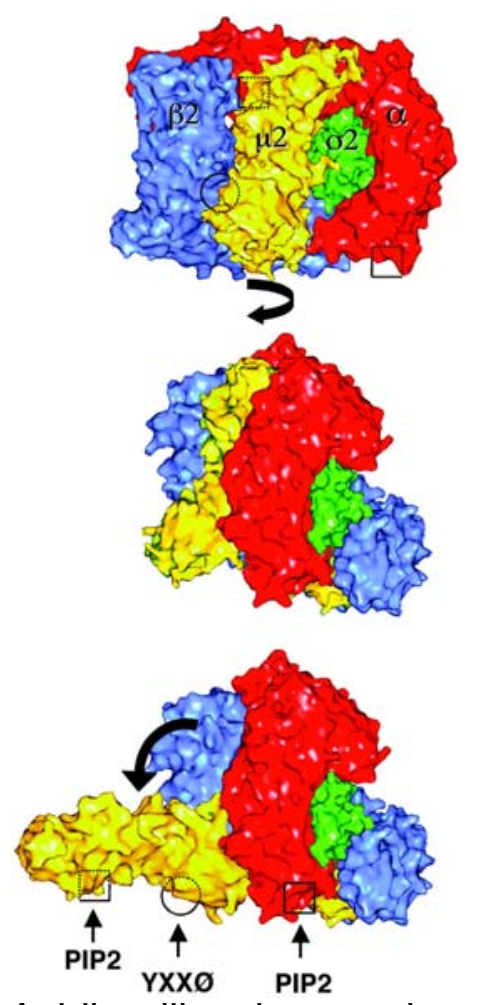

Fig. 2.12: Structural model for a conformational change in the AP-2 complex, which allows recognition of a tyrosine, based sorting motif $Y X X \Phi$ at the plasma membrane. Upon phosphorylation of $\mu 2$ by AAK 1 at position $156 \mu 2$-adaptin is caused to flip out of the AP-2 core complex. The change from a closed to an open conformation enables $\alpha$ and $\mu 2$-adaptin to simultaneously bind to plasma membrane phospholipids and gives access to

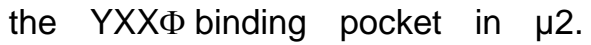
Taken from Bonifacino and Traub, 2003.

Acidic dileucine sorting motifs are present in a variety of transmembrane receptors such as the T-cell antigen receptor CD3 or the co-receptor CD4 (Bonifacino and Traub, 2003). The recognition site for dileucine based sorting signals in the AP-2 adaptor complex is distinct from the binding pocket for tyrosine based sorting motifs. Interaction of dileucine signals with $\mu 2, \mu 1$ and $\mu 3 \mathrm{~A}$ has been described and there is also evidence for interaction with the $\beta 2$ trunk (Bonifacino and Traub, 2003), but the precise mode of interaction with AP2 remains yet to be determined. Work on epidermal growth factor receptor internalization by Huang et al. (2003) shows an implication of $\beta 2$ phosphorylation on clathrin-mediated endocytosis of the receptor.

Tryptophane based sorting motifs such as WXXF or WVXF represent another class of endocytic sorting motifs. Their interaction with the $\alpha$-adaptin ear domain and $\mu 2$ has been recently described for various proteins such as the neonatal Fc receptor (Wernick et al., 2005). Basic clusters in the cytoplasmic portion of transmembrane cargo proteins can also serve as recognition signals for AP-2 in clathrin-mediated endocytosis. Such a motif has been first described by work of Aroeti et al. (1994) on basolateral recycling of the polymeric immunoglobulin receptor.

Basic sorting motifs in the cytoplasmic tails of various proteins from the pre- and postsynaptic site have been shown to mediate interaction with the AP-2 adaptor complex (see Fig.2.13).

\section{Synaptotagmin 1 \\ AMPA receptor GluR2 \\ GABA receptor $\beta 3$ \\ Polmeric immunoglobulin receptor}

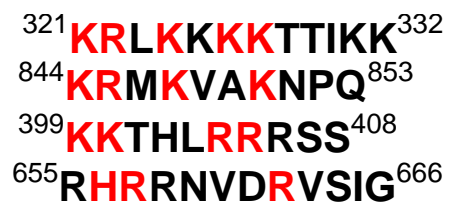

Fig. 2.13: Sequence alignment of basic clusters in the cytoplasmic tails of synaptotagmin 1, the AMPA receptor subunit GluR2, the $\mathrm{GABA}_{A}$ receptor subunit $\beta 3$ and the polymeric immunoglobulin receptor. 
The synaptic vesicle protein synaptotagmin 1 harbours a basic cluster within its C2B-domain that is recognized by the $\mu 2$-subunit of the AP2-adaptor complex (Chapman et al., 1998, Haucke et al., 2000) and mediates internalization of a chimeric construct from the plasma membrane of heterologeously transfected cells (Grass et al., 2004). At the postsynaptic site the subunits $\beta 3$ and $\gamma 2$ of the ionotrophic $\mathrm{GABA}_{\mathrm{A}^{-}}$receptor contain a basic AP-2 binding motif within an intracellular loop located between their third and forth transmembrane domain (J. Kittler, submitted).

A basic sorting motif is also present in the cytoplasmic tail of the excitatory AMPA receptor subunit GluR2. The dibasic cluster is located within the membrane proximal portion of the cytoplasmic tail (Lee et al., 2002). Competition experiments and mutational analysis have revealed that the binding sites for NSF and AP-2 in the GluR2 cytoplasmic tail are overlapping but not identical (Nishimune et al., 1998; Lee et al., 2002). Binding of the AP-2 complex to the GluR2 cytoplasmic tail has a physiological implication in AMPA receptor endocytosis upon NMDA-induced LTD in cultured hippocampal neurons (Lee et al., 2002).

Work on recognition of synaptotagmin 1 by $\mu 2$-adaptin (Haucke et al., 2000) has shown that the synaptotagmin 1 binding site in $\mu 2$ is distinct from the recognition site of tyrosine based sorting motifs which locates to a hydrophobic pocket in subdomain A. A recent study from Diviani et al. (2003) describes binding of the $\alpha_{1}$-adrenergic receptor to the $\mathrm{N}$-terminal portion and subdomain $\mathrm{B}$ of $\mu 2$-adaptin via a basic cluster comprised of eight arginines. Taken together these findings suggest that the $\mu 2$ subdomain $B$ harbours a putative recognition site for basic endocytic sorting motifs on its surface. Binding may thus occur via electrostatic interactions between the basic sorting motif and $\mu 2$. Up to now no detailed structural information on the recognition mechanism is available.

\section{Aims of the project}

Internalization of proteins from the synaptic plasma membrane by means of clathrin-mediated endocytosis is involved in the regulation of signal transmission at both sides of the synapse. Recent work by Lee et al. (2002) has shown that recognition of the AMPA receptor subunit GluR2 by the heterotetrameric clathrin-adaptor AP-2 is critically dependent on a dibasic cluster within the GluR2 cytoplasmic tail. This interaction plays a role in the NMDA-induced internalization of AMPARs from the postsynaptic active zone in hippocampal longterm depression LTD.

Based on the hypothesis that similar endocytic mechanisms may operate at both sides of the synapse we have aimed at the biochemical characterization of the interaction between the AMPA-type glutamate receptor and the clathrinadaptor complex AP-2.

To this aim we have employed biochemical and cell biological strategies in-vitro and in living cells.

In order to shed light on the biogenesis pathway of SVs during neuronal differentiation we have developed and initially characterized a differentiation system based on embryonic stem cells. 


\section{Material and Methods}

\subsection{Materials}

\subsubsection{Plasmids, DNA constructs and oligonucleotides}

All oligonucleotides were synthesized at MWG Ebersberg.

The vectors pcHA2 and pcFLAG were constructed by Yasuo Nemoto and contain the multiple cloning site from pbluescript (stratagene La Jolla USA) and a pGEX4-T1 backbone. The plasmid pCDNA 3.1 was purchased from invitrogen (Carlsbad, USA). The bacterial expression vector pet28a was obtained from Novagen () and pGEX4T1 from Amersham Pharmacia (Uppsala Sweden).

Plasmid constructs containing the AMPA receptor subunit GluR1, GluR2 and GluR3 cytoplasmic tails and point mutants of the GluR2 cytoplasmic tail K844A, R845A, K847A, V848A, K844 and the peptide K844 to Q853 in pGEX4T1 vector were kindly provided by the group of Morgan Sheng, New York (Lee et al. 2002). A full length construct of the wildtype GluR2 carrying an internal HA tag in the vector pGW1 (British Biotech) was also a kind gift from the group of Morgan Sheng (Man et al. 2000).

The HA GluR2 lacking the cytoplasmic tail was cut out of the full length contruct by Hind III, EcoRI digest and ligated into pcFLAG vector containing either the pep2r or pep2M motif to obtain either HA-GluR2pep2r or pep2M.

HA-GluR2 $\Delta$ cytoplasmic tail in pcFLAG was generated by amplification of a truncated rat HA-GluR2 containing a stop codon before its cytoplasmic tail at base 2502 of the full length DNA sequence by PCR and cloning it Hind III / EcoRI into pcFLAG.

FLAG-tagged fusion constructs in which the synaptotagmin $1 \mathrm{C} 2 \mathrm{~B}$ domain was replaced by the AMPA receptor basic sorting motif or a mutant motif were generated as described: Duplexes of annealed and phosphorylated DNA oligonucleotides encoding the pep2r or pep2M motifs were EcoRI / Xbal cloned into pcFLAG.

The sequences of the annealed oligonucleotides were as follows:

\begin{tabular}{|l|l|}
\hline oligonucleotide & sequence \\
\hline pep2r-EcoRI-for & aat tca aac gaa tga agg tgg caa aga atc cac agt gat \\
\hline pep2r-Xba-rev & cta gat cac tgt gga ttc ttt gcc acc ttc att cgt ttg \\
\hline pep2M-EcoRI-for & aat tcg cag caa tga agg tgg caa aga atc cac agt gat \\
\hline pep2M-Xbal-rev & cta gat cac tgt gga ttc ttt gcc acc ttc att gct gcg \\
\hline
\end{tabular}

Table: $3 A$

Synaptotagmin $1 \triangle \mathrm{C} 2 \mathrm{~B}$ chimeras were generated by $\mathrm{PCR}$ cloning of a synaptotagmin $1 \triangle \mathrm{C} 2 \mathrm{~B}$ fragment into Bam $\mathrm{HI}$ and EcoRI digested either pcFLAG vector carrying the pep2 $r$ or pep2M insert. To obtain FLAG-tagged synaptotagmin $1 \triangle \mathrm{C} 2 \mathrm{~B}$ a stop codon was inserted by PCR at base 798 of the full length rat synaptotagmin 1 DNA sequence and the fragment was EcoR1 / Bam $\mathrm{H} 1$ cloned into pcFLAG.

The $\mu 2$-adaptin $\Delta 157$ construct in pet28a and the full length construct carrying an internal HA-tag in pcDNA3 were made by G. Rohde (Rohde et al.; JCB).

$\mathrm{N}$-terminal HA-tagged $\mu 2$-adaptin was obtained by PCR cloning of a $\mu 2-$ adaptin $\Delta 157$ into EcoRI and Xho I digested pcHA vector. 
To block clathrin-mediated endocytosis by a dominant negative approach constructs of the amphiphysin BC domain either wildtype or mutant in pcHA generated by N. Jung were used.

For characterization of a putative binding site for basic sorting motifs in $\mu 2-$ adaptin point mutations were inserted into the full length construct and the $\mu 2-$ adaptin $\triangle 157$ construct in pGEX4T1 using the QuikChange site-directed mutagenesis kit purchased from stratagene, LaJolla USA. The mutant $\mu 2$ b1 contained the following exchanges of acidic surface residues E320N, E348N and E391K. In the second construct $\mu 2$ b2 the mutations E295N, E369L and E371N were inserted.

Sequences of the mutagenic primers were as follows:

\begin{tabular}{|c|c|}
\hline oligonucleotide & sequence \\
\hline $\mu 2$ b1 E320N & ctg gcc cag aag att caa gtg agg atc ccg act c \\
\hline$\mu 2 \mathrm{~b} 1 \mathrm{E} 348 \mathrm{~N}$ & gta caa ggc cag cag caa cgc cat tgt atg gaa g \\
\hline H2 b1 E391K & cca ttt cca tga act tta aag tgc cat tcg cgc c \\
\hline$\mu 2$ b2 E295 N & cat tgg tgc gga acg tgg ggc gca cc \\
\hline $\begin{array}{lll}\mu 2 & \text { b2 } & \text { E369L } \\
\text { E371N }\end{array}$ & gat cag cgc act gat tca gct cct gcc cac $\mathrm{c}$ \\
\hline
\end{tabular}

Table: 3B

Short interfering siRNAs to knock-down $\mu 2$-adaptin (Motley et al., JBC) in HEK 293 cells and a luciferase control siRNA were synthesized at MWG Biotech, Ebersberg.

The sequences of the sense-strands were as follows:

\begin{tabular}{|l|l|}
\hline siRNA & sense strand sequence \\
\hline human $\mu 2$-adaptin & gug gau gcc uuu cgg guc att \\
\hline 2 c 18 & uga cga cag cac cuc cuu att \\
\hline
\end{tabular}

Table: 3C

Expression of synaptic vesicle protein mRNAs during neuronal differentiation was assayed by RT-PCR using the following primers:

\begin{tabular}{|c|c|}
\hline protein & sequence \\
\hline$\beta$-actin forward & atc tgg cac cac acc ttc tac aat gag ctg cg \\
\hline reverse & cgt cat act cct gct tgc tga tcc aca tct gc \\
\hline synaptobrevin / VAMP 2 forward & gca cct cct cca aat cct acc agt \\
\hline reverse & aaa cga tga tga tga tga gga tga \\
\hline TI-VAMP / VAMP 7 forward & cca ttc ttt ttg ccg ttg ttg c \\
\hline reverse & gtt cgt cca ctt ggg ctt gag \\
\hline synapsin 1 forward & gca cac cga ctg ggc aaa ata ctt \\
\hline reverse & cct tga cct tgc cca tcc cag aat \\
\hline synaptophysin forward & ttt gcc atc ttc gcc ttt gct acg \\
\hline reverse & tgg cca tct tca cat cgg aca gg \\
\hline synaptotagmin 1 forward & tct ccc tcc get acg tcc cta ctg \\
\hline reverse & gca tgg cat caa cct cct cct cta \\
\hline SV 2 forward & gat ctc gag tat tcc cga agg tcc tac \\
\hline reverse & aag tct aga tta gat gcg ccg gta ctc tgg \\
\hline dynamin 1 forward & gga gcc cgc atc aat cgt atc ttt \\
\hline reverse & ggt atg gcc ctc ttc ttg ttc ag \\
\hline AP-2 $\mu 2$-adaptin forward & cgg ggt acc atg atc atc ctg cct ttc cgg \\
\hline reverse & cgc tct aga tca tgg cac ctc aaa gtt cat \\
\hline AP-3 $\sigma$-adaptin forward & gca att gga tcc atg gat aag gtg cac tac \\
\hline reverse & gtt ctaq gaa ttc atg atg atg atg atg atg tca gac \\
\hline
\end{tabular}




\begin{tabular}{|r|r|}
\hline stonin 2 forward & gct ctc aag acc att ccc ac \\
\hline reverse & gag tca gcc cca gaa gcc tgg cca c \\
\hline arf 6 forward & cta ggc atg cat ggg gaa ggt gct atc c \\
\hline reverse & gct gcc gcg cgg cac cag aga tt gta gtt aga cgt \\
\hline HMG-CoA-reductase forward & gca gat tct ggc agt cag tgg g \\
\hline reverse & cca tca agg aca gct cac cag \\
\hline PM-ganglioside sialidase forward & tca att ggc aaa ggt gct act cag \\
\hline reverse & ggg ctg gta cag tct tca cag ctc agg acc \\
\hline
\end{tabular}

Table 3E

\subsubsection{Synthetic peptides used in for pulldown experiments}

Synthetic peptides comprising various endocytic sorting motifs or mutated motifs were applied in a concentration of 1 up to $100 \mu \mathrm{M}$ in pulldown experiments with GST-GluR cytoplasmic tails immobilized on glutathione sepharose and TritonX-100 extracted ratbrain homogenate or purified soluble $\mu 2$-adaptin. The synthetic peptides comprised the following sequences:

\begin{tabular}{|l|l|}
\hline peptide & sequence \\
\hline pep2r & KRMKVAKNPQ \\
\hline pep KR & KRLKKKKTTIKK \\
\hline pep KKAA & KRLKKTTIKK \\
\hline
\end{tabular}

Table 3F

\subsubsection{Antibodies and endocytosis ligands}

Monoclonal antibodies against synaptic vesicle proteins $\alpha$-synaptobrevin 2 clone 69.1; $\alpha$-synaptotagmin 1 clones 41.1 and 64.04 and $\alpha$-synaptophysin were purchased from synaptic systems, Göttingen. The $\alpha$-synaptobrevin 2 clone 69.1 and $\alpha$ - synaptotagmin 1 clone 41.1 were diluted $1: 250$ and $\alpha$ synaptophysin $1: 100$ for immunocytochemistry.

The antibody against SV 2 clone $\mathrm{C} 10 \mathrm{H} 44$ a highly glycosylated synaptic vesicle protein was a kind gift from the de Camilli lab and was diluted $1: 250$ for immunofluorescence staining. For detection of AP-2 subunits on immunoblots the $\alpha-\alpha_{C}$-adaptin antibody clone AC1M11 purchased from Affinity Bioreagents (Golden USA) was diluted 1 : 100. Monoclonal AP-6 antibody directed agains $\alpha-$ adaptin was a kind gift of $\mathrm{M}$. S. Robinson, Cambridge and used in a 1:250 dilution for immunocytochemistry. $\alpha-\beta 1 / 2$-antibody purchased from Transduction Laboratories was applied in a 1:5000 dilution to detect $\beta$-adaptin on immunoblots.

Heat shock protein 70 was detected on immunoblots using the monoclonal antibody from Affinity Bioreagents, Golden USA in a dilution of $1: 1000$. The monoclonal AP $6 \alpha-\alpha$-adaptin antibody was used $1: 50$ for labelling of clathrincoated pits in fixed tissue. To detect clathrin-heavy chain on immunoblots the monoclonal antibody TD 1 a generous gift from the de Camilli lab was diluted 1:5. Monoclonal antibodies $\alpha$-Eps15; $\alpha-\mu 2$-adaptin AP50 and $\alpha$-PIP5-kinase $1 \gamma$ were purchased from BD Transduction Labs (Palo Alto USA). For identification of corresponding proteins on immunoblots they were used in a dilution of $1: 250$. 
Huntingtin interacting protein HIP1 $\mathrm{R}$ was detected on immunoblots using a monoclonal antibody $\alpha$-HIP1R (BD Transduction Labs) diluted $1: 100 . \alpha-\gamma-$ adaptin to identify the AP-1 subunit was purchased from BD Biosciences and applied 1:1000 on inmmunoblots. For immunofluorescence labelling of the Golgi apperatus monoclonal $\alpha-G M 130$ (BD Biosciences) was used in a 1:100 dilution.

HA-tagged proteins were detected by monoclonal $\alpha$-HA F7 (Santa Cruz, Santa Cruz USA) diluted $1: 100$ for immunocytochemistry and $1: 250$ for western blotting.

Polyclonal $\alpha-\mathrm{HA}$ antibody $\mathrm{Y}-11$ (Santa Cruz) was applied in a 1:100 dilution for immunofluorescence.

FLAG-tagged proteins were labelled with monoclonal $\alpha$-FLAG antibody M2 purchased from Sigma, Henningsdorf diluted 1 : 200 for immunocytochemistry.

For antibody internalization assays in transfected Cos 7 cells $\alpha$-HA antibody F7 was applied on the cells in a 1:50 dilution $\alpha$-FLAG antibody M2 was diluted 1 : 500.

Polyclonal antibodies against synaptobrevin 2 and synaptotagmin 1,2 were purchased from synaptic systems, Göttingen and applied 1 : 100 for immunocytochemistry. The GTPase dynamin 1 was detected on immunoblots by polyclonal $\alpha$-dynamin 1 DG 1 antibody diluted 1 : 1000 kindly provided by the de Camilli Lab. Early endosomes were labelled for immunocytochemistry using a polyclonal $\alpha$-EEA1 antibody O7JF3 1 : 50 a generous gift from $M$ Zervial. Polyclonal $\alpha$-Rab 11 purchased from Zymed, Berlin was used $1: 50$ to stain recycling endosomes for immunocytochemistry.

Unspecific staining by secondary Alexa-dye conjugated goat anti-mouse for immunocytochemistry was prevented by blocking of the sample with unlabeled Goat anti-Mouse IgG (dianova, Hamburg) in a 1:10 dilution.

Monoclonal primary antibodies in immunocytochemistry were detected with Alexa488 or Alexa594 conjugated goat anti-Mouse secondary antibody applied in a $1: 150$ dilution purchased from Molecular Probes. For labeling of polyclonal primary antibodies Alexa 488 or Alexa 594 conjugated secondary goat antiRabbit antibody was applied in a 1: 150 dilution on fixed tissues. Alexa350 or Cy5 conjugated goat anti-rabbit were diluted 1:50 for detection of primary polyclonal antibodies.

Endocytosis assays were performed using Alexa488-conjugated transferrin purchased from Molecular Probes.

Primary monoclonal antibodies on western blots were labeled with horse radish peroxidase conjugated secondary goat anti-mouse antibody (dianova) in a 1 : 5000

dilution. Horse radish peroxidase conjugated secondary goat anti-rabbit antibody was applied 1: 10000 to label primary polyclonal antibodies on immunoblots. 


\subsection{Molecular biological methods}

\subsubsection{RNA isolation and RT-PCR}

Cultured cells from a $5 \mathrm{~cm}$ dish were harvested by trypsinization and the cell pellet was collected by centrifugation. The cell pellet was washed twice with PBS. For RNA isolation from the cell pellet a Purescript RNA isolation kit from Gentra Systems (Minneapolis USA) was used according to the manufacturer's instructions.

The DNA concentration was determined photometrically at $260 \mathrm{~nm}$ in an Eppendorf Biophotometer.

$1 \mu \mathrm{g}$ of RNA and $2 \mathrm{U}$ of DNAse 1(Sigma, Henningsdorf) were used for DNAse digest in $1 \times$ DNAse digestion buffer $\left(50 \mu \mathrm{M} \mathrm{NaAC} ; 5 \mathrm{mM} \mathrm{MgSO}_{4}\right)$. The reaction was incubated for $15 \mathrm{~min}$ at room temperature (RT) and the enzyme was inactivated for $15 \mathrm{~min}$ at $65^{\circ} \mathrm{C}$.

Quality of isolated RNA was checked by running samples on a $1 \%$ agarose gel at $100 \mathrm{mV}$ for $5 \mathrm{~min}$. After staining with ethidium bromide a double of ribosomal DNA was visible on the gel whereas a band of genomic DNA migrating above the RNA bands should not appear after DNAse 1 treatment.

For reverse transcription (RT) of RNA reverse transcriptase Superscript II purchased from Gibco BRL was used. The reaction contained 500 ng of RNA; $20 \mu \mathrm{g} / \mathrm{ml}$ hexameric random primer (Gibco BRL), $0.4 \mu \mathrm{M}$ dNTPs (Gibco BRL) and water up to a volume of $13 \mu \mathrm{l}$. The mixture was heated to $65^{\circ} \mathrm{C}$ to denature the RNA and quickly chilled on ice.

First Strand Buffer (Gibco BRL) and 4 mM DTT were added and samples were incubated for $2 \mathrm{~min}$ at $42{ }^{\circ} \mathrm{C}$. After that $200 \mathrm{U}$ of Superscript II reverse transcriptase are added and the reaction was incubated for $60 \mathrm{~min}$ at $42^{\circ} \mathrm{C}$. The enzyme was heat inactivated afterwards for $15 \mathrm{~min}$ at $70^{\circ} \mathrm{C} .1 \mu \mathrm{l}$ of the cDNA was used as template for PCR reactions.

Oligonucleotides specific for the DNA sequence of certain synaptic vesicle proteins and $\beta$-actin were designed with the primer select program from DNASTAR.

Taq polymerase for RT-PCR and the according buffer were purchased from Genaxxon, Berlin. The reaction contained $1 \mu \mathrm{l}$ of template CDNA, $1 \times$ Taq reaction buffer, $200 \mu \mathrm{M}$ dNTPs, $500 \mu \mathrm{M}$ of forward and reverse primer and $1 \mathrm{U}$ Taq Polymerase. Polymerase chain reaction (PCR) was carried out according to the following protocol: Denaturation of the template DNA at $95^{\circ} \mathrm{C}$ for $2 \mathrm{~min}$; 30 cycles of denaturation for $30 \mathrm{sec}$ at $95{ }^{\circ} \mathrm{C}$, primer annealing for $30 \mathrm{sec}$ at 55 ${ }^{\circ} \mathrm{C}$ and polym erization for $1 \mathrm{~min}$ at $72{ }^{\circ} \mathrm{C}$. A final round of extension was run at $72{ }^{\circ} \mathrm{C}$ for $5 \mathrm{~min}$

PCR products of the synaptic vesicle protein encoding CDNA or $\beta$-actin CDNA were detected by UV-illumination of ethidium bromide stained agarose gels.

\subsubsection{Annealing and phosphorylation of single stranded oligonucleotides}

Oligonucleotide annealing was used for cloning of short DNA fragments up to 50 base pairs. The primers were designed with restriction enzyme site overhangs for ligation into linearized vector. 
For the annealing reaction $10 \mathrm{mM}$ forward and reverse primer in $100 \mathrm{mM} \mathrm{NaCl}$, $10 \mathrm{mM}$ Tris $\mathrm{pH} 7.4$ were denatured for $5 \mathrm{~min}$ at $95^{\circ} \mathrm{C}$ and then slowly cooled down to room temperature in $2{ }^{\circ} \mathrm{C}$ steps.

$1 \mathrm{mM}$ duplex DNA was then $5^{\prime}$-phosphorylated in $1 \times$ T4 polynucleotide kinase buffer, $1 \mathrm{mM}$ ATP and $10 \mathrm{U}$ of polynucleotide kinase (New England Biolabs, Beverly USA) for $30 \mathrm{~min}$ at $37^{\circ} \mathrm{C}$. The reaction was $10 \mathrm{x}$ diluted with water. For ligation reaction $0.5 \mu$ of phosphorylated duplex DNA were used.

\subsubsection{PCR amplification of DNA fragments}

Oligonucleotides containing a restriction enzyme digestion site were designed specific for the DNA target sequence of the template. PCR was carried out using Vent Polymerase from New England Biolabs (Beverly USA) when proof reading activity was required.

The reaction conditions were as follows: $1 \times$ Thermo Pol Reaction buffer from NEB $200 \mu \mathrm{M}$ dNTPs; $500 \mu \mathrm{M}$ of forward and reverse primer; $1 \mathrm{U}$ Vent Polymerase and $10 \mathrm{ng}$ of template DNA.

PCR was performed according to the following protocol: Denaturation of the template DNA at $95{ }^{\circ} \mathrm{C}$ for $2 \mathrm{~min} ; 25$ cycles of denaturation for $30 \mathrm{sec}$ at $95{ }^{\circ} \mathrm{C}$, primer annealing for $30 \mathrm{sec}$ at $55^{\circ} \mathrm{C}$ and polymerization for $1 \mathrm{~min}$ at $72{ }^{\circ} \mathrm{C}$. A final round of extension was run at $72{ }^{\circ} \mathrm{C}$ for $5 \mathrm{~min}$.

PCR fragments were purified by agarose gel electrophoresis and gel extraction of DNA bands or using the E.Z.N.A. Cycle-Pure-Kit purchased from peqlab, Erlangen.

\subsubsection{Colony PCR}

To select positive clones by colony PCR colonies were picked from an LBampicillin or kanamycin agar plate to a 96-well-plate containing LB-medium and $100 \mu \mathrm{g} / \mathrm{ml}$ ampicillin or $50 \mu \mathrm{g} / \mathrm{ml}$ kanamycin. The plate was incubated for $2 \mathrm{~h}$ at $37^{\circ} \mathrm{C}$. $1 \mu \mathrm{l}$ of the bacteria suspension was used as template for PCR. The reaction contained 1 x Taq buffer from Genaxxon, Berlin; $200 \mu \mathrm{M}$ dNTPs; $1 \mathrm{U}$ Taq Polymerase from Genaxxon and $500 \mu \mathrm{M}$ of a forward primer according to a sequence in the vector backbone and a reverse primer specific for the insert sequence.

PCR was carried out according to the following protocol: Denaturation of the template DNA at $95^{\circ} \mathrm{C}$ for $5 \mathrm{~min}$; 30 cycles of denaturation for $30 \mathrm{sec}$ at $95^{\circ} \mathrm{C}$, primer annealing for $30 \mathrm{sec}$ at $55^{\circ} \mathrm{C}$ and polymerization for $1 \mathrm{~min}$ at $72{ }^{\circ} \mathrm{C}$. A final round of extension was run at $72{ }^{\circ} \mathrm{C}$ for $5 \mathrm{~min}$.

\subsubsection{PCR mutagenesis of plasmid DNA}

For insertion of mutations oligonucleotides including the desired mutation site were designed. Site directed mutagenesis was carried out using the QuikChange site-directed mutagenesis kit purchased from stratagene, LaJolla USA according to the manufactorer's instructions. The PCR mutagenesis reaction contained $5 \mu \mathrm{l} 1 \times$ reaction buffer (Stratagene, LaJolla USA); 50 ng of template DNA; $125 \mathrm{ng}$ of forward and reverse primers; dNTPs and $2.5 \mathrm{Pfu}$ Turbo Polymerase (Stratagene, LaJolla USA). The PCR reaction started with a $30 \mathrm{sec}$ denaturing step at $95^{\circ} \mathrm{C}$ for $30 \mathrm{sec}$ followed by 16 cycles of a $30 \mathrm{sec}$ 
denaturing step at $95{ }^{\circ} \mathrm{C}$ for $30 \mathrm{sec}$; a primer annealing step at $55{ }^{\circ} \mathrm{C}$ for 1 minute and a polymerization step at $68{ }^{\circ} \mathrm{C}$ for 1 minute per $1 \mathrm{~kb}$ of plasmid length. The reaction tube was chilled on ice and Dpn I ( $1 \mathrm{U})$ digested for $1 \mathrm{~h}$ at $37^{\circ} \mathrm{C}$. $10 \mu \mathrm{l}$ of the reaction were transformed into chemically competent E. coli cells.

\subsubsection{Agarose gel electrophoresis of DNA fragments}

PCR fragments and vector restriction digests were purified by agarose gel electrophoresis. Depending on DNA fragment size $1 \%$ or $2 \%$ agarose gels (Karl Roth, Karlsruhe) in 1 x TBE buffer were run for about $30 \mathrm{~min}$ at $100 \mathrm{mV}$. Samples were diluted in 1 x DNA loading buffer (Fermentas, St. Leon-Rot) and applied on the gel together with $0.5 \mu \mathrm{g}$ of $50 \mathrm{bp}$ or $1 \mathrm{~kb}$ DNA ladder standard (Fermentas, St. Leon-Rot) to estimate the DNA fragment size.

The agarose gel was stained in ethidium bromide containing TBE buffer and DNA bands were visualized under UV-light. Bands of interest were cut out and the DNA was purified from the agarose by a QUIAEX II gel purification kit purchased from QUIAGEN, Hilden.

\subsubsection{Restriction digest of PCR fragments and vector}

Restriction enzymes, the according buffers and BSA were purchased from New England Biolabs (Beverly USA). The purified DNA fragment was digested in a total volume of $60 \mu \mathrm{l}$ with the recommended $1 \times$ restriction buffer, $100 \mu \mathrm{g} / \mathrm{ml}$ $\mathrm{BSA}$ and $40 \mathrm{U}$ of the restriction enzyme. The reaction was incubated for $4 \mathrm{~h}$ at $37{ }^{\circ} \mathrm{C}$. Double digests were performed in the recommended buffer conditions. The enzymes can be inactivated at $75^{\circ} \mathrm{C}$ for $15 \mathrm{~min}$.

For vector digest $6 \mu \mathrm{g}$ of DNA plasmid were incubated with the recommended 1 $x$ restriction buffer, $100 \mu \mathrm{g} / \mathrm{ml}$ BSA and $6 U$ of the restriction enzyme in a total reaction volume of $60 \mu \mathrm{l}$. For isolation of linearized vector the restriction enzyme digest was run on an agarose gel.

For screening of clones DNA was isolated from $2 \mathrm{ml}$ overnight culture. $5 \mu \mathrm{l}$ of the isolated DNA were digested with the recommended $1 \times$ restriction buffer, $100 \mu \mathrm{g} / \mathrm{ml}$ BSA and $5 \mathrm{U}$ of the restriction enzyme in a total volume of $10 \mu \mathrm{l}$ for 2 h at $37^{\circ} \mathrm{C}$.

\subsubsection{Dephosphorylation and ligation}

For dephosphorylation of linearized plasmid DNA $0.5 \mathrm{U}$ of calf intestine phosphatase CIP (Roche Mannheim) were added to the ice chilled restriction digest reaction immediately after heat inactivation of the restriction enzyme. The sample was incubated for $10 \mathrm{~min}$ at $37^{\circ} \mathrm{C}$ and seperated on an agarose gel immediately afterwards. T4 DNA ligase and according buffers are purchased from Roche Mannheim. Amounts of insert and vector DNA were estimated by a known DNA ladder standard (Fermentas) from an ethidium bromide stained agarose gel. For the ligation reaction a threefold excess of insert DNA was used. The insert DNA was incubated with linearized, dephosphorylized vector 
DNA, $1 \times$ T4 ligase reaction buffer and $1 \cup$ T4 ligase in a total volume of $20 \mu \mathrm{l}$ for $3 \mathrm{~h}$ at $16{ }^{\circ} \mathrm{C}$ or at $4{ }^{\circ} \mathrm{C}$ overnight.

$10 \mu \mathrm{l}$ of the ligation reaction were transformed into chemically competent E. coli cells.

\subsubsection{Culture of E. coli strains and transformation into chemically competent E. coli}

The E. coli strain TOP10 was purchased from Invitrogen (Carlsbad USA). For protein expression plasmids were transformed into BL21-Codon Plus competent cells from Stratagene (LaJolla, USA). The cells were inoculated into $50 \mathrm{ml}$ LBmedium (1 \% Tryptone (Karl Roth, Karlsruhe); $0.5 \%$ yeast extract (Karl Roth, Karlsruhe); $1 \% \mathrm{NaCl}$ ) and cultured at $37{ }^{\circ} \mathrm{C}$ and $300 \mathrm{rpm}$ in a shaker until an $\mathrm{OD}_{600}$ of 0.4 was reached. Cells were harvested by centrifugation for $10 \mathrm{~min}$ at $4{ }^{\circ} \mathrm{C}$ and $4000 \mathrm{rpm}$. The cell pellet was resuspended in $10 \mathrm{ml}$ sterile ice-cold 0.1 $\mathrm{M} \mathrm{CaCl}_{2}$. The cell suspension was incubated for 15 min up to $4 \mathrm{~h}$ on ice. Cells were collected by centrifugation afterwards. The cell pellet was resuspended in $2 \mathrm{ml} 0.1 \mathrm{M} \mathrm{CaCl}_{2}$. Glycerole was added to a final concentration of $10 \%$. Aliquots of the cell suspension were frozen in liquid nitrogen and were stored at $-80^{\circ} \mathrm{C}$.

$10 \mu \mathrm{l}$ of a ligation reaction were incubated with $100 \mu \mathrm{l}$ of chemically competent E. coli cells for 30 min on ice. A heat shock was performed for $90 \mathrm{sec}$ at $42{ }^{\circ} \mathrm{C}$ and the cells were chilled on ice for $2 \mathrm{~min}$. The complete transformation was plated on a LB-agar-plate containing either $100 \mu \mathrm{g} / \mathrm{ml}$ Ampicillin or $50 \mu \mathrm{g} / \mathrm{ml}$ kanamycin and incubated overnight at $37^{\circ} \mathrm{C}$.

\subsubsection{Isolation and purification of plasmid DNA}

For restriction digest screens crude DNA was isolated from $2 \mathrm{ml}$ of an overnight culture. The cells were harvested by centrifugation and resuspended in $5 \times \mathrm{TE}$ buffer (50 mM Tris-HCl; 5 mM EDTA pH 8.0) containing RNase. $200 \mu$ of lysis buffer ( $1 \%$ SDS; $0.2 \mathrm{M} \mathrm{NaOH}$ ) were added and the reaction mixed by inverting the tube. For neutralization $150 \mu \mathrm{l} 3 \mathrm{M} \mathrm{KAc} \mathrm{pH} 4.8$ were added. Proteins and genomic DNA were pelleted by centrifugation at maximum speed for $10 \mathrm{~min}$. The supernatant was transferred into a reaction tube containing $800 \mu \mathrm{l}$ of isopropanole and the DNA pellet was isolated by centrifugation at maximum speed for $10 \mathrm{~min}$. The DNA pellet was washed with $500 \mu \mathrm{l}$ of $70 \%$ ethanole, airdried and resuspended in $50 \mu \mathrm{l}$ water.

For sequencing DNA from $5 \mathrm{ml}$ of an overnight culture was purified using the Wizard Plus SV Miniprep DNA Purification System purchased from Promega, Mannheim according to the manufactorers instructions.

DNA from $50 \mathrm{ml}$ to $150 \mathrm{ml}$ overnight culture was isolated using the QUIAGEN Midiprep Kit purchased from QIAGEN, Hilden. 


\subsubsection{DNA sequencing}

For DNA sequencing $1 \mu \mathrm{g}$ of Wizard Miniprep purified DNA was airdried. The sequencing reaction was carried out at MWG, Ebersbach. Sequencing results were aligned with $\mathrm{NCBI}$ database entries using the MegAlign programm from DNASTAR. The sequence results were checked for mutations, correctness of the reading frame, presence of start and stop codons and restriction enzyme sites and for correct insertion of point mutations or tags.

\subsection{Protein biochemical methods}

\subsubsection{Expression and purification of GST-tagged proteins in the E. coli strain BL21}

For protein expression in E. coli $50 \mathrm{ml}$ of an overnight culture of BL21 cells were inoculated into 1 I 2xYT medium (2 \% Trypton (Karl Roth, Karlsruhe); 1 \% yeast extract (Karl Roth, Karlsruhe); $1 \% \mathrm{NaCl} ; 100 \mu \mathrm{g} / \mathrm{ml}$ Ampicillin). The cells were cultured at $30{ }^{\circ} \mathrm{C}$ shaking at $200 \mathrm{rpm}$ until an $\mathrm{OD}_{600}$ of 0.8 was reached.

Protein expression was induced by adding $0.5 \mathrm{mM}$ isopropylthiogalactosid( Karl Roth, Karlsruhe). The cells were incubated for another $3 \mathrm{~h}$ at $30{ }^{\circ} \mathrm{C}$ and 200 rpm. The cell pellet was collected by centrifugation for $15 \mathrm{~min}$ at $5000 \mathrm{rpm}$ and $4{ }^{\circ} \mathrm{C}$ in a Beckman JA-14 rotor and resuspended in $40 \mathrm{ml}$ ice-cold PBS $1 \mathrm{mM}$ PMSF.

The cells were incubated with lysozyme (Karl Roth, Karlsruhe) and $250 \mathrm{U}$ Benzonase (Sigma, Henningsdorf) for $10 \mathrm{~min}$ on ice. The cell suspension was sonicated twice for $30 \mathrm{sec}$ at $50 \%$ duty and $60 \%$ power and solubilized with 1 $\%$ Triton X-100 (Sigma, Henningsdorf) for 10 min on ice. Cell debris was isolated by centrifugation for $15 \mathrm{~min}$ at $15000 \mathrm{rpm}$ and $4{ }^{\circ} \mathrm{C}$ in Beckmann JA-20 rotor. The supernatant was transferred into a $50 \mathrm{ml}$ Falcon tube and $1 \mathrm{ml}$ of PBS washed GST-bind resin (Novagen, Madison USA) was added. The beads were incubated for $2 \mathrm{~h}$ at $4{ }^{\circ} \mathrm{C}$ on a wheel and GST-bind resin was isolated by centrifugation at $3000 \mathrm{rpm}$ and $4{ }^{\circ} \mathrm{C}$. The resin was washed three times with 10 $\mathrm{ml}$ PBS and resuspended in $500 \mu \mathrm{l}$ PBS $1 \mathrm{mM}$ PMSF. Total protein concentration was determined by a Bradford assay.

The GST-fusion protein can be eluted by incubation with $20 \mathrm{mM}$ reduced glutathione; $20 \mathrm{mM}$ Tris $\mathrm{HCl} \mathrm{pH} \mathrm{8.0;} 100 \mathrm{mM} \mathrm{NaCl}$ for $1 \mathrm{~h}$ at room temperature on wheel.

\subsubsection{Expression and purification of His $_{6}$-tagged proteins in E. coli BL21}

For protein expression in E. coli $50 \mathrm{ml}$ of an overnight culture of BL21 cells were inoculated into 1 I 2xYT medium (2 \% Trypton (Karl Roth, Karlsruhe); 1 \% yeast extract (Karl Roth, Karlsruhe); $1 \% \mathrm{NaCl} ; 100 \mu \mathrm{g} / \mathrm{ml}$ Ampicillin). The cells were cultured at $30{ }^{\circ} \mathrm{C}$ shaking at $200 \mathrm{rpm}$ until an $\mathrm{OD}_{600}$ of 0.8 was reached. 
Protein expression was induced by adding $0.5 \mathrm{mM}$ isopropylthiogalactosid( Karl Roth, Karlsruhe). The cells were incubated for another $3 \mathrm{~h}$ at $30{ }^{\circ} \mathrm{C}$ and 200 rpm. The cell pellet was collected by centrifugation for $15 \mathrm{~min}$ at $5000 \mathrm{rpm}$ and $4{ }^{\circ} \mathrm{C}$ in a Beckman $\mathrm{JA}-14$ rotor and resuspended in $40 \mathrm{ml}$ ice-cold PBS; $1 \mathrm{M}$ $\mathrm{NaCl} ; 1 \mathrm{mM}$ PMSF.

The cells were incubated with lysozyme (Karl Roth, Karlsruhe) and $250 \mathrm{U}$ Benzonase (Sigma, Henningsdorf) for $10 \mathrm{~min}$ on ice. The cell suspension was sonicated twice for $30 \mathrm{sec}$ at $50 \%$ duty and $60 \%$ power and solubilized with 2 $\%$ CHAPS (Karl Roth, Karlsruhe) for 15 min on ice. Cell debris was isolated by centrifugation for 15 min at $15000 \mathrm{rpm}$ and $4{ }^{\circ} \mathrm{C}$ in Beckmann JA-20 rotor.

The supernatant was diluted with PBS to a final $\mathrm{NaCl}$ concentration of $300 \mathrm{mM}$ and incubated for two hours at $4{ }^{\circ} \mathrm{C}$ on the wheel with $500 \mu \mathrm{l}$ of $\mathrm{Ni}^{2+}-\mathrm{NTA}$ agarose (QIAGEN, Hilden). The $\mathrm{Ni}^{2+}$-NTA-agarose was isolated by centrifugation at $3000 \mathrm{rpm}$ and $4{ }^{\circ} \mathrm{C}$. The beads were washed three times with PBS; $300 \mathrm{mM} \mathrm{NaCl} ; 10 \mathrm{mM}$ imidazole. After washing the protein was eluted from the beads in PBS; $500 \mathrm{mM} \mathrm{NaCl} ; 300 \mathrm{mM}$ imidazole for one hour at $4{ }^{\circ} \mathrm{C}$ on the wheel.

To remove the imidazole the supernatant was dialyzed against $10 \mathrm{mM}$ HEPES $\mathrm{pH}$ 7.4: $500 \mathrm{mM} \mathrm{NaCl} ; 10 \mathrm{mM} \beta$-mercaptoethanole overnight at $4{ }^{\circ} \mathrm{C}$.

After dialysis $10 \%$ of glycerole were added to the protein solution. The concentration was determined by a Bradford assay. Aliquots of the protein solution were frozen in liquid nitrogen and stored at $-80^{\circ} \mathrm{C}$.

\subsubsection{Preperation of ratbrain extract}

Ratbrain was homogenized in 320 mM Sucrose; 4 mM HEPES; 1 mM PMSF pH 7.4 by 10 strokes at $900 \mathrm{rpm}$ using a Dounce homogenizer. The homogenate was centrifuged for $10 \mathrm{~min}$ at $3000 \mathrm{rpm}$ and $4{ }^{\circ} \mathrm{C}$ in a Beckman JA-20 rotor. The supernatant was solubilized for 15 minutes on ice with $1 \%$ Triton X-100; 120 $\mathrm{mM} \mathrm{KCl} ; 20 \mathrm{mM}$ HEPES $\mathrm{pH} 7.4 ; 2 \mathrm{mM} \mathrm{MgCl}_{2}$. The ratbrain extract was centrifuged for $30 \mathrm{~min}$ at $14000 \mathrm{rpm}$ and $4{ }^{\circ} \mathrm{C}$ in a Beckman JA-20 rotor. For clarification the supernatant was ultracentrifuged for $15 \mathrm{~min}$ at $60000 \mathrm{rpm}$ and 4 ${ }^{\circ} \mathrm{C}$ in a Beckman TLA 100.3 rotor.

Protein concentration of the supernatant was determined by Bradford assay.

\subsubsection{In-vitro-transcription-translation of proteins}

For in-vitro protein transcription translation the TNT coupled reticulocyte lysate system purchased from Promega, Madison USA was used according to the manufactorer's instructions.

The reaction contained $1 \mu \mathrm{g}$ of template DNA; $1 \times$ TNT reaction buffer; rabbit reticulocyte lysate; $20 \mu \mathrm{M}$ amino acid mixture minus methionine; RNAse inhibitor; T7 RNA polymerase and $0.6 \mu \mathrm{Ci}\left[{ }^{35} \mathrm{~S}\right]$-methionine, Amersham Biosciences Uppsala Sweden. The transcription-translation reaction was incubated for $90 \mathrm{~min}$ at $30{ }^{\circ} \mathrm{C}$. An aliquot of the reaction seperated by SDSPAGE. The comassie stained poly-acrylamide gel was dried and exposed to an autoradiography screen to check to estimate the quality and quantity of the reaction product. 


\subsubsection{Pulldown of proteins from ratbrain extract}

$1.5 \mathrm{mg}$ of TritonX-100 extracted ratbrain homogenate were incubated with 100 $\mu \mathrm{g}$ of GST-fusion protein for 90 minutes at $4{ }^{\circ} \mathrm{C}$ on a wheel.

The beads were isolated by centrifugation for $2 \mathrm{~min}$ at $1000 \mathrm{rpm}$ and $4{ }^{\circ} \mathrm{C}$. The pellet was washed three times for 5 minutes with $120 \mathrm{mM} \mathrm{KCl} ; 20 \mathrm{mM}$ HEPES $\mathrm{pH} 7.4 ; 2 \mathrm{mM} \mathrm{MgCl} ; 1 \%$ Triton X 100 and once in buffer without Triton-X 100. The beads were resuspended in $40 \mu \mathrm{l}$ of 1 x SDS-PAGE sample buffer. Affinity purification was analyzed by SDS-PAGE and immunoblotting.

\subsubsection{Pulldown of purified His- $\mu 2 \Delta 157$}

$3 \mu \mathrm{g}$ of purified His- $\mu 2 \Delta 157$ were diluted in $500 \mu \mathrm{l} 1 \times$ buffer $\mathrm{T}(150 \mathrm{mM} \mathrm{NaCl}$; 20 mM HEPES pH 7.43; 2 mM EDTA; $0.5 \%$ BSA; $1 \%$ Triton X 100; 1 mM PMSF).

The protein solution was ultracentrifuged in a Beckman TLA100.3 rotor for 15 min at $60000 \mathrm{rpm}$ and $4{ }^{\circ} \mathrm{C}$ to remove precipitates of protein.

The supernatant was incubated with $10 \mu \mathrm{g}$ of GST-fusion protein for 90 minutes at $4{ }^{\circ} \mathrm{C}$ on a wheel. Beads were isolated by centrifugation for $2 \mathrm{~min}$ at $1000 \mathrm{rpm}$ and $4{ }^{\circ} \mathrm{C}$.

The pellet was washed three times for 5 minutes in buffer $T$ without BSA and once in buffer $T$ without BSA and Triton- $X 100$. The beads were resuspended in $40 \mu \mathrm{l}$ of $1 \times$ SDS-PAGE sample buffer. Affinity purification was analyzed by SDS-PAGE and Comassie Blue staining.

\subsubsection{Pulldown of in-vitro-translated proteins}

Approximately $5 \mu \mathrm{l}$ of the in-vitro translation reaction were diluted in $500 \mu \mathrm{l} 1 \mathrm{x}$ buffer $\mathrm{T}$. The protein solution was ultracentrifuged in a Beckman TLA100.3 rotor for $15 \mathrm{~min}$ at $60000 \mathrm{rpm}$ and $4{ }^{\circ} \mathrm{C}$ to remove precipitates of protein.

The supernatant was incubated with $10 \mu \mathrm{g}$ of GST-fusion protein for 90 minutes at $4{ }^{\circ} \mathrm{C}$ on a wheel. Beads were isolated by centrifugation for $2 \mathrm{~min}$ at $1000 \mathrm{rpm}$ and $4{ }^{\circ} \mathrm{C}$.

The pellet was washed three times for 5 minutes in buffer $T$ without BSA and once in buffer $T$ without BSA and Triton-X 100. The beads were resuspended in $40 \mu$ of $1 \times$ SDS-PAGE sample buffer.

\subsubsection{SDS-PAGE and Western Blot analysis of proteins}

For SDS-PAGE protein samples are denatured in $1 \times$ sample buffer $(160 \mathrm{mM}$ Tris $\mathrm{pH}$ 6.8; $20 \% \mathrm{v} / \mathrm{v}$ glycerole; $4 \% \mathrm{w} / \mathrm{v}$ sodium dodecyl sulfate; $0.05 \% \mathrm{w} / \mathrm{v}$ bromphenole blue; $5 \% \mathrm{v} / \mathrm{v} \beta$-mercaptoethanole) for 4 min at $95{ }^{\circ} \mathrm{C}$.

The seperation gel contained $10 \%$ and the stacking gel $4.8 \%$ poly-acrylamide of a $30 \%$ stock solution purchased from Carl Roth, Karlsruhe and $4 X$ seperation buffer (1.5 M Tris $\mathrm{pH} 8.8 ; 0.4 \% \mathrm{w} / \mathrm{v}$ SDS) or $4 \times$ stacking gel buffer (0.5 M Tris $\mathrm{pH} 6.8 ; 0.4 \% \mathrm{w} / \mathrm{v}$ SDS). The polyacrylamide polymerization was 
catalyzed by $0.05 \%$ w/v ammonium persulfate (Carl Roth, Karlsruhe) and 0.015 $\%$ TEMED (Sigma, Henningsdorf).

The polyacrylamide gel electrophoresis was performed in a vertical slab gel unit in 1xSDS-PAGE buffer (25 mM Tris pH 8.3; $0.19 \mathrm{M}$ glycine; $0.1 \% \mathrm{w} / \mathrm{v}$ SDS) at $20 \mathrm{~mA}$ for 90 minutes.

The gel was blotted on a nitrocellulose membrane (Whatman Biometra, Göttingen) with 1 x blotting buffer (25 mM Tris pH 8.3; $0.19 \mathrm{M}$ glycine; $0.1 \% \mathrm{w} / \mathrm{v}$ SDS; $20 \%$ methanole) using a Biometra semi-dry blotter for $2 \mathrm{~h}$ at $1 \mathrm{~mA} / \mathrm{cm}^{2}$.

The nitrocellulose membrane was stained with $0.5 \%$ Ponceau S (Carl Roth, Karlsruhe) in $3 \% \mathrm{v} / \mathrm{v}$ acetic acid to verify successful transfer of protein.

The blotting membrane was blocked in blotto ( TBS; $5 \% \mathrm{w} / \mathrm{v}$ non fat dry milk Carl Roth, Karlsruhe) for $60 \mathrm{~min}$ at room temperature. Primary monoclonal or polyclonal antibodies were applied in TBS; $1 \%$ BSA; $0.02 \%$ sodium azide and incubated shaking for $2 \mathrm{~h}$ at room temperature. The blotting membrane was washed three times for 10 min with blotto and incubated with secondary horse radish peroxidase conjugated goat-anti-mouse 1:5000 or goat-anti-rabbit antibodies 1:10000 in blotto for $1 \mathrm{~h}$ at room temperature. The membrane was washed twice with blotto and twice with PBS.

The blot was developped with ECL detection reagent (Amersham Biosciences, Uppsala Sweden). The signal was detected by exposure of the blot for 1 min to 5 min to an X-ray film reagent (Amersham Biosciences, Uppsala Sweden).

For detection of the signal with $\left[{ }^{125} \mathrm{I}\right]$ labelled protein A (Amersham Biosciences, Uppsala Sweden) the blotting membrane was incubated with secondary nonlabelled rabbit-anti-mouse antibody 1:1000 in TBS; $1 \%$ BSA; $0.02 \%$ sodium azide for $2 \mathrm{~h}$ at room temperature if the primary antibody was monoclonal.

The blotting membrane was washed three times with blotto and incubated with $0.3 \mu \mathrm{Ci}$ of $\left[{ }^{125} \mathrm{I}\right]$ labelled protein $\mathrm{A}$ in blotto for $1 \mathrm{~h}$ at room temperature. The blotting membrane was washed three times for 15 min with blotto and once with TBS. The autoradiography signal was detected and quantified using a phosphoimager.

\subsection{Cell biological methods}

\subsubsection{Mammalian cell culture}

Cos 7 cells and human embryonic kidney (HEK 293) fibroblasts were purchased from DSMZ, Braunschweig.

PA6 (MC3T3-G2) cell line derived from mouse newborn calvaria was ordered at the RIKEN Bioressource Center, Tokio Japan.

The murine neuroblastoma cell line N1E was a kind donation from the lab of $\mathrm{H}$. Neumann at the European Neuroscience Institute, Göttingen. ES14 murine embryonic stem cells were a generous gift from N. Brose at the MPI for Experimental Medicine, Göttingen. The murine embryo carcinoma cell line P19 was purchased from the DSMZ Braunschweig.

Cell culture media, sera, antibiotics, L-glutamine stock solution and trypsinEDTA were bought from Gibco, Carlsbad USA.

HEK293 cells were grown in DMEM high Glucose containing $10 \%$ of FCS and $1 \%$ PenStrep. Cells were maintained at $37{ }^{\circ} \mathrm{C}$ and $5 \% \mathrm{CO}_{2}$ and passaged 
twice a week using trypsin-EDTA for detachment. Cos 7 fibroblasts were cultured in DMEM 1000 mg glucose / I containing 10 \% FCS and 1 \% PenStrep. Cells were maintained at $37{ }^{\circ} \mathrm{C}$ and $5 \% \mathrm{CO}_{2}$ and passaged twice a week using trypsin-EDTA for detachment.

PA6 cells were cultured in Alpha Minimum Essential Medium ( $\alpha M E M), 10 \%$ FCS and $1 \%$ PenStrep. Cells were maintained at $37{ }^{\circ} \mathrm{C}$ and $5 \% \mathrm{CO}_{2}$ and passaged once a week using trypsin-EDTA for detachment.

P19 cells were cultured in Alpha Minimum Essential Medium ( $\alpha$ MEM), $10 \%$ FCS and $1 \%$ PenStrep. Cells were maintained at $37{ }^{\circ} \mathrm{C}$ and $5 \% \mathrm{CO}_{2}$ and passaged three times a week using trypsin-EDTA for detachment.

For culture of ES14 cells the dishes were coated with $1 \%$ gelatine (Sigma, Henningsdorf) in PBS. The embryonic stem cells were cultured in BHK21 medium containing $1 \%$ FCS; $10 \%$ Knock-out Serum Replacement; 2 mM Lglutamine;0.1 mM non-essential amino acids; $1 \mathrm{mM}$ sodium pyruvate; $0.1 \mathrm{mM}$ $\beta$-mercaptoethanole (Sigma Henningsdorf) ; $1000 \mathrm{U} / \mathrm{ml}$ ESGRO. Knock-out Serum Replacement, non-essential amino acids and sodium pyruvate were purchased from Gibco, Carlsbad USA. ESGRO leukemia inhibitory factor was manufactured by Chemicon, Temecula USA.

ES14 cells were passaged three times a week and detached by trypsin-EDTA.

\subsubsection{Neuronal differentiation of cells in-vitro}

P19 embryo carcinoma cells can be in-vitro differentiated into postmitotic neurons by retinoic acid induction according to a protocol from $\mathrm{G}$. Banker et al. P19 cells were harvested from a confluent dish by trypsinization. $5 \times 10^{5}$ cells were seeded in $5 \mathrm{ml} \alpha \mathrm{MEM}$; 5 \% FCS; $1 \%$ PenStrep into a $5 \mathrm{~cm}$ non-adhesive biological Petri-Dish (Greiner, Frickenhausen). Retinoic acid (Sigma, Henningsdorf) from a $1 \mathrm{mM}$ stock solution in DMSO was added to a final concentration of $5 \times 10^{-7} \mathrm{M}$. For changing the medium after two days cell aggregates were collected in $15 \mathrm{ml}$ Falcon tube and the old medium was aspirated off. New medium and fresh retinoic acid were added. The cells were maintained as aggregates for another two days and harvested by sedimentation. Cell aggregates were washed with $5 \mathrm{ml}$ PBS and incubated in 2 $\mathrm{ml}$ trypsin-EDTA for $15 \mathrm{~min}$ at $37^{\circ} \mathrm{C}$. $2 \mathrm{ml}$ of $\alpha \mathrm{MEM} ; 5 \%$ FCS; $1 \%$ PenStrep were added and the cell suspension was passed 12 times through a fire polished pasteur pipet to yield a single cell suspension. Cells were plated at a density of $3.8 \times 10^{5}$ cells per $\mathrm{ml}$ for neuronal differentiation into a 6 -well plate with Matrigel-coated coverslips.

Fire-sterilized coverslips were coated with $10 \mu \mathrm{g} / \mathrm{ml}$ poly-L-lysine (Sigma, Henningsdorf) for $1 \mathrm{~h}$ at room temperature. The coverslips were washed with PBS and coated twice with Matrigel (bd biosciences, San Jose USA) diluted 1 : 250 in Optimem (Gibco, Carlsbad USA).

For neuronal differentiation P19 cells were maintained for up to 8 days in aMEM; 5 \% FCS; 1 \% PenStrep. A medium change was performed $24 \mathrm{~h}$ after plating and again after $48 \mathrm{~h}$.

Mouse embryonic stem cells can be differentiated into postmitotic neurons by co-culturing them on a monolayer of PA6 cells according to a protocol of Kawasaki et al.

ES cells from a confluent dish were collected by trypsinization and 500 cells / $\mathrm{ml}$ were seeded in differentiation medium consisting of BHK21 medium; $10 \%$ 
Knock-out Serum Replacement; $2 \mathrm{mM}$ L-glutamine;0.1 mM non-essential amino acids; $1 \mathrm{mM}$ sodium pyruvate; $0.1 \mathrm{mM} \beta$-mercaptoethanole on a monolayer of PA6 cells in a $10 \mathrm{~cm}$ cell culture dish. A medium change was performed on day 4 and every of the following 4 days. The ES cell derived neurons were cultured on the PA6 monolayer for another 6 days in differentiation medium supplied with N2 (Gibco, Carlsbad USA); $100 \mu \mathrm{M}$ tetrahydrobiopterin (Sigma, Henningsdorf) and $200 \mu \mathrm{M}$ ascorbate (Sigma, Henningsdorf).

\subsubsection{Transient Transfection of mammalian cells}

Mammalian cell lines were transfected with plasmid DNA using Lipofectamine 2000 reagent (Invitrogen, Karlsruhe) according to the manufacturer's instructions. For transfection of a $3.5 \mathrm{~cm}$ dish with confluent cells $2 \mu \mathrm{g}$ of plasmid DNA and $4 \mu \mathrm{l}$ of Lipofectamine 2000 reagent were diluted in $250 \mu \mathrm{l}$ Optimem (Gibco, Carlsbad USA) each and incubated for $5 \mathrm{~min}$ at room temperature. The DNA and the Lipofectamine 2000 dilutions were mixed and incubated for another $30 \mathrm{~min}$ at room temperature. $2 \mathrm{ml}$ of Optimem were added to the cells and overlaid with the transfection mixture. The transfection was carried out in a $37^{\circ} \mathrm{C}$ cell culture incubator with $5 \% \mathrm{CO}_{2}$ for $4 \mathrm{~h}$.

The transfection mixture was replaced by cell culture medium and the cells were assayed $24 \mathrm{~h}$ up to $72 \mathrm{~h}$ after the transfection.

Cell lines were transfected with plasmid DNA using the $\mathrm{CaPO}_{4}$ mediated ProFection Mammalian Transfection system purchased by Promega, Madison USA according to the manufacturer's instructions. Cells in a $60 \%$ confluent 10 $\mathrm{cm}$ dish were transfected with $20 \mu \mathrm{g}$ of plasmid DNA. In one tube the DNA was mixed with $2 \mathrm{M} \mathrm{CaCl}_{2}$ and sterile water to a final volume of $500 \mu \mathrm{l}$. The mixture was added dropwise by vortexing into a second tube containing $500 \mu$ l of $2 x$ HBS. The transfection mixture was incubated for $30 \mathrm{~min}$ at room temperature and added to the cells.

After incubation of the cells for $4 \mathrm{~h}$ or overnight in $37{ }^{\circ} \mathrm{C}$ incubator the transfection mixture was replaced by growth medium. The cells were assayed by western blotting $48 \mathrm{~h}$ to $72 \mathrm{~h}$ after the transfection

Mammalian cells were transfected with siRNA against $\mu 2$-adaptin by using Oligofectamine reagent (Invitrogen, Karlsruhe) according to the manufacturer's instructions.

Cells in a $50 \%$ confluent $3.5 \mathrm{~cm}$ dish were transfected with $2 \mu 10 \mu \mathrm{M}$ siRNA and $3 \mu$ Lipofectamine 2000 reagent. The siRNA and the Lipofectamine reagent were each diluted in $200 \mu \mathrm{l}$ Optimem and incubated at room temperature for 5 min. siRNA and Lipofectamine dilutions were mixed and incubated for $30 \mathrm{~min}$ at room temperature. The cells were overlaid with $800 \mu \mathrm{l}$ serum free medium and $200 \mu \mathrm{l}$ transfection mixture. After incubation for $4 \mathrm{~h}$ at $37^{\circ} \mathrm{C}$ and $5 \% \mathrm{CO}_{2} 500 \mu \mathrm{l}$ of growth medium containing $30 \%$ FCS were added. For co-transfection experiments cells were first transfected with $1 \mu \mathrm{g}$ of plasmid DNA and $2 \mu \mathrm{l}$ of Lipofectamine according to the protocol described above for $2 \mathrm{~h}$ at $37{ }^{\circ} \mathrm{C}$. A second transfection step including $2 \mu \mathrm{l}$ of $10 \mu \mathrm{M}$ siRNA, $1 \mu \mathrm{g}$ of plasmid DNA and $3 \mu \mathrm{l}$ Lipofectamine 2000 was carried out for an additional $2 \mathrm{~h}$. $24 \mathrm{~h}$ after siRNA transfection the cells were passaged on poly-L-lysine coated coverslips and assayed for the knock down of $\mu 2$-adaptin at day 3 after transfection. 


\subsubsection{Endocytosis assays in mammalian cells}

To assay endocytosis cells were starved for 45 min to $3 \mathrm{~h}$ in Optimem and surface labeled with $10 \mu \mathrm{g} / \mathrm{ml}$ Alexa488 conjugated transferrin (Molecular Probes, Leiden, Netherlands) in Optimem for 60 min at $4{ }^{\circ} \mathrm{C}$.

The incubation temperature was shifted to $37^{\circ} \mathrm{C}$ for $5 \mathrm{~min}$ up to $60 \mathrm{~min}$ to allow internalization of the transferrin. The cells were washed twice with $4{ }^{\circ} \mathrm{C} 0.1 \mathrm{M}$ $\mathrm{NaAc} ; 0.5 \mathrm{M} \mathrm{NaCl}$ to remove transferrin which was not internalized from the cell surface and fixed for 20 min with $4 \%$ paraformaldehyde (PFA) at room temperature.

For analyzing endocytosis of FLAG-tagged synaptotagmin constructs in transfected Cos 7 cells surface protein was labelled with $\alpha-F L A G$ monoclonal antibody M2 (Sigma, Henningsdorf) diluted 1:500 in Optimem. Internalization of the antibody was allowed for $30 \mathrm{~min}$ to $60 \mathrm{~min}$ by incubation at $37^{\circ} \mathrm{C}$. Cells were washed with PBS and fixed for 20 min with $4 \%$ PFA at room temperature. For analyzing endocytosis of HA-tagged GluR2 constructs in transfected Cos7 cells (Ahmadian et al.) surface proteins were labelled with $10 \mu \mathrm{g} / \mathrm{ml} \alpha-\mathrm{HA}$ monoclonal antibody F7 (Santa Cruz, Santa Cruz USA) in Optimem for 60 min at $4{ }^{\circ} \mathrm{C}$. Internalization of the antibody was allowed for $30 \mathrm{~min}$ to $60 \mathrm{~min}$ by incubation at $37^{\circ} \mathrm{C}$. To stimulate antibody-uptake cells were treated with $0.5 \mu \mathrm{M}$ insulin (Sigma, Henningsdorf) in $37^{\circ} \mathrm{C}$ Optimem for $10 \mathrm{~min}$ and incubated at 37 ${ }^{\circ} \mathrm{C}$ in Optimem without insulin for additional $50 \mathrm{~min}$. Cells were washed with PBS and fixed for 20 min with $4 \%$ PFA at room temperature.

For assaying endocytosis of endogenous synaptotagmin 1 in P19 neurons (Kraszewski et al., 1995) cells surface protein was labelled with $\alpha$ synaptotagmin lumenal domain antibody (Synaptic Systems; Göttingen). P19 neurons were washed once with Krebs-Ringer-HEPES solution $(28 \mathrm{mM} \mathrm{NaCl}$; $25 \mathrm{mM}$ HEPES pH 7.4; $4.8 \mathrm{mM} \mathrm{KCl} ; 1.3 \mathrm{mM} \mathrm{CaCl}_{2} ; 1.2 \mathrm{mM} \mathrm{MgSO}_{4} ; 1.2 \mathrm{mM}$ $\mathrm{KH}_{2} \mathrm{PO}_{4} / \mathrm{K}_{2} \mathrm{HPO}_{4} ; 5.6 \%$ glucose).

Cells were incubated with $\alpha$-synaptotagmin 1 antibody diluted $1: 100$ in KrebsRinger-HEPES high $\mathrm{K}^{+}(128 \mathrm{mM} \mathrm{NaCl} ; 25 \mathrm{mM}$ HEPES pH 7.4; $4.8 \mathrm{mM} \mathrm{KCl} ; 110$ $\mathrm{mM} \mathrm{CaCl}_{2} ; 1.2 \mathrm{mM} \mathrm{MgSO}_{4} ; 1.2 \mathrm{mM} \mathrm{KH}_{2} \mathrm{PO}_{4} / \mathrm{K}_{2} \mathrm{HPO}_{4} ; 5.6 \%$ glucose) for 10 min at $37^{\circ} \mathrm{C}$ and washed three times with $37^{\circ} \mathrm{C}$ Krebs-Ringer-HEPES. P19 neurons were fixed for 30 min with $4 \%$ PFA at room temperature.

\subsubsection{Immunofluorescence analysis of fixed tissues}

For immunofluorescence analysis cells were cultured on coverslips coated with $10 \mu \mathrm{g} / \mathrm{ml}$ poly-L-lysine (Sigma, Henningsdorf). The cells were fixed with either $4 \%$ para-formaldehyde at room temperature for $10 \mathrm{~min}$ up to $60 \mathrm{~min}$ or with methanole for $5 \mathrm{~min}$ at $-20{ }^{\circ} \mathrm{C}$. The fixative was removed and cells were washed twice in $120 \mathrm{mM} \mathrm{NaP}_{\mathrm{i}}$ and high salt PBS $\left(20 \mathrm{mM} \mathrm{NaP}_{\mathrm{i}} ; 0.5 \mathrm{M} \mathrm{NaCl} ; 0.3\right.$ $\%$ TritonX-100). Cells were blocked for $30 \mathrm{~min}$ in goat serum dilution buffer GSDB (High salt PBS; 30 \% goat serum) and incubated with primary antibody diluted in GSDB for 90 min at room temperature. Three washes with high salt PBS were performed before incubation of the cells with secondary Alexa dye conjugated antibody diluted $1: 150$ in GSDB for one hour at room temperature. Cells were washed twice with high salt PBS and $120 \mathrm{mM} \mathrm{NaP}_{i}$ before mounting the coverslips with Immu-Mount reagent (Thermo, UIm) on glass slides. 
To analyze antibody-internalization assays the cells were first stained under non-permaebilizing conditions with Alexa594 conjugated secondary antibody diluted 1:150 in GSDB without TritonX-100 to label the plasma membrane expressed proteins.

Unlabelled cell surface epitopes were blocked by applying goat-anti mouse serum in a dilution of $1: 5$ for $2 \mathrm{~h}$ at room temperature. Cells were then permeabilized with GSDB and intracellular primary antibody was labelled with Alexa488 conjugated secondary antibody diluted $1: 150$ in GSDB.

Cells were either imaged using an Olympus $1 \times 70$ inverted fluorescence microscope equipped with a $60 x$ oil immersion objective (NA 1.4). Images were processed using analysis software (soft imaging systems, Münster). Laser scanning microscopy images were acquired using a Zeiss LSM 2 equipped with a $60 \times$ oil immersion objective and processed with Zeiss LSM software.

Deconvolution microscopy was performed with an inverse Zeiss Axiovert 200 fluorescence microscope equipped with a $63 \times$ oil immersion objective. Three images to eight images along the z-axis with a distance of $0.1 \mu \mathrm{m}$ were taken. The image stack was deconvolved using stallion software purchased from intelligent imaging systems, Göttingen. 


\section{Results}

\subsubsection{The AMPA receptor subunit GluR2 cytoplasmic tail binds directly to $\mu 2$-adaptin of the AP-2 adaptor complex}

A recent study (Lee et al. 2002) has revealed that AMPA receptor internalization from the postsynaptic plasma membrane is required to induce NMDA-stimulated longterm depression in primary hippocampal neurons. Work from several groups has shown that AMPA receptors (AMPARs) undergo dynamin-1dependent endocytosis from the plasma membrane upon agonist stimulation (Man et al., 2000; Lin et al., 2000; Lee et al., 2002). These findings indicate that AMPARs can be internalized via clathrin-mediated endocytosis. Biochemical experiments have demonstrated that the native clathrin-adaptor complex AP-2 is able to bind to the cytoplasmic tail of the AMPA receptor subunit GluR2 (Lee et al., 2002). A basic stretch within the GluR2 cytoplasmic tail that also mediates binding to NSF, an ATPase involved in SNARE disassembly, is critical for interaction with AP-2.

Dibasic sorting motifs, interacting with the $\mu 2$-subunit of the AP-2 adaptor complex, function in clathrin-mediated endocytosis of various receptors such as the $\alpha_{1 \mathrm{~B}}$ adrenergic receptor and the $\beta 3$ - and $\gamma 2$ subunits of the GABA receptor (Diviani et al., 2003; Kittler submitted).

At the presynaptic terminal the SVP synaptotagmin 1 harbours a dibasic sorting motif in its C2B domain that is recognized by $\mu 2$-adaptin (Chapman et al., 1998; Haucke et al., 2000; Grass et al., 2004).

These findings raise the question whether the dibasic sorting motif in the AMPA receptor cytoplasmic tail directly binds to $\mu 2$-adaptin and, thus, if NMDA-induced internalization of the AMPA receptor may bear mechanistic similarity to the clathrin-mediated endocytosis of the presynaptic vesicle protein synaptotagmin.

To find out which proteins involved in clathrin-mediated endocytosis interact with the immobilized AMPA receptor cytoplasmic tail we used a pulldown assay with GST-fusion proteins of the AMPA receptor subunit GluR2 cytoplasmic tail immobilized on glutathione agarose beads. Fusion proteins were then incubated with TritonX-100 extracted rat brain homogenate (fig. 4.1).

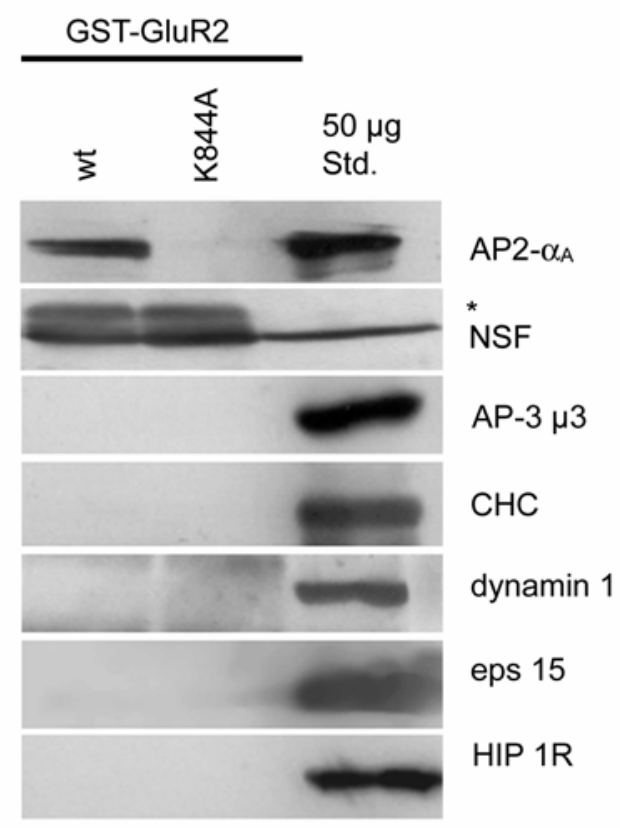

Fig. 4.1: AP-2 and NSF from ratbrain extract bind to GST-GluR2 cytoplasmic tail.

Tritonx-100 extracted ratbrain homogenate was for $1 \mathrm{~h}$ at $4{ }^{\circ} \mathrm{C}$ incubated with $100 \mu \mathrm{g}$ GST-GluR2 or GST-GluR2 K844A immobilized on glutathione sepharose beads. Samples were washed, bound proteins eluted and analyzed by SDSPAGE and immunoblotting using antibodies directed against AP- $2 \alpha$, NSF, AP-3 $\mu 3$, clathrin-heavy chain, dynamin 1 , eps 15 and HIP1R. The amino acid sequence basic AP-2 recognition motif within the GluR2 cytoplasmic tail is given below. The lysine mutated to alanine in GluR2 K844A is labelled in red. 
Bound proteins were analyzed by SDS-PAGE and immunoblotting.

The AP-2 adaptor complex bound only to the GluR2 wildtype tail; a mutant in carrying an alanine instead of a lysine (K844) was not capable of AP-2 binding anymore. This indicates that this basic residue is critical for the interaction between the AMPA receptor cytoplasmic tail and the AP-2 complex.

NSF also bound to the GluR2 wildtype cytoplasmic tail in agreement with ealier data. Mutation of the basic residue did not affect this interaction suggesting that lysine 844 is not involved in NSF binding to GluR2.

The AP-3 adaptor complex which is thought to function in transport of plasma membrane proteins to lysosomes (Odorizzi et al. 1998) was not pulled down from rat brain extract. The same holds true for clathrin the major coat protein recruited by AP-2. The GTPase dynamin that pinches off clathrin-coated vesicles from the plasma membrane (reviewed in Schafer 2004; Praefcke \& McMahon 2004), and eps15, an accesory protein for clathrin-mediated endocytosis also showed no binding to the AMPA receptor cytoplasmic tail.

In primary hippocampal neurons HIP1 co-localized with the AMPA receptor subunit GluR1 and HIP $\%$ mice show a defect in GluR1 containing AMPA receptor internalization which indicates that HIP1 might regulate AMPA receptor trafficking in-vivo (Metzler et al. 2003). In contrast to these findings HIP1R was not pulled down from rat brain extract by GST-GluR2 wildtype cytoplasmic tail.

Pulldown assays using detergent extracted rat brain homogenate do not give evidence if there is a direct interaction between AP-2 and the GluR2 cytoplasmic tail or if the AP-2 complex is co-purified with other unidentified proteins that directly bind to the GluR2 cytoplasmic tail.

Therefore. To address the questions if there is direct interaction between the GluR2 cytoplasmic tail and the AP-2 complex and to which subunit of the heterotetrameric complex binding occurs all four AP-2 subunits $\alpha_{c^{-}}, \beta$. -, $\mu 2-$ and $\sigma 2$-adaptins synthesized by coupled transcription / translation in-vitro were used in a pulldown assay with GST-GluR2 wildtype and K844A tails (fig. 4.2). Binding of the in-vitro-transcribed and translated proteins was resolved a SDS-PAGE and autoradiography of the dried and Comassie stained gel.

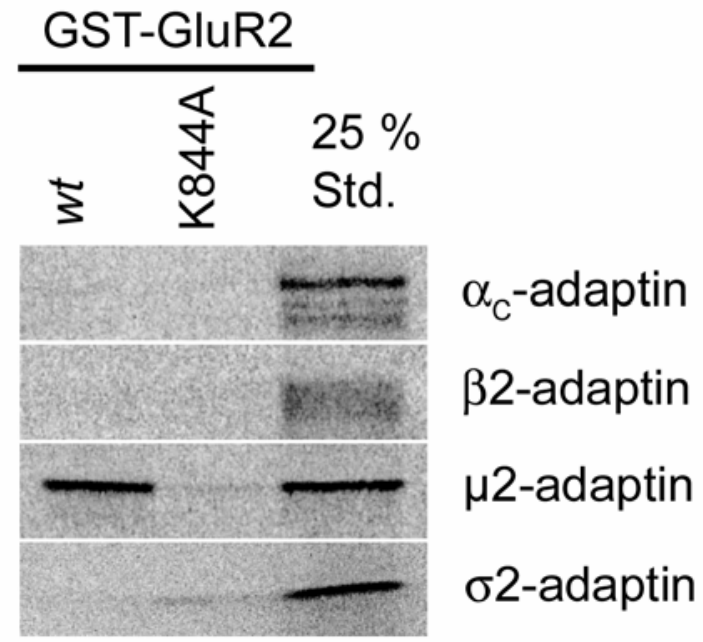

Fig. 4.2: The GluR2 cytoplasmic directly associates with $\mu 2$ adaptin. Recombinantly expressed GST-GluR2 wildtype and K844A cytoplasmic tail $(100 \mu \mathrm{g})$ coupled to glutathione agarose beads were incubated for $1 \mathrm{~h}$ at $4{ }^{\circ} \mathrm{C}$ with in-vitro transcribed / translated ${ }^{35}$ S-labeled AP-2 adaptor subunits. Samples were washed, affinity-purified material was resolved by SDSPAGE, and analyzed by autoradiography. 
GST-GluR2 cytoplasmic tail bound directly with the medium $\mu 2$-subunit of the AP-2 adaptor complex. A basic residue in the GluR2 tail is critical for this interaction because the GST-GluR2 K844A was unable to bind to $\mu 2$. No binding to the other AP-2 subunits $\alpha_{\mathrm{C}^{-}}, \beta 2$ 2- or $\sigma 2$-adaptin to GST-GluR2 was detected demonstrating that binding of GluR2 to the AP-2 complex is mediated via $\mu 2$.

To further confirm this result the pulldown of native AP-2 from rat brain extract by GST-GluR2 cytoplasmic tail was analyzed in the presence of increasing concentration of purified $\mu 2(158-435)$ as a competitor (Rohde et al. 2002) purified from E. coli (Fig. 4.3). The interaction was analyzed by SDS-PAGE and Western Blotting.

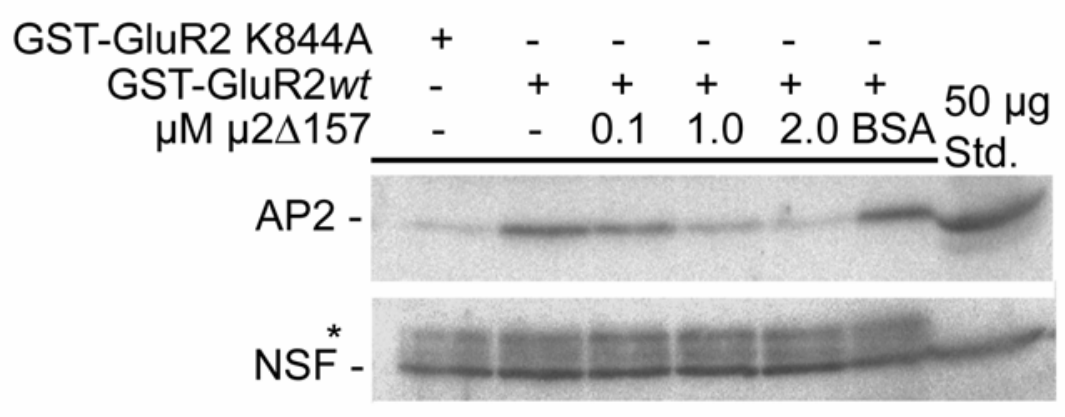

Fig. 4.3: Recombinant soluble $\mu 2 \Delta 157$ can compete the pulldown of native AP-2 complex from ratbrain extract by GST-GluR2. His $s_{6}$-tagged $\mu 2 \Delta 157$ was expressed and purified from $E$. coli. $100 \mu \mathrm{g}$ GST-GluR2 wildtype and K844 cytoplasmic tails immobilized on glutathione agarose beads were incubated for $1 \mathrm{~h}$ at $4{ }^{\circ} \mathrm{C}$ with TritonX-100 extracted ratbrain homogenate in presence of the given amounts of recombinant soluble $\mu 2 \Delta 157$ or BSA. Affinity purification was analyzed by SDS-PAGE and immunoblotting for AP- $2 \mu 2$ and NSF. The asterisk marks a band which is unspecifically recognized by the polyclonal NSF antibody.

A monoclonal antibody raised against an epitope within the first 157 amino acids of $\mu 2$-adaptin was unable to see recombinant $\mu 2 \Delta 157$ and was therefore used to detect native AP-2 from ratbrain extract. Binding of AP-2 to the AMPA receptor decreased in a dose-dependent manner upon increasing concentrations of $\mu 2 \Delta 157$. In contrast, NSF interaction with GST-GluR2 cytoplasmic tail was not affected by $\mu 2 \Delta 157$.

Taken together these data provide strong evidence for direct binding of the AMPA receptor subunit GluR2 to $\mu 2$-adaptin, the medium subunit of the clathrinadaptor complex AP-2 in-vitro. This is critically dependent on a lysine at position 844 in the AMPA-receptor cytoplasmic tail. 


\subsubsection{A basic sorting motif in the AMPA receptor cytoplasmic tail mediates direct interaction with $\mu 2$-adaptin}

A sequence alignment of different AMPA receptor subunits reveals that the cytoplasmic tails of GluR1 and GluR3 contain a basic stretch similar to that in GluR2 (fig. 4.4). In pulldown assays native AP-2 complex from ratbrain interacted with the GluR1 and GluR3 cytoplasmic tails immobilized on glutathione agarose (Lee et al. 2002). We were interested in the question if these subunits can also directly interact with $\mu 2$-adaptin. Therefore GST-fusion proteins of the GluR1, GluR2 and GluR3 cytoplasmic tails immonbilized on glutathione sepharose were incubated with recombinantly expressed soluble $\mu 2 \Delta 157$ purified from $\mathrm{E}$. coli and binding was analyzed by SDS-PAGE and Comassie staining (fig. 4.5).

$\begin{array}{lll}\text { GluR1 } & \text { cytoplasmic tail } & \text { KRMKGFCLIP } \\ \text { GluR2 } & \text { cytoplasmic tail } & \text { KRMKVAKNPQ } \\ \text { GluR3 cytoplasmic tail } & \text { KRMKLTCLTP }\end{array}$

Fig. 4.4: Sequence alignment of basic stretches in the AMPA receptor subunits GluR1, GluR2 and GluR3. Basic residues are labeled in red.

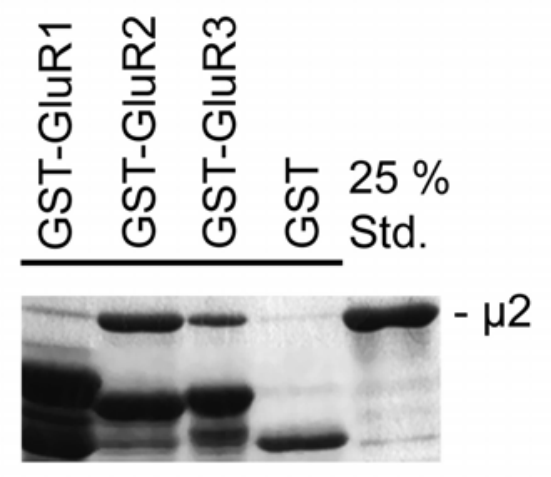

Fig. 4.5: $\mu 2 \Delta 157$ directly binds to the cytoplasmic tails of different AMPA receptor subunits GluR1, GluR2 and GluR3. $20 \mu \mathrm{g}$ GST-fusion proteins of GluR1, GluR2 and GluR3 cytoplasmic tails immobilized on glutathione sepharose beads were incubated for $1 \mathrm{~h}$ at $4{ }^{\circ} \mathrm{C}$ with recombinantly expressed soluble $\mu 2 \Delta 157$. Binding of $\mu 2$ was detected by SDS-PAGE and Comassie Blue staining.

All three AMPA receptor subunit cytoplasmic tails bound directly to $\mu 2$-adaptin. GluR2 showed the strongest interaction with $\mu 2$ in comparison to GluR1 and GluR3.

Therefore we used the GluR2 cytoplasmic tail as a representative for other AMPA receptor subunits in further biochemical experiments to study binding to AP-2.

The minimal binding motif for NSF with the GluR2 cytoplasmic tail comprises amino acids K844 to Q853 (Nishimune et al. 1998). Deletion of the amino acids A849 and Q853 within this sequence abolished binding of NSF but showed no effect on AP-2 binding, suggesting that different residues are responsible for the association with these two proteins. 
By contrast exchange of the basic residues K844, R845 or K847 to alanine within this region impaired the association of native AP-2 from rat brain extracts (Lee et al. 2002).

To find out whether reduced pulldown of AP-2 was due to an impaired interaction of the GluR2 cytoplasmic tail point mutants (fig.4.6) with $\mu 2$ we performed a pulldown assay using soluble $\mu 2 \Delta 157$ (fig. 4.7).

GluR2 cytoplasmic tail

${ }^{834}$ EFCYKSRAEA KRMKVAKNPQ NTNPSSSQNS QNFATYKECY NVYGIESVKL ${ }^{883}$

Fig. 4.6: Sequence of the AMPA receptor subunit GluR2 cytoplasmic tail. Mutated residues are labeled red and the NSF / AP-2 binding site is written bold.

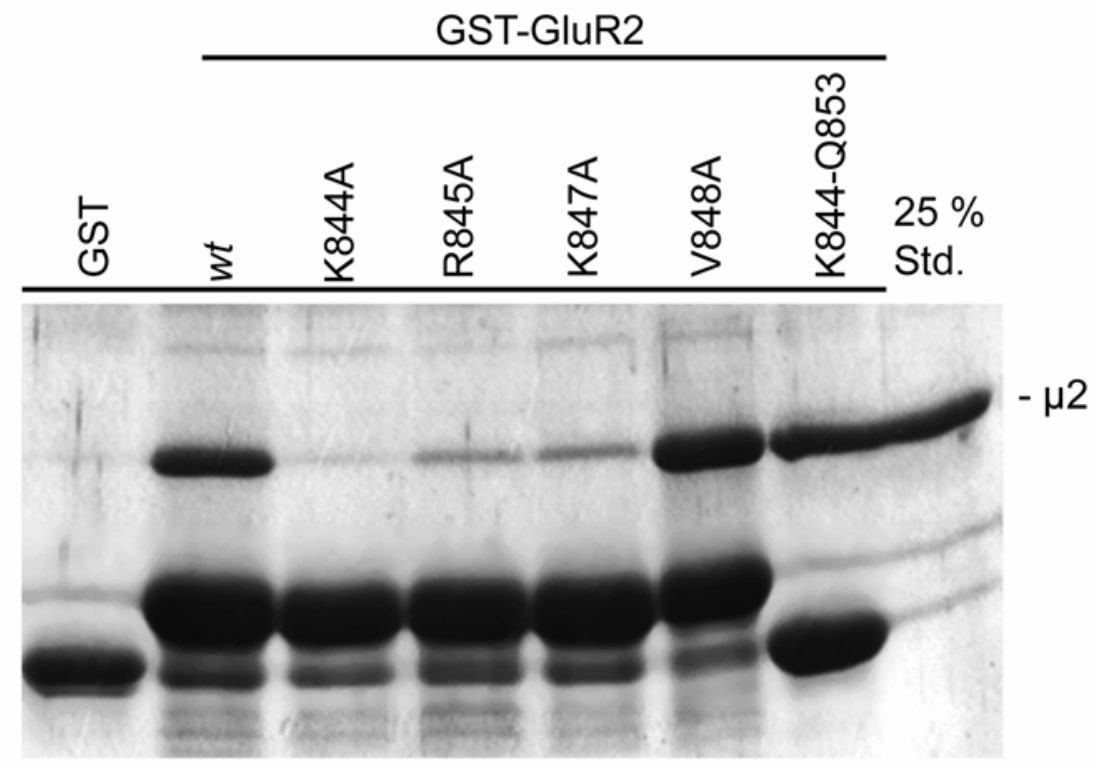

Fig. 4.7: Binding of $\mu 2$ to GluR2 depends on three basic residues K844, R845 and K847 in the GluR2 cytoplasmic tail. Amino acids K844, R835, K847 and V848 within the GluR2 cytoplasmic tail were mutated into alanines by site directed mutagenesis. $20 \mu \mathrm{g}$ of GST, GSTGluR2 wildtype or point mutants immobilized on glutathione sepharose were incubated for $1 \mathrm{~h}$ at $4{ }^{\circ} \mathrm{C}$ with soluble $\mu 2 \Delta 157$. Binding of $\mu 2$ was analyzed by SDS-PAGE and Comassie Blue staining.

Exchange of three basic residues $\mathrm{K} 844$, R845 or K847 to alanines almost abolished binding of $\mu 2$ to the GluR2 cytoplasmic tail whereas mutation of a valine at position 848 showed no effect on the interaction. Interestingly, a GSTfusion peptide encompassing the NSF binding site from K844 to Q853 in the GluR cytoplasmic tail was sufficient to mediate binding of $\mu 2$-adaptin. All three basic residues required for GluR2 interaction with $\mu 2$ were located within this peptide.

This finding demonstrates that interaction between $\mu 2$ and GluR2 is critically dependent on a number of basic residues within the GluR2 cytoplasmic tail and that mutation of any of them disrupts binding. These three residues K844, R845 and K847 are located within the membrane proximal region of the GluR2 cytoplasmic tail, which also harbours an overlapping, yet distinct, binding site for NSF. 


\subsubsection{Proteins from the pre- and postsynaptic site interact with $\mu 2-a d a p t i n$ via a basic sorting motif}

Basic sorting motifs that are recognized by the $\mu 2$-subunit of the clathrin-adaptor AP-2 have been recently characterized in various proteins (see fig. 4.8):

$\begin{array}{ll}\text { Synaptotagmin 1 } & \text { pep KR } \\ \text { Synaptotagmin 1 } & \text { pep AA } \\ \text { AMPA receptor GluR2 } & \text { pep2r } \\ \text { GABA receptor } \beta 3 & \end{array}$

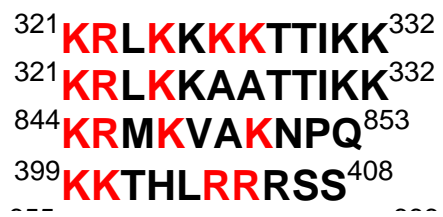

Fig. 4.8: Alignment of basic sorting motifs. Basic residues critical for recognition by the AP-2 complex are labelled in red. The corresponding synthetic peptides are indicated. The peptides pepKR and pepAA comprise the synaptotagmin 1 basic sorting motif or a mutant motif. pep2r comprises the GluR2 basic sorting motif.

Studies from Diviani et al. (2003) have demonstrated that the G-protein coupled $\alpha_{1 B}$-adrenergic receptor associates directly with $\mu 2$-adaptin via a stretch of eight arginines. A dibasic sorting motif within the $\mathrm{C} 2 \mathrm{~B}$ domain of the synaptic vesicle protein synaptotagmin 1 mediates direct interaction with $\mu 2$-adaptin and is sufficient to target internalization of a multimeric chimeric fusion protein (Chapman et al., 1998; Haucke et al., 2000; Grass et al., 2004). AP-2 has also been shown to be necessary for internalization of the postsynaptic inhibitory $\mathrm{GABA}_{\mathrm{A}}$ receptor (Kittler et al., submitted). The $\mathrm{GABA}_{A}$ receptor subunits $\beta 3$ and $\gamma 2$ bind to $\mu 2$ via a basic sorting motif between their third and forth transmembrane domain (Kittler et al., unpublished data). These findings suggest that basic sorting motifs that may interact with $\mu 2$-adaptin have a role in the regulated uptake of proteins from both sides of the synapse. To test this hypothesis we analyzed if basic sorting motifs derived from these proteins were able to compete for each other with regard to AP-2 binding.

Therefore synthetic peptides comprising basic sorting motifs derived from synaptotagmin 1 (pepKR) and GluR2 (pep2r) were used in pulldown assays and incubated with GST-GluR2 immobilized on glutathione sepharose and TritonX100 extracted rat brain homogenate or recombinant soluble $\mu 2 \Delta 157$.

First, we addressed the question if direct association between GST-GluR2 and $\mu 2 \Delta 157$ can be competed by a synaptotagmin 1 peptide (pepKR) or peptides encompassing other classical endocytic sorting motifs such as tyrosine- (YQRL) or dileucine- (LL) based motifs. GST-GluR2 was incubated with recombinant $\mu 2 \Delta 157$ in the presence of different synthetic peptides. Binding was analyzed by SDS-PAGE and Comassie staining. The basic synaptotagmin 1 peptide (pepKR) completely inhibited the interaction between GST-GluR2 and $\mu 2$ whereas neither a tyrosine- motif peptide (YQRL), a mutated tyrosine-motif peptide (AQRL), nor a dileucine-motif peptide (LL) interfered with binding of $\mu 2$ to GST-GluR2 (fig.4.9). 


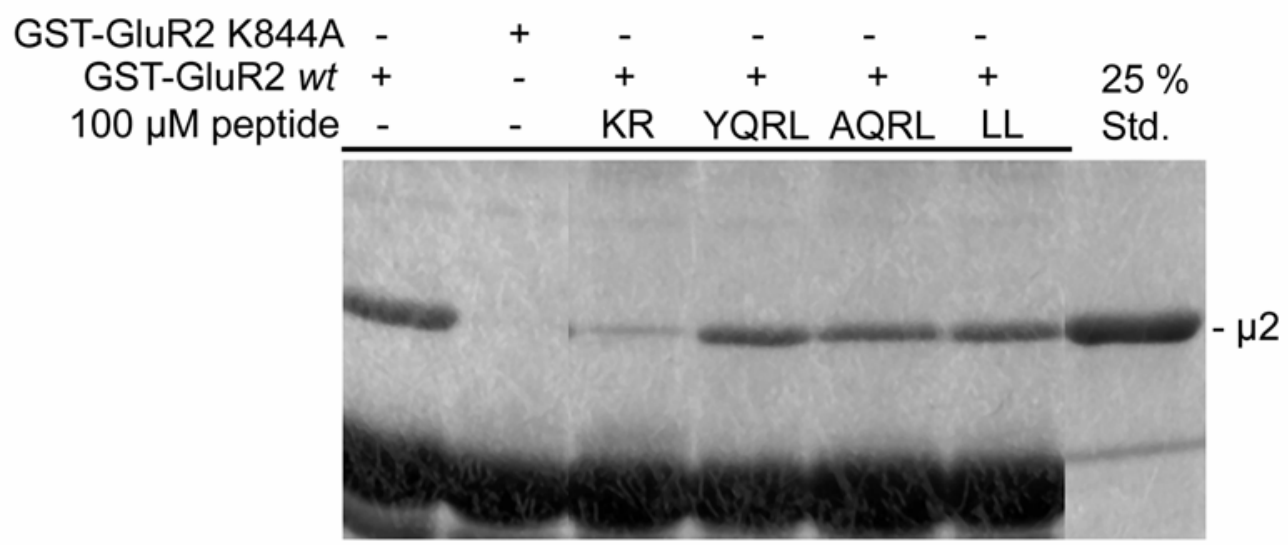

Fig. 4.9 : The interaction between GluR2 and $\mu 2$ can be competed by a basic peptide comprising the synatotagmin 1 binding site for $\mu 2$-adaptin. $20 \mu \mathrm{g}$ GST-GluR2 wildtype or K844A cytoplasmic tail were incubated for $1 \mathrm{~h}$ at $4{ }^{\circ} \mathrm{C}$ with soluble $\mu 2 \Delta 157$ in presence of 100 $\mu \mathrm{M}$ synthetic peptides derived from synaptotagmin 1 (KR), a tyrosine-based sorting motif (YQRL), a mutated tyrosine-based sorting motif (AQRL) or a dileucine-based sorting motif (LL). Binding of $\mu 2$ was detected by SDS-PAGE and Comassie Blue staining.

By contrast, the association of native AP-2 complexes from rat brain extract by GST-GluR2 was effectively competed by basic peptides derived from the AMPA receptor and synaptotagmin 1 sorting motifs pep2r and pepKR (fig. 4.10).

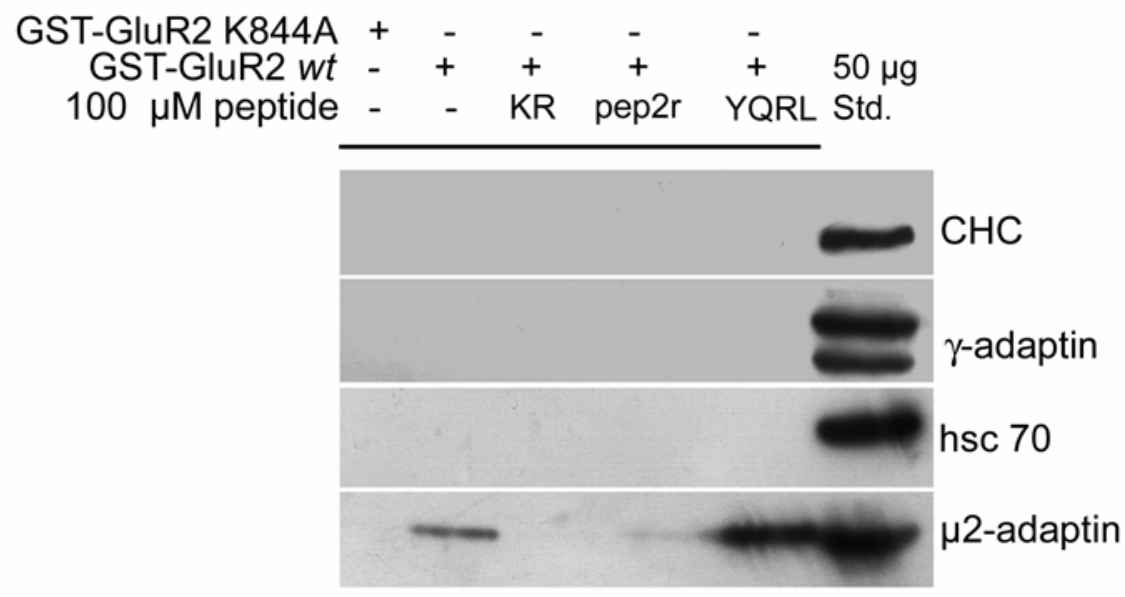

Fig. 4.10: Basic peptides derived from the AMPA receptor or synaptotagmin 1 basic sorting motifs compete the interaction between native AP-2 complex and the GluR2 cytoplasmic tail. TritonX-100 extracted rat brain was incubated for $1 \mathrm{~h}$ at $4{ }^{\circ} \mathrm{C}$ with $100 \mu \mathrm{g}$ GST-GluR2 wildtype or K844A cytoplasmic tail in presence of $100 \mu \mathrm{M}$ synthetic peptides derived from synaptotagmin $1(K R)$, the AMPA receptor pep2r (KRMKVAKNPQ) a tyrosine- based sorting motif (YQRL), a mutated tyrosine-based sorting motif (AQRL) or a dileucine- based sorting motif (LL). The pulldown was analyzed by SDS-PAGE and immunoblotting for AP-2 $\mu 2$, $\gamma$-adaptin, hsc 70 and clathrin heavy chain.

A tyrosine-peptide YQRL, a mutated tyrosine-peptide AQRL or a dileucinepeptide LL did not affect association between GluR2 and AP-2.

These results suggest that basic sorting motifs from pre- and postsynaptic proteins bind to $\mu 2$-adaptin by a similar mechanism and that these motifs can 
compete for each other. Since peptides encompassing other classical endocytosis motifs had no influence on binding of GluR2 to $\mu 2$ it seems that the binding site for basic sorting motifs at $\mu 2$-adaptin is distinct from that for tyrosine based sorting motifs within the $\mu 2$ subdomain A (Owen et al, 1999).

We aimed to confirm that competition of $\mu 2$ interacting with GluR2 is dependent on a dibasic motif within the synaptotagmin 1 derived peptide. Therefore a mutant synthetic peptide (pepAA) was used in pulldown assays with GST-GluR2 and recombinant $\mu 2 \Delta 157$. Comassie staining shows that binding of $\mu 2$ to GSTGluR2 decreased in a dose-dependent manner upon incubation with increasing concentrations of pepKR (fig. 4.11) In contrast the mutant peptide (pepAA) did not affect binding of $\mu 2$ to GluR2.

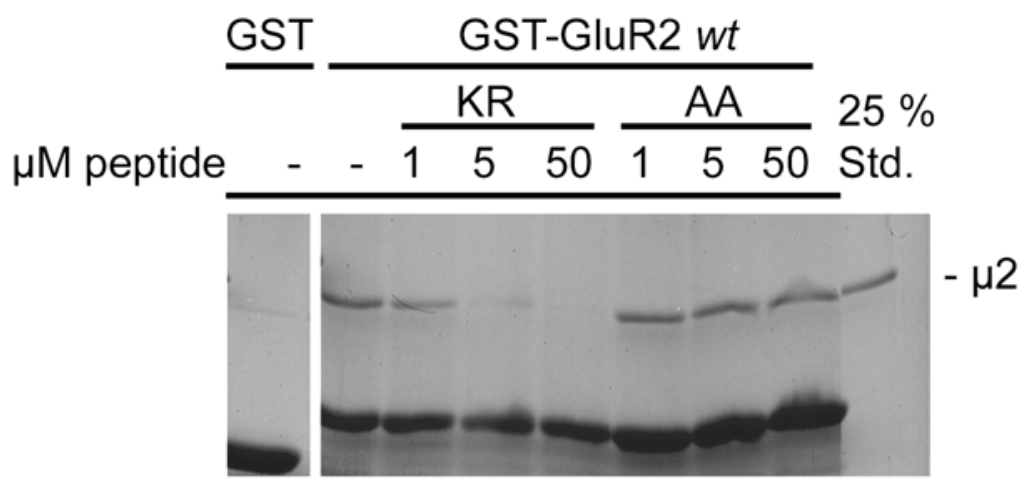

Fig. 4.11: Competition of the interaction between $\mu 2$ and GluR2 by a synaptotagmin 1 derived peptide (KR) is dependent on a dibasic motif. $20 \mu \mathrm{g}$ GST or GST-GluR2 were incubated for $1 \mathrm{~h}$ at $4{ }^{\circ} \mathrm{C}$ with soluble $\mu 2 \Delta 157$ in presence of the indicated amounts of a basic synaptotagmin 1 derived peptide (KR) or mutated peptide (AA). Affinity purification of $\mu 2 \Delta 157$ was detected by SDS-PAGE and Comassie Blue staining.

Pulldown of native AP-2 complex from ratbrain extract by GST-GluR2 was also dose dependently impaired by the synaptotagmin 1 wildtype peptide (KR) whereas the mutant peptide was unable to compete binding of AP-2 to GluR2 (fig. 4.12).

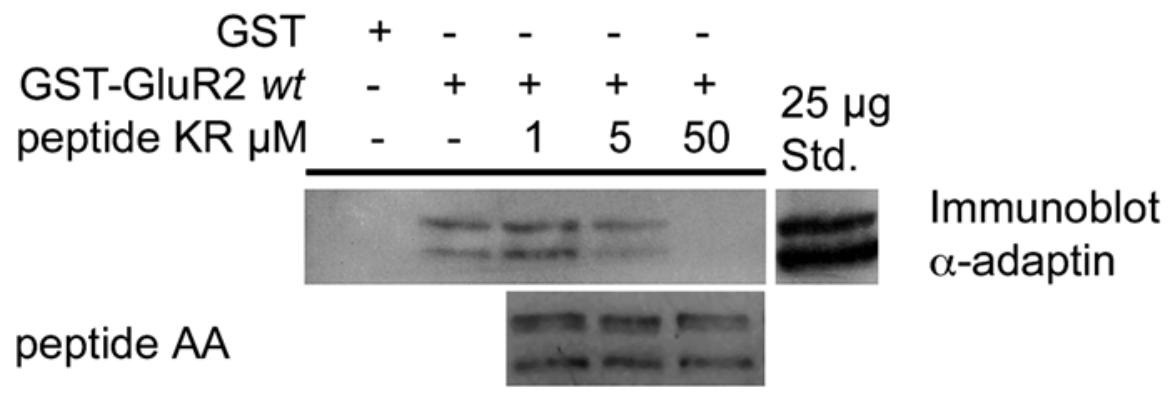

Fig. 4.12: Disruption of the pull down of native AP-2 complex by GluR2 depends on two basic residues within a synthetic synaptotagmin 1 derived peptide KR. TritonX-100 extracted rat brain was incubated for $1 \mathrm{~h}$ at $4{ }^{\circ} \mathrm{C}$ with $100 \mu \mathrm{g}$ GST or GST-GluR2 wildtype cytoplasmic tail in presence of the indicated concentrations of a synthetic peptide derived from synaptotagmin $1(K R)$ or a mutant peptide (AA). Samples were analyzed by SDS-PAGE and immunoblotting for AP-2 $\alpha$-adaptin. 
These results clearly show that a dibasic motif mediates recognition of the presynaptic protein synaptotagmin 1 or the postsynaptic AMPA receptor by the AP-2 subunit $\mu 2$.

Since the postsynaptic GABA receptor subunit $\gamma 2$ also binds to $\mu 2$-adaptin via a basic sorting motif, we were interested to see if the synaptotagmin 1 peptide KR was also able to overcome the interaction between $\mu 2$ and $\gamma 2$ cytoplasmic tail. Therefore a pulldown assay from rat brain extract using GST- $\gamma 2$ in presence of increasing concentrations of the basic peptide $(K R)$ or the mutant peptide (AA) was performed (Fig. 4.13).

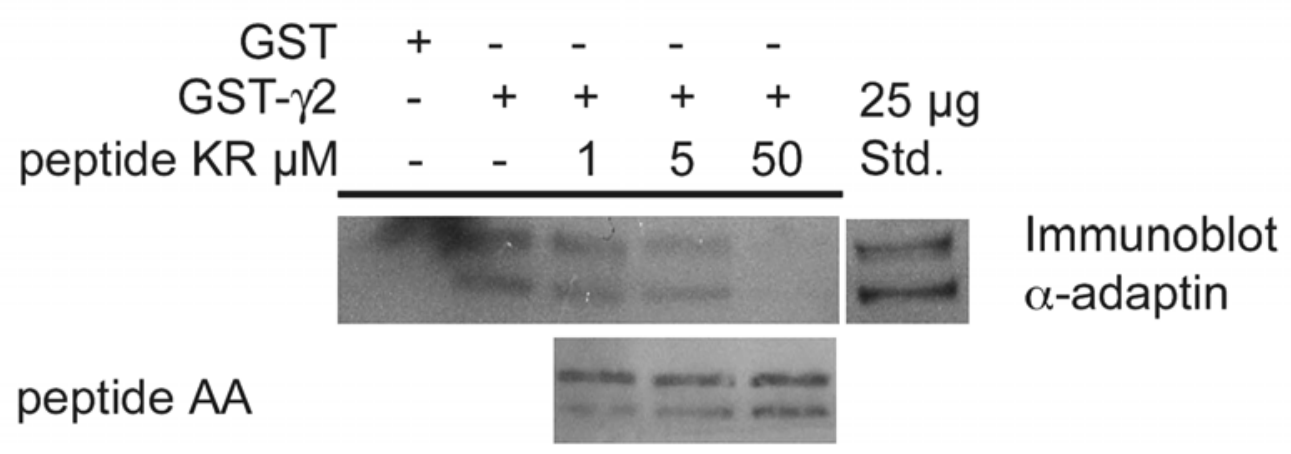

Fig. 4.13: Fig. 3C: Competition of the interaction between $\mu 2$ and the $\mathrm{GABA}_{A}$ receptor subunit $\gamma 2$ by a synaptotagmin 1 derived basic peptide (KR). TritonX-100 extracted ratbrain was incubated for $1 \mathrm{~h}$ at $4{ }^{\circ} \mathrm{C}$ with $100 \mu \mathrm{g} \mathrm{GST}$ or GST- $\gamma 2$ in presence of the indicated concentrations of a synthetic peptide derived from synaptotagmin $1(K R)$ or a mutant peptide (AA). Samples were analyzed by SDS-PAGE and immunoblotting for AP-2 $\alpha$-adaptin.

Western blot analysis revealed that analogous to previous experiments

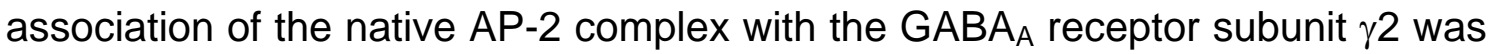
competed by the synaptotagmin 1 wildtype peptide (KR). The mutant peptide AA did not affect binding in a dose dependent manner demonstrating again the requirement for basic residues for the interaction between $\gamma 2$ and $\mu 2$-adaptin.

Taken together these findings imply a role for basic sorting motifs as linkers to the endocytic machinery in the ligand-regulated uptake of postsynaptic receptors such as the AMPA or GABA receptor and presynaptic proteins including synaptotagmin 1 . Interaction between $\mu 2$-adaptin and basic sorting motifs in pre- or postsynaptic proteins seems to occur via a similar mechanism because a peptide encompassing a sorting motif derived from presynaptic synaptotagmin 1 effectively competes binding of $\mu 2$ to postsynaptic receptor sorting motifs. A peptide comprising a mutation of the dibasic synaptotagmin 1 sorting motif was unable to disrupt binding of AP-2 showing that very few basic residues within a sorting motif are sufficient to mediate interaction with $\mu 2$ adaptin. It is thus reasonable to assume that basic sorting motifs exist in a variety of proteins and that they have a general function in regulated endocytosis of multimeric cargo membrane proteins from the plasma lemma in different physiological processes. 


\subsubsection{Characterization of a putative binding site for basic sorting motifs in $\mu 2$-adaptin}

Up to now only the recognition site for tyrosine based sorting motifs in $\mu 2$ adaptin has been characterized in detail by work of Owen and Evans (1999), where they co-crystallized $\mu 2 \Delta 157$ together with a tyrosine based synthetic peptide: $\mu 2$-adaptin consists of a flexible linker region (residues 1 to 158) which links the subunit to the AP-2 core. The C-terminal part (residues 158 to 435) has an elongated banana shaped structure composed of $\beta$-sheets that can be divided into two $\beta$-sandwich domains A (residues 158 to 282 and 394 to 435) and $B$ (residues 283 to 393 ).

The binding site for tyrosine based sorting motifs is located to the A domain of $\mu 2$. Interaction is mainly mediated by hydrophobic interactions between the tyrosine ring of the sorting motif and aromatic side chains in the binding pocket (Owen 1999).

In contrast to the interaction of tyrosine based sorting motifs with the A domain of $\mu 2$, work of Diviani et al. 2003 located the binding site for a motif of eight arginines in the $\alpha_{1 \mathrm{~B}}$ adrenergic receptor (AR) cytoplasmic tail to the $\mathrm{N}$-terminus and the $B$ domain of $\mu 2$-adaptin. In a pulldown experiment using GST-tagged immobilized AR tail and GFP-tagged $\mu 2$-fragments the $\mathrm{N}$-terminal portion (residue 1 to 157) and the $B$ domain of $\mu 2$ (residues 283 to 435 ) bound to the $\alpha_{1 \mathrm{~B}}$ adrenergic receptor (Diviani et al. 2003).

Based on these results we assumed that acidic residues on the surface of the $\mu 2 \mathrm{~B}$ domain would play a role in the recognition of basic sorting motifs.

Since tyrosine based synthetic peptides could not disrupt the interaction between GluR2 and $\mu 2$ in previous pulldown experiments (see section) we concluded that basic sorting motifs bind to a site in $\mu 2$ which is distinct from the recognition site for tyrosine based sorting motifs.

An alignment of $\mu 2$ with the $\mu$ subunits of other clathrin-adaptor complexes AP1, AP-3 and AP-4 revealed two acidic patches located at the surface of the $\mu 2 \mathrm{~B}$ domain of $\mu 2$ which were not conserved among other $\mu$-adaptins (see fig.4.14).

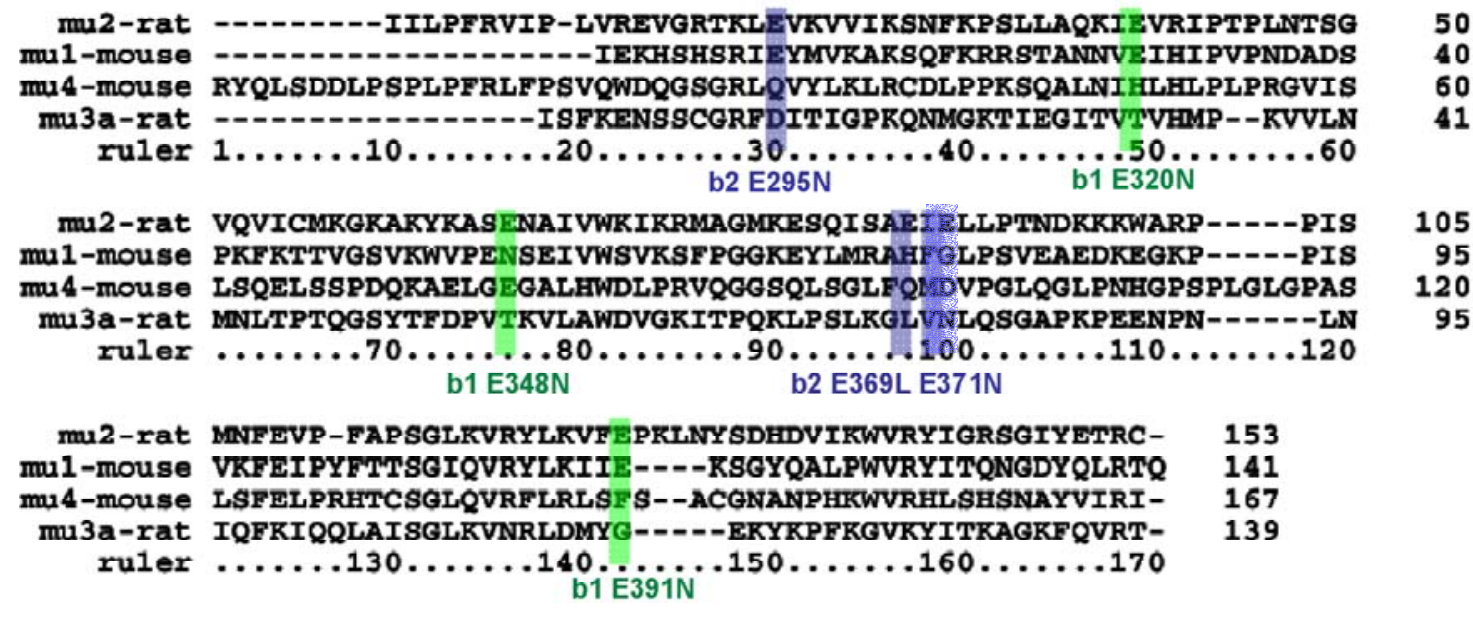

$\mathbf{t}$

Fig. 4.14: Protein sequence alignment of $\mu 2$-adaptin $B$ domain with $\mu 1-, \mu 3-$ and $\mu 4-$ adaptin. The putative binding site 1 (b1) for basic sorting motifs and the inserted amino acid exchanges E320N, E348N and E391N are labelled green. Mutations inserted in second putative binding site (b2) E320N, E369L and E371N are labelled blue. 
By site-directed mutagenesis of $\mu 2$ we addressed the question if these two acidic patches were putative recognition sites for the AMPA receptor basic sorting motif.

Two mutants were created: The mutant $\mu 2$ b1 contained the following exchanges of acidic surface residues E320N, E348N and E391K. In the second construct $\mu 2$ b2 the mutations E295N, E369L and E371N were inserted. To preserve structural integrity of the $\mu 2$ mutants we inserted non-acidic residues from other $\mu$-adaptins that align with acidic residues in $\mu 2$.

To address the question if the mutant $\mu 2$-adaptins were still able to interact with the GluR2 cytoplasmic tail HA-tagged full length $\mu 2 w t, \mu 2$ b1 and $\mu 2$ b2 were expressed in HEK 293 cells.

In a pulldown assay TritonX-100 extract of transfected HEK cells was incubated with GST and GST-GluR2 wt immobilized on glutathione sepharose. Binding of $\mu 2$ was analyzed by SDS-PAGE and immunoblotting for the HA-tag (Fig.4.15).

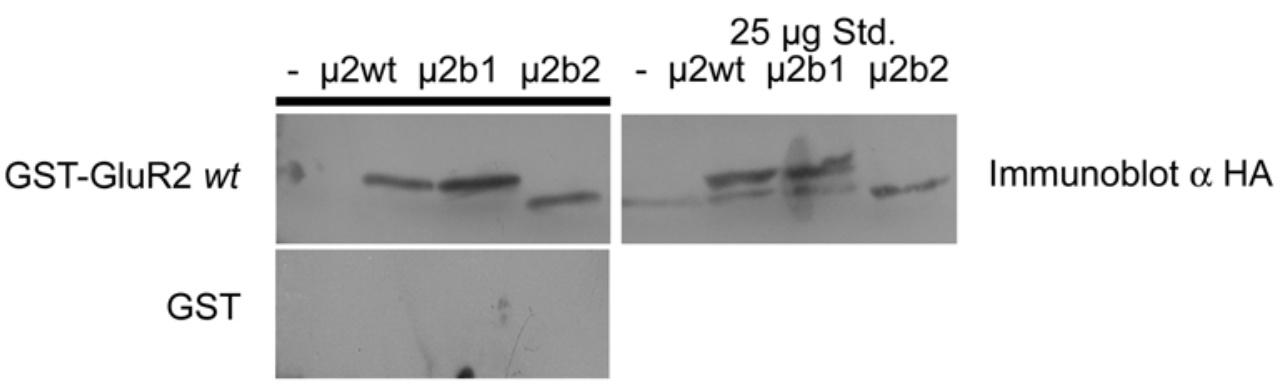

Fig. 4.15: Mutation of acidic patches on the $\mu 2$ surface does not disrupt binding to GluR2. HEK 293 cells were transfected with HA-tagged $\mu 2 w t, \mu 2$ b1 and $\mu 2$ b2. $500 \mu g$ Triton-X 100 extracted cells were incubated for $1 \mathrm{~h}$ at $4{ }^{\circ} \mathrm{C}$ with GST or GST-GluR2 immobilized on glutathione sepharose beads. Affinity purification was analyzed by SDS-PAGE and immunoblotting for the HA-tag.

Neither $\mu 2$ b1 nor $\mu 2$ b2 were impaired in binding to GST-GluR2. Both mutants interacted with GluR2 to the same degree as wildtype $\mu 2$. No binding to GST was detected.

Thus interaction between the AMPA receptor cytoplasmic tail and $\mu 2$-adaptin could not be disrupted by mutation of two acidic patches in the $\mu 2 \mathrm{~B}$ domain. The mutated acidic residues do not seem to be involved in the binding of basic sorting motifs.

These results suggest that instead of targeted mutation of putative binding sites a random mutagenesis of $\mu 2$-adaptin seems to be more promising for finding the interaction site for basic sorting motifs.

An alternative structural approach would be to co-crystallize $\mu 2$-adaptin together with a synthetic basic peptide according to the work of Owen and Ewans on tyrosine based sorting motifs. 


\subsubsection{Plasma membrane recruitment of soluble $\mu 2 \Delta 157$ by a synaptotagmin 1 chimera carrying the AMPA receptor basic sorting motif in transfected cells}

Our biochemical experiments described above have indicated that AMPA receptors interact with the $\mu 2$-subunit of the clathrin-adaptor AP-2 via a basic sorting motif in the membrane proximal portion of their cytoplasmic tails. A mutagenesis screen revealed that lysine and arginine at positions 844 and 845 are critical for binding to $\mu 2$-adaptin.

To confirm this interaction in an in-vivo model a chimeric approach was used. The synaptic vesicle protein synaptotagmin 1 harbours a basic sorting motif within its C2B domain that is recognized by $\mu 2$-adaptin (Chapman et al., 1998; Haucke et al., 2000; Grass et al., 2000). This motif was able to compete the interaction between AP-2 and the GluR2 cytoplasmic tail in in-vitro experiments. In a chimeric construct the AMPA receptor basic sorting motif pep2r or a mutant motif in which two basic residues were exchanged for alanines (pep2M) were used to replace the $\mathrm{C} 2 \mathrm{~B}$ domain of synaptotagmin 1(Fig. 4.16).

\section{\begin{tabular}{l|l|l|} 
FLAG & Syt $1 \triangle$ C2B & KRMKVAKNPQ \\
\hline
\end{tabular}}

Fig. 4.17: FLAG-Synaptotagmin 1 chimera carrying the AMPA receptor basic sorting motif. The $\mathrm{C} 2 \mathrm{~B}$ domain of synaptotagmin 1 that harbours a dibasic recognition site for $\mu 2$ adaptin was replaced by the AMPA receptor derived basic sorting motif to generate a FLAGtagged chimeric construct FLAG-Syt1 $\triangle$ C2Bpep2r. In a mutant construct FLAG-Syt1 $\triangle$ C2Bpep2M the lysin and argine labelled in red were exchanged for alanines.

The construct also carried an extracellular FLAG-tag for immunocytochemical labelling. Work of Feany et al. (1991) has shown that synaptotagmin 1 is sorted to the plasma membrane of fibroblasts. In agreement with this observation synaptotagmin 1 chimeric constructs FLAG-Syt1 $\Delta$ C2Bpep2 $r$ and pep2M were targeted to the plasma membrane of transfected HEK293 fibroblasts (Fig 4B). An HA-tagged $\mu 2$ construct lacking the first 157 residues $(\mathrm{HA}-\mu 2 \Delta 157)$ remained soluble in the cytoplasm and was not incorporated into the native AP-2 complex when expressed HEK293 cells (Fig. 4B) as expected from the crystal structure of the AP-2 core complex (Collins et al., 2002). HEK 293 cells were cotransfected with FLAG-Syt1 $\triangle$ C2Bpep2 $r$ carrying the AMPA receptor basic sorting motif and soluble $\mathrm{HA}-\mu 2 \Delta 157$ to address the question if the basic peptide was able to recruit $\mu 2$-adaptin to the plasma membrane of transfected cells. After fixation FLAG-Syt1 $\triangle$ C2Bpep2 $r$ (red) at the plasma membrane of transfected cells was immunocytochemically labelled under non-permeabilizing conditions. Cells were then permeabilized by TritonX-100 and stained against intracellular HA- $\mu 2 \Delta 157$ (green fluorescence) (fig. 4.18). The synaptotagmin 1 chimera carrying the AMPA receptor basic sorting motif FLAG-Syt1 $\Delta$ C2Bpep2 $r$ was able to recruit soluble $\mathrm{HA}-\mu 2 \Delta 157$ from the cytoplasm to the plasma membrane of transfected HEK 293 cells (fig. 4.18, upper panel). In contrast the mutant chimera FLAG-Syt1 $\triangle$ C2Bpep2r lacking two basic residues did not affect the cytoplasmic distribution of $\mathrm{HA}-\mu 2 \Delta 157$ (middle panel). A truncated synaptotamin 1 devoid of the C2B domain FLAG-Syt1 $\triangle \mathrm{C} 2 \mathrm{~B}$ was also unable to recruit $\mu 2$-adaptin to the plasma membrane (lower panel). 
Single transfections:
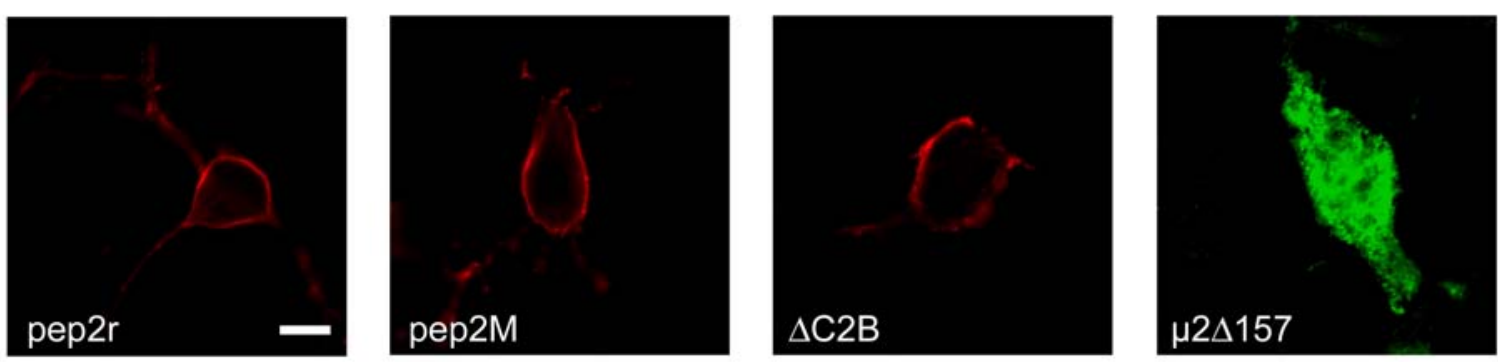

\section{Co-transfections:}
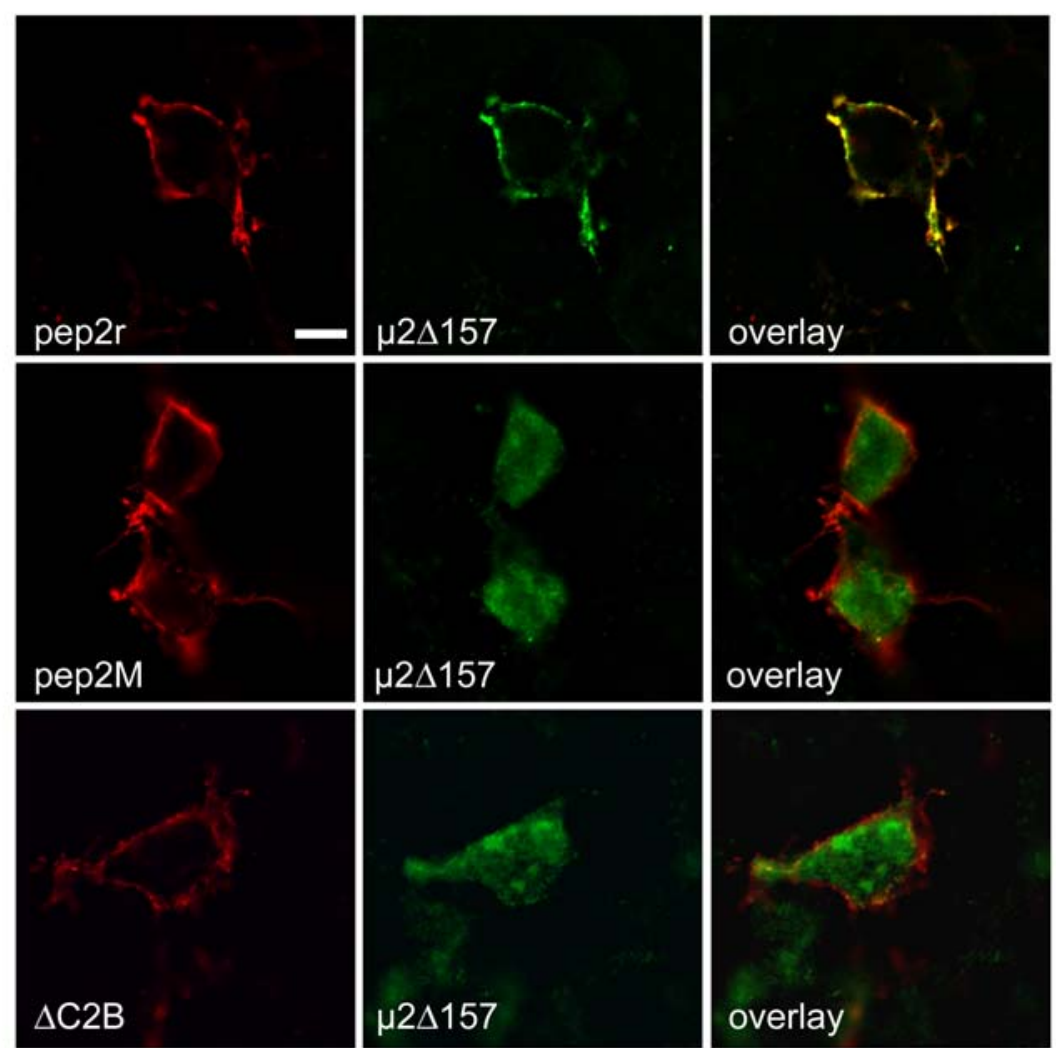

Figure 4.18: A synaptotagmin 1 chimera carrying the AMPA receptor basic sorting motif pep2r recruits soluble $\mu 2 \Delta 157$ to the plasma membrane of transfected HEK 293 cells. HEK cells were transfected alone (upper row) with a synaptotagmin 1 chimera carrying the AMPA receptor basic sorting motif FLAG-Syt1 $\triangle$ C2Bpep2r (pep2r), a mutant motif in which a lysine and an arginine where exchanged for alanines FLAG-Syt1 $\triangle$ C2Bpep2M (pep2M) and a truncated synaptotagmin 1 FLAG-Syt1 $\triangle \mathrm{C} 2 \mathrm{~B}(\triangle \mathrm{C} 2 \mathrm{~B})$ that were targeted to the plasma membrane and soluble HA- $\mu 2 \Delta 157(-\mu 2 \Delta 157)$ which was distributed in the cytoplasm. When cells were cotransfected with FLAG-Syt1 $\Delta$ C2Bpep2r (pep2r) in red and HA- $\mu 2 \Delta 157(\mu 2 \Delta 157)$ in green (upper panel) soluble $\mu 2$ is recruited to the plasma membrane by the synaptotagmin 1 chimera carrying the AMPA receptor basic sorting motif. A mutant chimera lacking the dibasic motif FLAGSyt1 $\Delta$ C2Bpep2M (pep2M) in red is unable to recruit $H A-\mu 2 \Delta 157(\mu 2 \Delta 157)$ in green to the plasma membrane of transfected cells (middle panel). Truncated synaptotagmin 1 devoid of the C2B domain Syt1 $\triangle$ C2B $(\Delta C 2 B)$ in red also does not affect the cytoplasmic distribution of HA$\mu 2 \Delta 157(\mu 2 \Delta 157)$ in green (lower panel). Scalebar: $5 \mu \mathrm{m}$ 
Plasma membrane recruitment of soluble $\mu 2$-adaptin by the synaptotagmin 1 chimeric construct FLAG-Syt1 $\Delta$ C2Bpep2r is critically dependent on two basic residues within the AMPA receptor basic sorting motif. Mutation of these residues to alanines in the mutant chimera FLAG-Syt1 $\Delta$ C2Bpep2M disrupts interaction with soluble $\mu 2$-adaptin in transfected cells.

These results are consistent with our data from previous in-vitro experiments and suggest that the direct interaction between the AMPA receptor and $\mu 2$ adaptin mediated by a dibasic recognition motif within the AMPA receptor cytoplasmic tail is responsible for membrane translocation of $\mu 2 \Delta 157$ seen in living cells. We have thus demonstrated that direct association of the AMPA receptor basic sorting motif pep2 $r$ and $\mu 2$-adaptin also functions in an in-vivo model. A putative implication for this interaction in clathrin-mediated endocytosis of the chimeric construct needs to be resolved in further experiments. 


\subsubsection{Internalization of a chimeric construct in transfected cells is mediated by the GluR2 basic sorting motif}

Based on the observation that soluble $\mu 2 \Delta 157$ is recruited to the plasma membrane of transfected cells by a synaptotagmin $1 \Delta \mathrm{C} 2 \mathrm{~B}$ chimeric construct carrying the AMPA receptor basic sorting motif pep2r. We next wanted to know if the dibasic sorting motif is sufficient to target the chimera for clathrin- and AP-2 dependent internalization.

Work of different groups has shown that AMPA receptor internalization can be induced by stimulation with agonists such as insulin, AMPA and NMDA. In primary hippocampal neurons endocytosis of the AMPA receptor subunit GluR1 upon insulin or AMPA stimulation occurs through a dynamin 1 dependent mechanism (Lin et al., 2000). There is evidence from work of Lee et al. (2002) that AMPA receptor internalization plays a role in NMDA induced longterm depression in primary hippocampal neurons.

GluR2 homomers from the plasma membrane of transfected HEK cells become internalized upon insulin stimulation (Man et al., 2000). Taken together these findings suggest that interaction between a basic motif in the GluR2 cytoplasmic tail and the AP-2 complex is needed for NMDA induced internalization of the AMPA receptor.

We show here that HA-tagged GluR2 homomers can also be internalized from the plasma membrane of transfected Cos7 cells. Cells were transfected with a GluR2 construct carrying an extracellular HA-tag. Plasma membrane receptor was live-labelled at $4{ }^{\circ} \mathrm{C}$ with primary $\alpha-\mathrm{HA}$ antibodies. Endocytosis of the receptor from the plasma membrane of transfected cells was induced by a brief stimulation with $0.5 \mu \mathrm{M}$ insulin. Cells were then allowed to undergo endocytosis of HA-GluR2 for additional 50 min. After fixation plasma membrane receptors were detected by staining with secondary Alexa594-conjugated antibodies. Cells were then permeabilized and internalized construct was labelled using Alexa488-conjugated secondary antibodies (fig. 4.19).

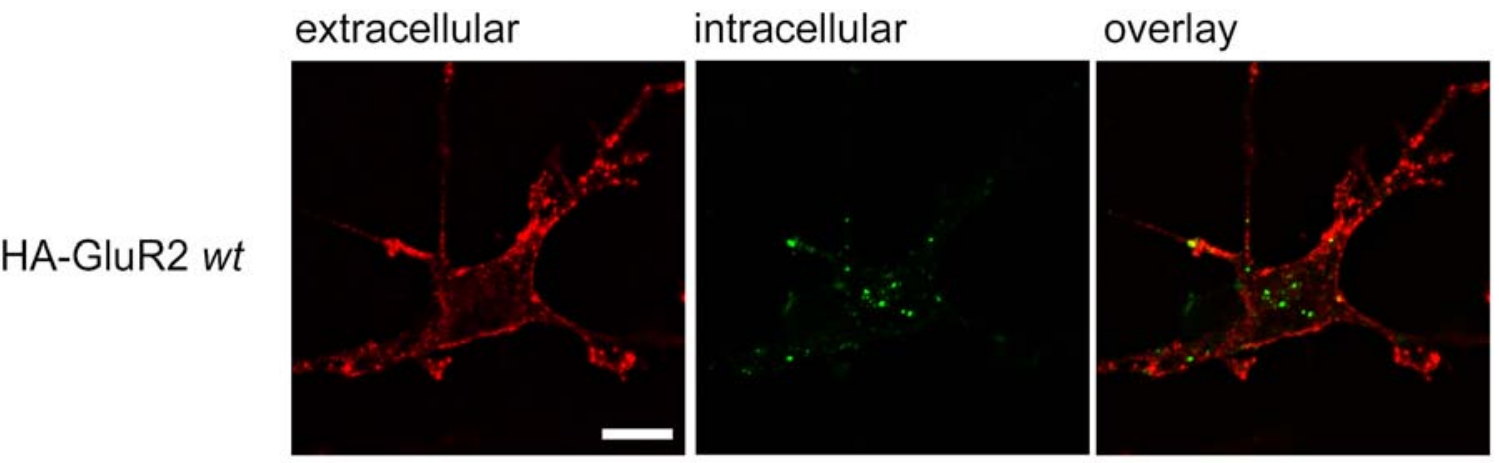

Fig. 4.19: Internalization of HA-GluR2 from the plasma membrane of transfected Cos7cells. The extracellular HA-tag of GluR2 wt transfected in Cos7 cells was labelled with monoclonal $\alpha-\mathrm{HA}$ antibody at $4{ }^{\circ} \mathrm{C}$. Internalization of the construct was stimulated by incubation with $0.5 \mu \mathrm{M}$ insulin for $10 \mathrm{~min}$ followed by an additional internalization period for $50 \mathrm{~min}$ at $37^{\circ} \mathrm{C}$. After fixation plasma membrane HA-GluR2 (red) was labelled under non-permeabilizing conditions with secondary Alexa594-conjugated goat-anti mouse. After blocking of the plasma membrane with unlabelled goat-anti mouse antibody internalized $\alpha-\mathrm{HA}$ antibody (green) was detected by labelling with Alexa488-conjugated goat-anti mouse under permeabilizing conditions. Scalebar: $15 \mu \mathrm{m}$ 
This system hence allowed us to compare the ability of native GluR2 receptor to become internalized with that of our chimeric fusion proteins and whether this required the AP-2 binding motif.

Therefore a chimeric construct was used in which the AMPA receptor basic sorting motif pep2r or a mutant motif comprising an alanine mutation of the dibasic cluster was fused to a truncated synaptotagmin 1 carrying an extracellular FLAG-tag. When expressed in a heterologeous cell culture system the chimera is sorted to the plasma membrane of transfected cells. Endocytosis of the construct was assayed by internalization of antibodies directed against the extracellular FLAG-tag as described before. Cos 7 cells expressing either the wildtype chimera (FLAG-Syt1 $\triangle$ C2Bpep2r), the mutant chimera (FLAGSyt1 $\Delta$ C2Bpep2M) or a truncated synaptotagmin 1 (FLAG-Syt1 $\Delta$ C2B) were live labelled with monoclonal $\alpha$-FLAG-antibodies at $4{ }^{\circ} \mathrm{C}$. Cells were then shifted to $37^{\circ} \mathrm{C}$ to allow internalization of the chimeric construct together with the antibody for $30 \mathrm{~min}$. After fixation cell surface labelling was detected by labelling with Alexa594- conjugated secondary antibodies (red fluorescence) under nonpermeabilizing conditions; unspecific labelling was blocked by application of non-labelled goat-anti mouse IgGs. Cells were TritonX-100 permeabilized and stained against internalized primary antibody using Alexa488-conjugated secondary antibodies (green fluorescence). Two transfections were carried out and two samples of each transfection were subjected to the antibody internalization assay resulting in four independent experiments. Internalization of the chimeric construct was quantified in about 20 transfected cells of each experiment by immunofluorescence microscopy and characterized as no, moderate or strong internalization (fig. 4.21). Representative cells for each construct FLAG-Syt1 $\Delta$ C2Bpep2r, FLAG-Syt1 $\Delta$ C2Bpep2M and FLAG-Syt1 $\Delta$ C2B are shown in fig. 4.20.

The wildtype chimeric construct FLAG-Syt1 $\Delta$ C2Bpep2r (upper panel) carrying the AMPA receptor dibasic sorting motif was endocytosed in the majority

$(69 \pm 9 \%)$ of the transfected cells. One third $(29 \pm 10 \%)$ of the transfected cells showed strong internalization whereas the construct was moderately endocytosed in $40 \pm 7 \%$ of the transfected cells. No endocytosis of FLAGSyt1 $\Delta$ C2Bpep2r did occur in $31 \pm 10 \%$ of the transfected cells.

Mutation of a dibasic motif into alanines in (FLAG-Syt1 $\Delta$ C2Bpep2M) (middle panel) resulted in a decrease of endocytosis from the plasma membrane: $62 \pm 5$ $\%$ of transfected cells did not internalize the construct. Moderate internalization, presumably by bulk endocytosis, was seen in $34 \%$ of the transfected cells. Strong endocytosis of the construct was completely abolished by the mutation and could be detected only in $4 \%$ of the transfected cells.

A truncated synaptotagmin 1 (FLAG-Syt1 $\Delta$ C2B) lacking its C2B domain (lower panel) which harbours a basic endocytic sorting motif showed only weak internalization from the plasma membrane of transfected cells. $76 \pm 8 \%$ of transfected cells did not endocytose, moderate internalization occurred in $16 \pm$ $4 \%$ and strong internalization in $5 \pm 4 \%$ of the transfected cells.

These results demonstrate that the GluR2 basic sorting motif is sufficient to target endocytosis of a chimeric construct from the plasma membrane of heterologeously transfected cells and that internalization of the construct is strongly dependent on a dibasic motif within the peptide that is recognized by the $\mu 2$-subunit of the AP-2 adaptor complex in-vitro and in-vivo. 


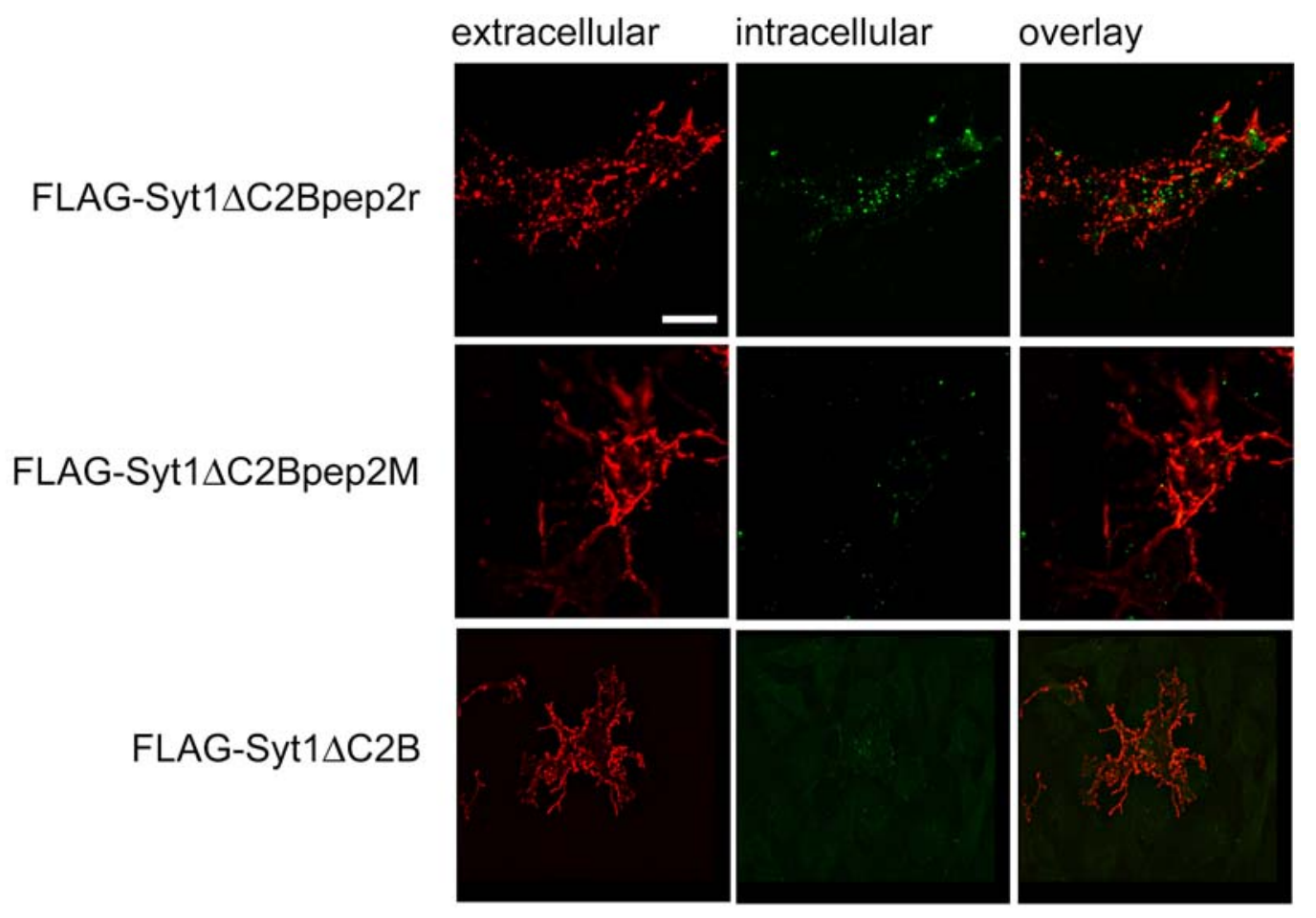

Fig. 4.20: The AMPA receptor basic sorting motif targets internalization of a chimeric construct from the plasma membrane of transfected cells. Cos 7 cells were transfected with synaptotagmin 1 chimeric construct in which the C2B-domain was replaced by the AMPA receptor basic sorting motif (FLAG-Syt1 $\triangle$ C2Bpep2r), a mutant motif carrying two alanines instead of the dibasic cluster (FLAG-Syt1 $\triangle$ C2Bpep2M), and a truncated synaptotagmin (FLAGSyt1 $\Delta$ C2B). Cos 7 expressing the chimeric construct were live labelled with a monoclonal antibody directed against the extracellular FLAG-tag for $45 \mathrm{~min}$ at $4{ }^{\circ} \mathrm{C}$. The temperature was then shifted to $37^{\circ} \mathrm{C}$ to allow endocytosis of the construct together with the primary antibody for $30 \mathrm{~min}$. After fixation extracellular $\alpha$-FLAG primary antibody at the plasma membrane of transfected cells was labelled with Alexa594 conjugated secondary antibody (red) under nonpermeabilizing conditions. Endocytosed $\alpha$-FLAG primary antibody was detected after TritonX100 permeabilization of the cells by application of Alexa488-conjugated secondary antibody (green). Scalebar: $15 \mu \mathrm{m}$

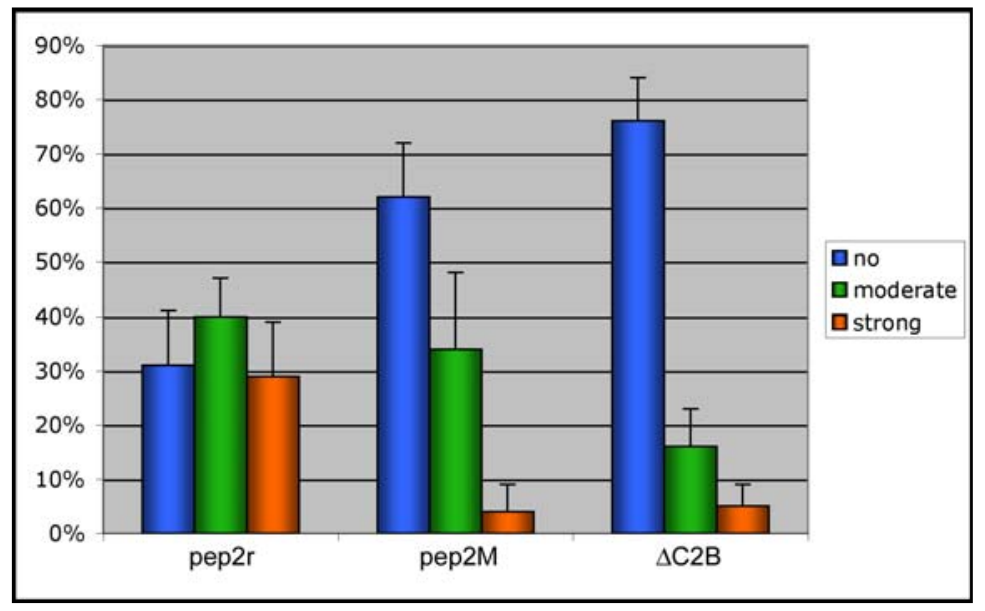

Fig. 4.21: Quantitative analysis of FLAG-Syt1 $\triangle$ C2Bpep2r, FLAG-Syt1 $\triangle$ C2Bpep2M and FLAG-Syt1 $\triangle C 2 B$ endocytosis in transfected Cos7 cells. Internalization of the constructs in transfected $\operatorname{Cos} 7$ cells was assayed by immunofluorescence microscopy and classified into no, moderate or strong internalization. The histogram shows the averaged percentage of transfected cells over 4 independent experiments $(\mathrm{N}=4)$ for each construct which undergo no, moderate or strong internalization. Error bars give the mean standard deviation. 


\subsubsection{Depletion of $\mu 2$-adaptin results in a decreased internalization of the synaptotagmin 1 chimeric construct carrying the AMPA receptor basic sorting motif pep2r}

In previous experiments we have demonstrated direct interaction between the synaptotagmin 1 chimera carrying the AMPA receptor basic sorting motif and $\mu 2$-adaptin. critically Internalization of the chimera expressed in a heterologeous context is strongly dependent on the dibasic cluster within the AP-2 recognition motif in GluR2. By inhibition of clathrin-mediated endocytosis in cells transfected with siRNAs against $\mu 2$-adaptin we aimed to interfere with the internalization of synaptotagmin 1 chimera carrying the AMPA receptor basic sorting motif.

SiRNA directed against $\mu 2$-adaptin provides an effective tool to knock-down clathrin-mediated endocytosis in transfected cells (Motley et al., 2003; Hinrichsen et al., 2003). Depletion of $\mu 2$-adaptin in the transfected cells resulted in a decreased number of clathrin-coated pits and blocked internalization of external added transferrin by clathrin mediated endocytosis (Motley et al., 2003; Hinrichsen et al., 2003).

Cos7 cells were co-transfected with siRNA targeted against $\mu 2$-adaptin or a control siRNA and FLAG-Syt1 $\triangle$ C2Bpep2r. The successfull knock-down of $\mu 2-$ adaptin in Cos7 cell extracts was assayed by SDS-PAGE and immunoblotting against $\mu 2$-adaptin (fig. 4.22).

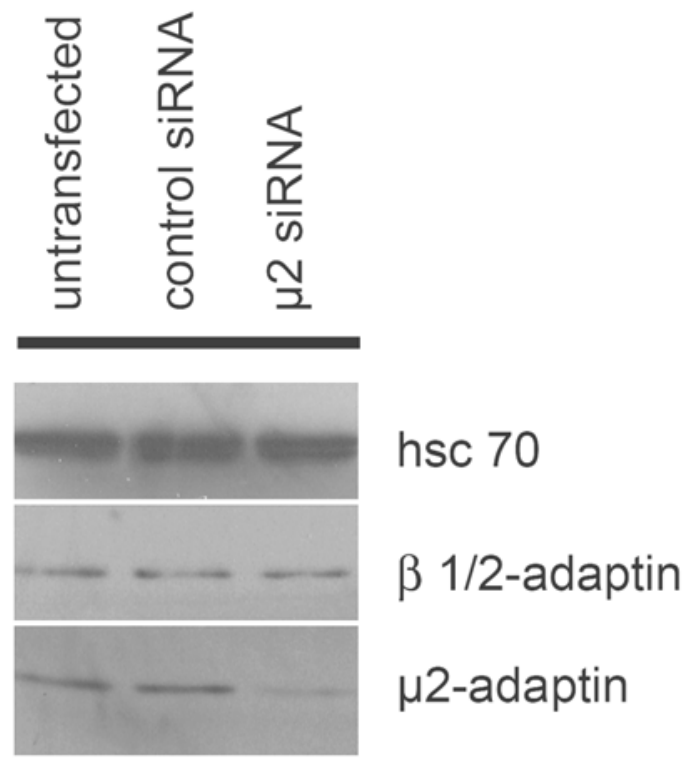

Fig. 4.22: Specific depletion of $\mu 2$-adaptin in Cos 7 cells. Cos 7 cells were transfected once with small interferring RNA oligonucleotides directed against $\mu 2$-adaptin or a control siRNA. Specific depletion of $\mu 2$-adaptin was analyzed in $10 \mu \mathrm{g}$ of Triton-X 100 extracted cell homogenates by SDS-PAGE and immunoblotting against heat shock chaperon 70 (hsc 70), $\beta$ 1/2-adaptin and $\mu 2$-adaptin.

SiRNA directed against $\mu 2$-adaptin specifically blocks its expression whereas expression levels of hsc 70 or $\beta$ 1/2-adaptin were not decreased in the transfected cells. Transfection of a control siRNA did not affect the expression levels of hsc $70, \beta 1 / 2$ - or $\mu 2$-adaptin in the transfected cells.

Thus, siRNA mediated knock-down of $\mu 2$-adaptin provided a valuable tool to address the question if internalization of the synaptotagmin 1 chimera from the plasma membrane of transfected cells was dependent on the recognition of the AMPA receptor basic sorting motif by $\mu 2$-adaptin (fig. 4.23): 


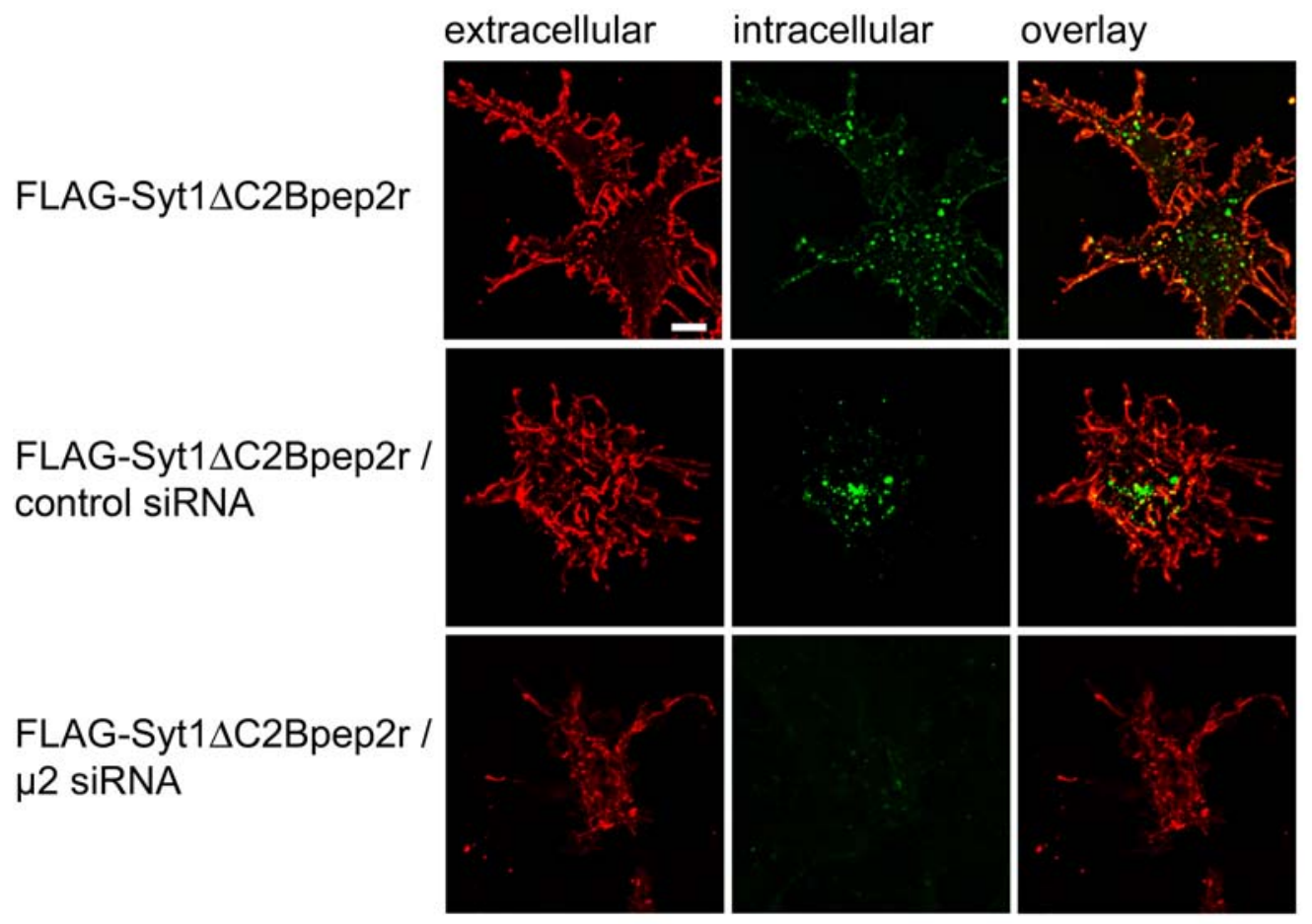

Fig. 4.23: Depletion of $\mu 2$-adaptin impaires clathrin-mediated endocytosis of a synaptotagmin 1 chimera carrying the AMPA receptor basic sorting motif. Cos 7 cells were transfected either with synaptotagmin 1 chimeric construct in which the C2B-domain was replaced by the AMPA receptor basic sorting motif (FLAG-Syt1 $\triangle$ C2Bpep2r) alone or together with a control siRNA or siRNA directed against $\mu 2$-adaptin. Cos 7 expressing the chimeric construct alone or together with the siRNAswere live labelled with a monoclonal antibody directed against the extracellular FLAG-tag for $45 \mathrm{~min}$ at $4{ }^{\circ} \mathrm{C}$. The temperature was then shifted to $37{ }^{\circ} \mathrm{C}$ to allow endocytosis of the construct together with the primary antibody for $30 \mathrm{~min}$. After fixation extracellular $\alpha$-FLAG primary antibody at the plasma membrane of transfected cells was labelled with Alexa594 conjugated secondary antibody (red) under non-permeabilizing conditions. Endocytosed $\alpha$-FLAG primary antibody was detected after TritonX-100 permeabilization of the cells by application of Alexa488-conjugated secondary antibody (green). Scalebar: $15 \mu \mathrm{m}$

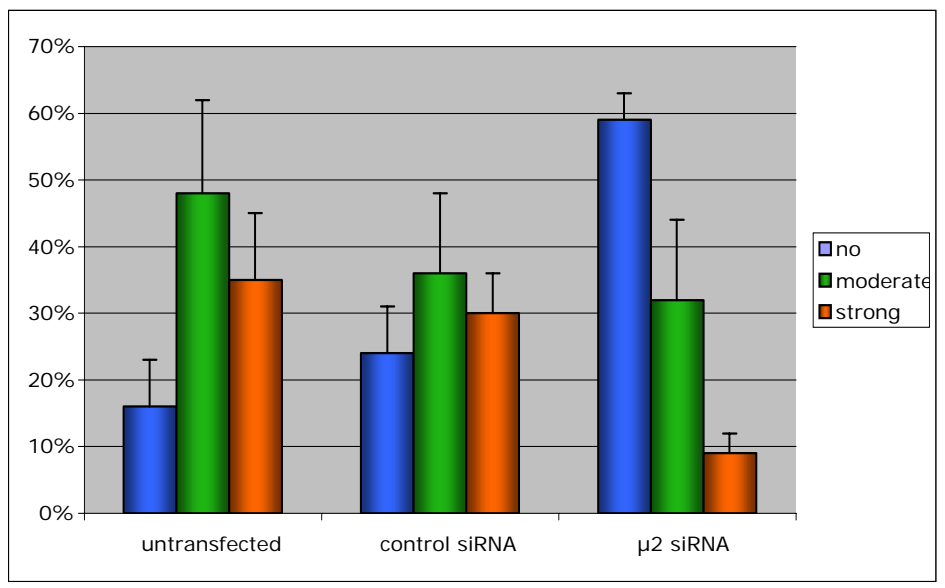

Fig 4.24: Quantitative analysis of FLAG-Syt1 $\triangle$ C2Bpep2r internalization in Cos7 cells depleted of $\boldsymbol{\mu 2}$-adaptin. Internalization of the construct in transfected Cos7 cells was assayed by immunofluorescence microscopy and classified into no, moderate or strong internalization. The histogram shows the averaged percentage of transfected cells over 4 independent experiments $(\mathrm{N}=4)$ for each construct which undergo no, moderate or strong internalization. Error bars give the mean standard deviation. 
Internalization of the FLAG-tagged synaptotagmin 1 chimera in transfected cells was assayed by an antibody internalization assay as described before

(fig. 4.24). Plasma membrane expressed FLAG-Syt1 $\Delta$ C2Bpep2r was labelled by Alexa594-conjugated secondary antibodies (red fluorescence) under nonpermeabilizing conditions. Internalized construct was labelled using Alexa488conjugated secondary antibodies (green fluorescence).

FLAG-Syt1 $\triangle$ C2Bpep2r (upper panel) alone or co-transfected with control siRNA (middle panel) was effectively internalized from the plasma membrane of transfected cells. siRNA mediated depletion of $\mu 2$-adaptin decreased the internalization of FLAG-Syt1 $\triangle$ C2Bpep2r from the plasma membrane of transfected cells (lower panel).

Endocytosis of the construct from the plasma membrane of transfected cells was assayed by immunocytochemistry. Levels of internalization were classified into no, moderate or strong (fig. 4.24). For each condition internalization in 20 to 25 transfected cells from 4 independent experiments was assayed.

In control cells the chimeric construct was effictively internalized from the plasma membrane of transfected cells: $35 \pm 10 \%$ showed strong and $48 \pm 14 \%$ moderate internalization of the construct from the plasma membrane. Only $16 \pm$ $7 \%$ of the transfected cells did not endocytose the construct.

Co-transfection with a control siRNA did not affect clathrin-mediated endocytosis of the chimeric construct. Strong internalization was seen in $30 \pm 6 \%$ and moderate endocytosis in $36 \pm 12 \%$ of the transfected cells.

$24 \pm 7 \%$ of the transfected cells did not undergo internalization of the synaptotagmin 1 chimera.

Depletion of $\mu 2$-adaptin specifically inhibits clathrin-mediated endocytosis of the synaptotagmin 1 chimera by inhibitng the recognition of the basic AMPA receptor sorting motif by the clathrin-adaptor AP-2. The majority (59 $\pm 4 \%$ ) of $\mu 2$-depleted cells did not endocytose the synaptotagmin 1 chimera from their plasma membrane. Subsequently the level of strong internalization of the synaptotagmin 1 chimera from the plasma membrane of $\mu 2$-depleted cells was decreased to $9 \pm 3 \%$ in comparison to control cells. A constant level of moderate internalization was seen in $32 \pm 12 \%$ of the transfected cells.

siRNA directed against $\mu 2$-adaptin provides a specific tool to deplete CME in cultured cells (Motley et al., 2003; Hinrichsen et al., 2003). This experiment clearly shows that the AMPA receptor dibasic sorting motif targets clathrinmediated endocytosis by directly binding to the $\mu 2$-subunit of the clathrinadaptor AP-2. Thus internalization of native AMPARs is both dependent on the dibasic recognition motif within the GluR2 cytoplasmic tail and intact AP-2 heterotetramers.

These findings imply that recognition of the dibasic sorting motif within the GluR2 cytoplasmic tail by $\mu 2$-adaptin mediates clathrin-dependent internalization of the AMPAR in agonist induced longterm depression in the central nervous system. 


\subsection{Sorting of SV proteins during neuronal differentiation}

\subsubsection{Expression of genes encoding for synaptic vesicle proteins is switched on at different time points during neuronal differentiation of P19 cells}

Neuronal differentiation requires the establishment of axonal and somatodendritic polarity in an unpolarized precursor cell. This process includes formation of a protein sorting machinery that mediates correct sorting of proteins to their axonal or dendritic target.

In neurondocrine cells the synaptic vesicle protein synaptophysin was found to co-localize with the transferrin receptor in an endocytic recycling compartment (Cameron et al., 1991). As mentioned before clathrin-mediated endocytosis plays an important role in the recycling of synaptic vesicle proteins from the presynaptic plasma membrane after neurotransmitter release (Heuser \& Reese, 1973). Out of these observations the question arises if clathrin-mediated recycling of synaptic vesicle proteins plays a role in the establishment of axonal polarity during neuronal differentiation.

Studies on primary hippocampal neurons revealed that expression of synaptic vesicle proteins occurs prior to neurite outgrowth and synapse formation (Fletcher et al., 1994; Marazzi \& Buckley, 1993). Expression of synaptic vesicle proteins in primary hippocampal neurons from very early stages impaires studies on de-novo synaptic vesicle biogenesis. Therefore retinoic acid induced neuronal differentiation of pluripotent embryonic carcinoma cells was used as a tool to study formation of synaptic vesicles.

First, we were interested in the temporal pattern of gene expression encoding for synaptic vesicle proteins during neuronal differentiation of P19 cells. Therefore we isolated RNA from P19 precursor cells, cell aggregates $24 \mathrm{~h}$ and 4 days after retinoic acid induction and from mature P19 neurons. RNA was reverse transcribed into cDNA and amplified by PCR using specific primers specific for SV protein encoding cDNA or ubiquitously expressed $\beta$-actin (fig. 4.25).

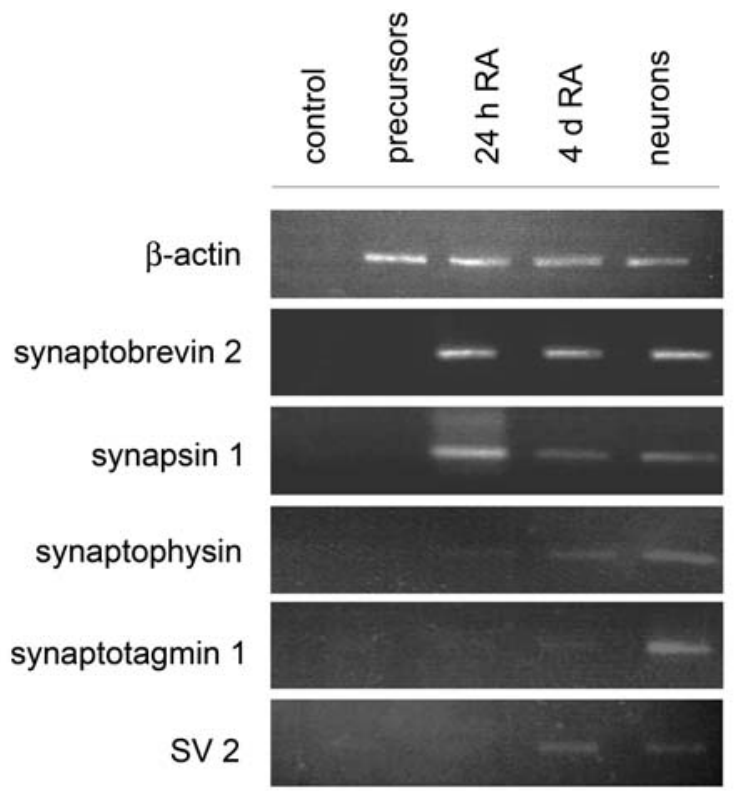

Fig. 4.25: Expression of genes encoding for synaptic vesicle proteins is switched on at different time points during neuronal differentiation of P19 cells. RNA was isolated from P19 precursors, cell aggregates $24 \mathrm{~h}$ and 4 days after retinoic acid induction and $P 19$ neurons an reverse transcribed. cDNA amounts were estimated by PCR using primers against ubiquitiously expressed $\beta$-actin. Expression of genes encoding for synaptic vesicle proteins was analyzed by PCR using primer pairs specific for synaptobrevin $\quad 2, \quad$ synapsin, synaptophysin, synaptotagmin 1 and SV2. 
Expression of the SNARE protein synaptobrevin 2 and the synaptic vesicle protein synapsin 1 could be detected shortly after retinoic acid induction of neuronal differentiation. Gene expression of synaptobrevin 2 and synapsin did not increase during the differentiation process. In contrast expression of genes encoding for synaptophysin, synaptotagmin 1 and SV2 was initiated at later stages and the expression level was up-regulated during neuronal differentiation.

These findings show that there is a differential expression pattern for mRNAs of synaptic vesicle proteins during neuronal differentiation of P19 cells and that expression of some genes i. e. synaptobrevin 2 and synapsin already starts at an early time point of differentiation whereas genes encoding for other synaptic vesicle proteins such as synaptotagmin 1 are expressed at a later stage of neuronal differentiation.

\begin{tabular}{|c|c|c|c|c|}
\hline & precursors & $\begin{array}{l}24 \mathrm{~h} \text { RA cell } \\
\text { aggregates }\end{array}$ & $\begin{array}{l}\text { d d RA cell } \\
\text { aggregates }\end{array}$ & neurons \\
\hline$\beta$-actin & + & + & + & + \\
\hline $\begin{array}{l}\text { synaptobrevin / } \\
\text { VAMP } 2\end{array}$ & - & + & + & + \\
\hline $\begin{array}{l}\text { TI-VAMP / } \\
\text { VAMP } 7\end{array}$ & + & + & + & + \\
\hline synapsin 1 & - & + & + & + \\
\hline synaptophysin & - & $-I+$ & + & ++ \\
\hline synaptotagmin 1 & - & - & $-1+$ & + \\
\hline SV 2 & - & - & + & + \\
\hline dynamin 1 & - & $-1+$ & + & + \\
\hline $\begin{array}{l}\text { AP-2 } \\
\mu 2 \text {-adaptin }\end{array}$ & + & + & + & + \\
\hline AP3 $\sigma 3$-adaptin & + & + & + & + \\
\hline stonin 2 & - & + & + & + \\
\hline arf 6 & + & + & + & + \\
\hline $\begin{array}{l}\text { HMG-CoA- } \\
\text { reductase }\end{array}$ & $-I+$ & + & + & + \\
\hline PIP-kinase $1 \gamma$ & - & - & - & + \\
\hline $\begin{array}{l}\text { plasma- } \\
\text { membrane- } \\
\text { ganglioside- } \\
\text { sialidase }\end{array}$ & - & $-1+$ & + & + \\
\hline
\end{tabular}

Table 4.1: Gene expression pattern of synaptic vesicle proteins, endocytic proteins and cholesterol and sphingomyelin generating enzymes during neuronal differentiation of P19 cells

The gene expression pattern of several proteins belonging to the endocytosis machinery was also analyzed by RT-PCR (table 5B): The clathrin-adaptor AP-2 was expressed at a constant level whereas gene expression of the GTPase dynamin 1, the accessory protein stonin 2, the small GTPase arf6 and the PI4,5-bisphosphate generating enzyme PIP-kinase $1 \gamma$ were up-regulated during neuronal differentiation of P19 cells.

Since lipid raft formation seems to be involved in polarization of neuronal precursor cells (Ledesma et al. 1999, Thiel et al. 2000) the gene expression patterns of HMG-CoA reductase an enzyme catalyzing the rate limiting step of cholesterol formation and plasma-membrane-ganglioside-sialidase which is involved in sphingomyelin biosynthesis were also characterized (Table 1B). 
Gene expression of HMG-CoA reductase was slightly up-regulated during neuronal differentiation. mRNA encoding for plasma-membrane-gangliosidesialidase was detected at a later stage of neuronal differention and the expression level was increased in $4 \mathrm{~d}$ old aggregates and P19 neurons.

The next experiments addressed the question if expression of synaptic vesicle proteins during neuronal differentiation followed the same temporal order as the expression of the corresponding genes. Therefore the intracellular distribution of synaptic vesicle proteins in dissociated cell aggregates fixed $24 \mathrm{~h}$ or $4 \mathrm{~d}$ after retinoic acid induction and in mature P19 neurons was analyzed by immunocytochemistry (fig. 5.26).

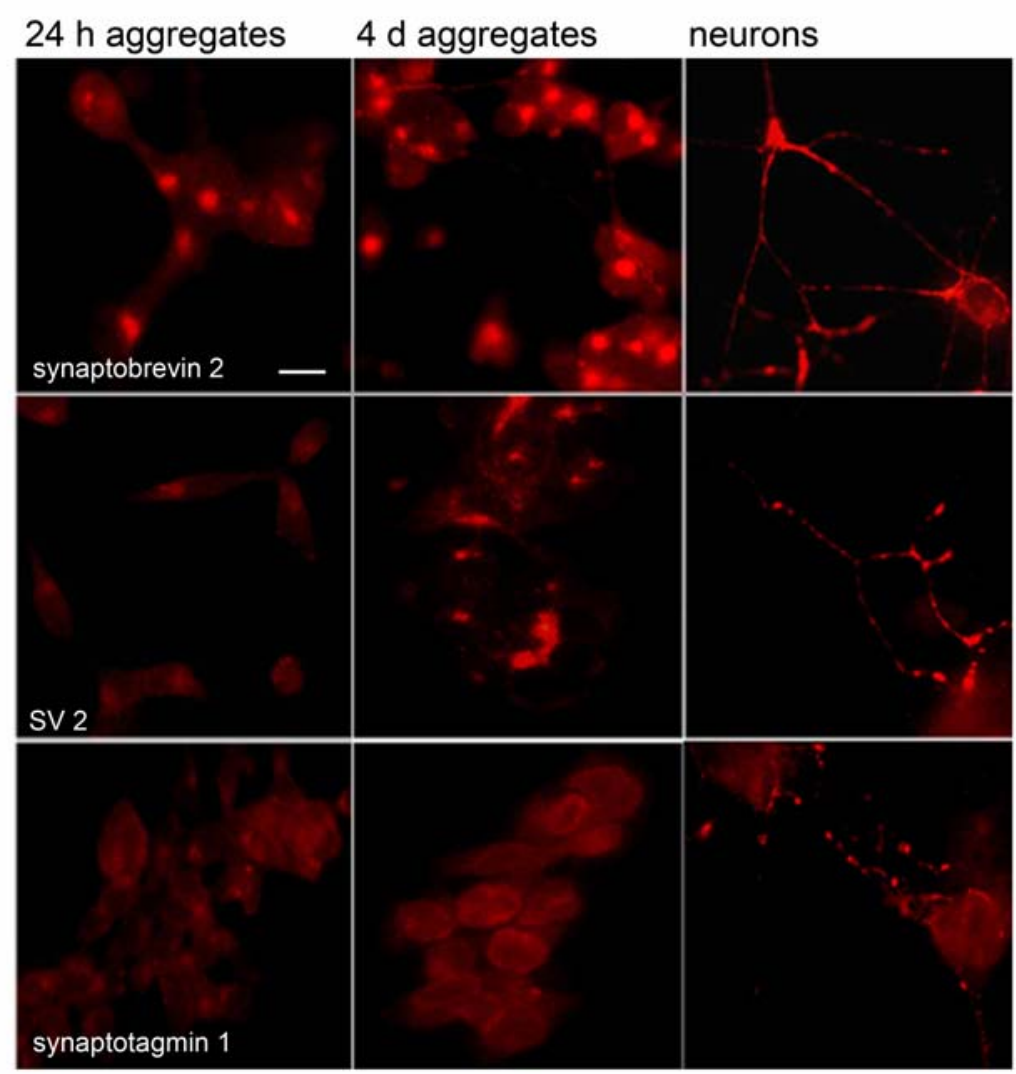

Fig. 4.26: Synaptic vesicle proteins synaptobrevin 2, SV 2 and synaptotagmin 1 are expressed at different time points during neuronal differentiation of P19 cells. Dissociated P19 cells aggregates $24 \mathrm{~h}$ and $4 \mathrm{~d}$ after retinoic acid induction and P19 neurons were seeded for immunocytochemistry on Matrigel coated coverslips. Cells were fixed with PFA and expression of synaptic vesicle proteins was analyzed by staining for synaptobrevin 2, SV 2 and synaptotagmin 1. Scalebar: $20 \mu \mathrm{m}$

Expression of synaptobrevin 2 was already detected $24 \mathrm{~h}$ after retinoic acid induction. The protein localized to a perinuclear compartment in P19 neuronal precursors; in mature P19 neurons it showed a punctate distribution in processes. According to the gene expression pattern SV 2 was found in neuronal precursor cells 4 days after retinoic acid induction where it was located to a perinuclear compartment similar to synaptobrevin 2. In P19 neurons SV2 also localized to puncta in the axon which are presumably synaptic boutons. 
The expression level of synaptotagmin $14 \mathrm{~d}$ after retinoic acid induction was low compared to synaptobrevin which was expressed in most neuronal precursor cell.

Taken together the expression pattern of synaptic vesicle proteins during neuronal differentiation of P19 cells characterized by immunocytochemistry confirmed the gene expression pattern found by RT-PCR: Synaptobrevin 2 expression was switched on already $24 \mathrm{~h}$ after retinoic acid induction and was present in nearly all neuronal precursor cells where the protein localized to a perinuclear compartment. In contrast, expression of SV 2 and synaptotagmin 1 on mRNA and protein level was detected at later differentiation stages. All synaptic vesicle proteins localized to a perinuclear compartment in the neuronal precursor cells and showed a punctate distribution in the axons of mature P19 neurons. 


\subsubsection{Synaptic vesicle proteins co-localize to the axon and a perinuclear compartment in P19 neurons}

In P19 neuronal precursors synaptic vesicle proteins localized to a perinuclear compartment whereas their distribution showed a punctate pattern in the processes of mature P19 neurons. By immunocytochemistry co-localization of the synaptic vesicle markers synaptobrevin 2, SV2, synaptotagmin 1 and synaptophysin in mature P19 neurons was characterized (Fig. 4.27).
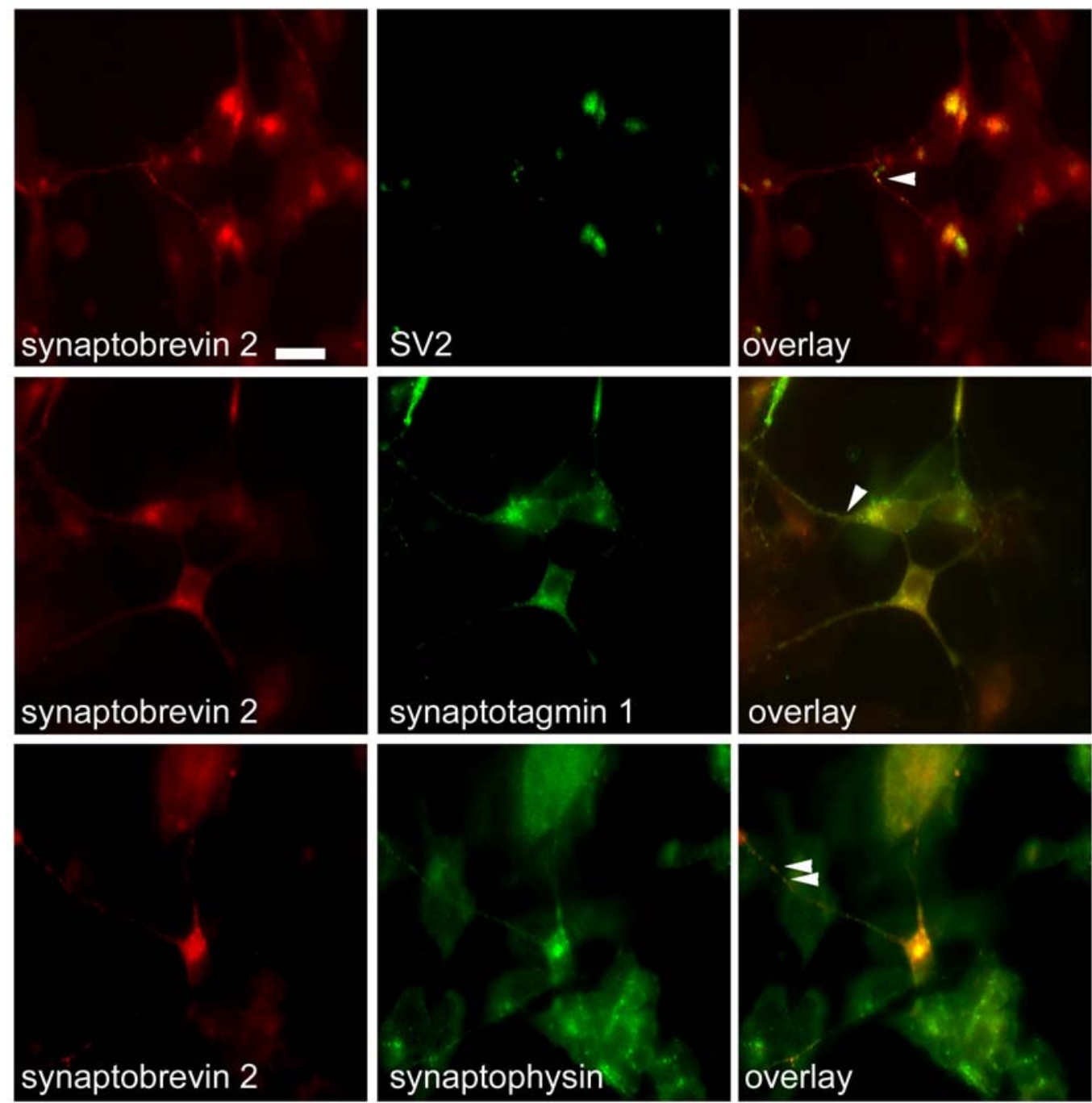

Fig. 4.27: Synaptic vesicle proteins synaptobrevin 2, SV2, synaptotagmin 1 and synaptophysin co-localize in a perinuclear compartment and the axons of P19 neurons. P19 neurons were seeded for immunocytochemistry on Matrigel coated coverslips. Cells were fixed with PFA and intracellular distribution of synaptic vesicle proteins was analyzed by colabelling of synaptobrevin 2 (red) with SV 2 green (upper panel). Synaptobrevin 2 (red) also colocalizes with synaptotagmin 1 (green, middle panel) and synaptophysin (green, lower panel). Arrowheads indicate co-localization with synaptobrevin 2 in the axon of a P19 neuron. Scalebar: $20 \mu \mathrm{m}$

The SNARE protein synaptobrevin 2 (red) was abundantly expressed in P19 neuronal precursors (see section 1) and mature P19 neurons. It co-localized with the synaptic vesicle marker SV2 (green, upper panel) to a perinuclear 
compartment and punctate structures which represent synaptic boutons to the axon of mature P19 neurons.

Synaptobrevin 2 (red) and synaptotagmin 1 (green) also showed co-localization to the perinuclear compartment and in the axon of P19 neurons (middle panel).

The synaptic vesicle marker synaptophysin (green, lower panel) located to the perinuclear compartment and to synaptic boutons in the axon of P19 neurons similar to synaptobrevin 2 (red), synaptotagmin 1 and SV2.

Since different synaptic vesicle proteins showed the same intracellular distribution to a perinuclear compartment and synaptic boutons in the axons of mature P19 neurons synaptobrevin 2 was chosen as a representative marker for synaptic vesicles and the perinuclear compartment in the following experiments. 


\subsubsection{A perinuclear compartment containing synaptic vesicle proteins in P19 neuronal precursors does not originate from the Golgi-apparatus}

In P19 neuronal precursor cells 7 days after retinoic acid induction which do not have any processes yet the synaptic vesicle marker synaptobrevin 2 was found to localize to a perinuclear compartment. We were interested if this compartment was a part of the Golgi apperatus and therefore analyzed colocalization of synaptobrevin 2 with the Golgi matrix protein GM130 in 7 days old P19 neuronal precursor cells by immunocytochemistry. The perinuclear compartment to which synaptobrevin 2 (red fluorescence) localizes seems to be surrounded by the Golgi and showed a partially overlapping distribution with the Golgi marker GM130 (green fluorescence) in the neuronal precursor cells (fig. 4.28 upper panel).

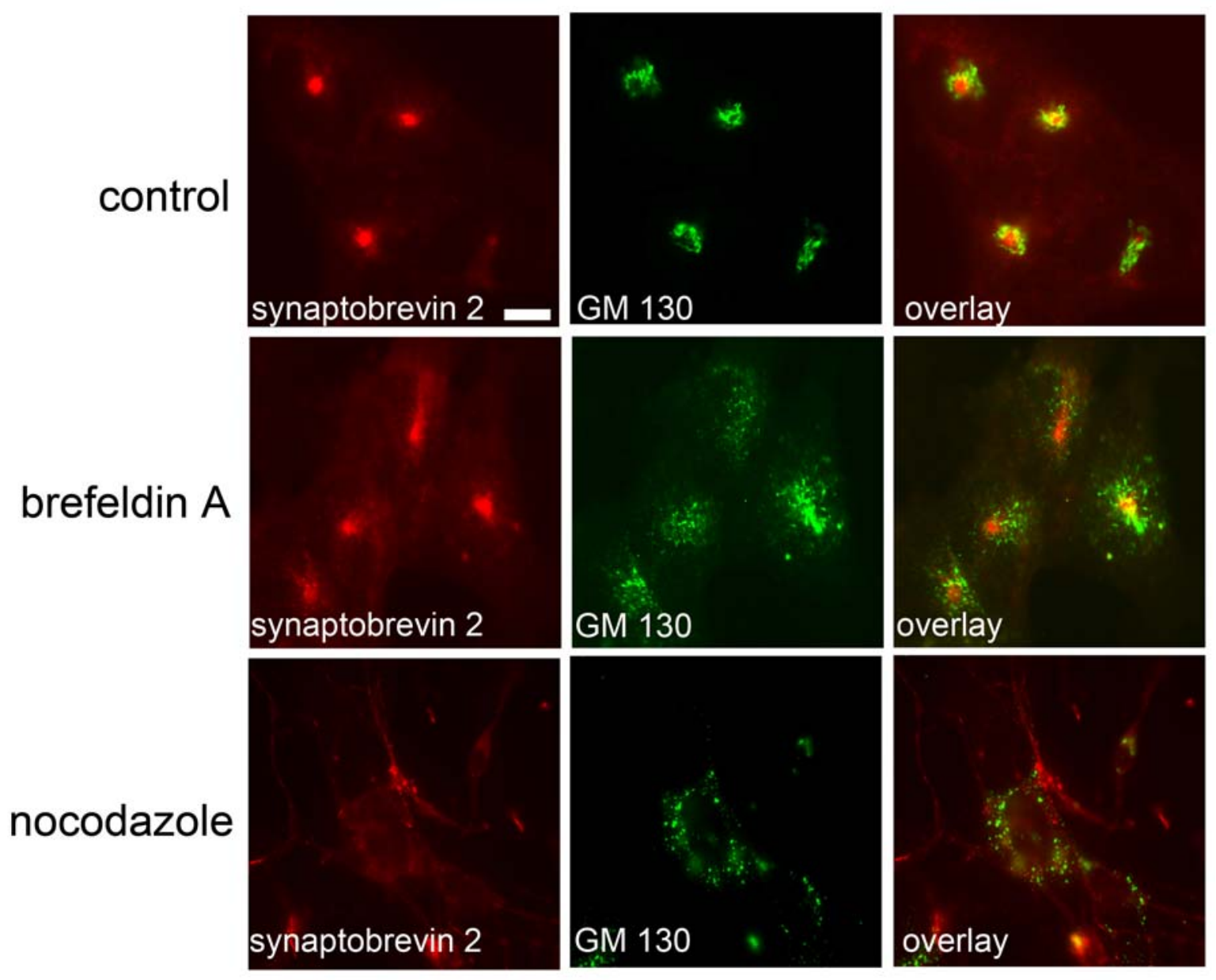

Fig. 4.28: Disruption of the Golgi apperatus does not affect the intracellular distribution of synaptobrevin 2 in P19 neuronal precursor cells. The upper panel shows co-labelling of synaptobrevin 2 (red) and the Golgi marker GM 130 (green) in d7 P19 neuronal precursor cells. To disrupt the Golgi P19 neuronal precursors were treated for 60 min at $37{ }^{\circ} \mathrm{C}$ with $\mu \mathrm{g} / \mathrm{ml}$ brefeldin A (middle panel). Cells were fixed with PFA and stained against synaptobrevin 2 (red) and GM 130 (green). Microtubule and the Golgi were disrupted by incubating the cells for 60 min at $37{ }^{\circ} \mathrm{C}$ with $500 \mathrm{nM}$ nocodazole (lower panel). Cells were fixed with PFA and stained against synaptobrevin 2 (red) and GM 130 (green). Scale bar: $20 \mu \mathrm{m}$ 
Because we could not exclude a possible Golgi origin of the perinuclear compartment by co-immunolabelling against synaptobrevin 2 the question if disruption of the Golgi would also affect the intracellular distribution of synaptobrevin 2 in P19 neuronal precursors was addressed. Therefore cells were treated with the fungal metabolite brefeldin A (BFA) which blocks the assembly of coatamer by inhibiting the GTP-dependent interaction of ARF with the Golgi membrane (Donaldson et al., 1992). BFA treatment lead to defragmentation of the Golgi and a scattered staining against GM130 throughout the cell (fig. 4.28 middle panel, green fluorescence).

Intracellular distribution of synaptobrevin 2 (middle panel, red) to the perinuclear compartment was not affected by BFA.

Treatment of P19 neuronal precursors with nocodazole a microtubule depolymerizing agent also resulted in defragmentation of the Golgi apperatus and a scattered GM130 staining (fig. 4.28 lower panel, green fluorescence). The punctate labelling of synaptobrevin 2 (lower panel, red fluorescence) in the processes of P19 neuronal precursors and its localization to the perinuclear compartment was not disrupted by treating the cells with nocodazole.

These observations implicate that the perinuclear compartment containing synaptobrevin 2 in P19 precursor cells does not originate from the Golgi apperatus although its intracellular distribution is overlapping with the Golgi matrix protein GM 130. So the organellar nature of this compartment needs to be addressed in further co-localization experiments with other organellar markers. 


\subsubsection{Analysis of a putative endosomal recycling compartment containing synaptic vesicle proteins in neuronal precursor cells}

Since the synaptic vesicle protein containing perinuclear compartment in P19 neuronal precursor cells was not of Golgi origin we were interested if it was a compartement derived of the endocyitic pathway. Studies at neuroendocrine cells hint at an implication of the endocytic pathway for the formation of synaptic vesicle like microvesicles (SLMVs): A study of Cameron et al. (1991) describes two different vesicle populations which contain synaptophysin in PC12 cells. In one of these vesicle pools synaptophysin was found to co-localize with the transferrin receptor a characteristic marker for endosomes. Work from Povoda et al. (1999) on PC12 confirms these findings: They characterized a vesicular compartment in which synaptic vesicle proteins co-localize with different transporter proteins and the transferrin receptor. Both studies propose that sorting endosomes function as an intermediate compartment in synaptic vesicle biogenesis.

A well established tool to study clathrin-mediated endocytosis in cells is internalization of fluorescently labelled transferrin. This approach was used to address the question if transferrin would be targeted to the synaptic vesicle protein containing perinuclear compartment in P19 neuronal precursors (Fig. 4.29).
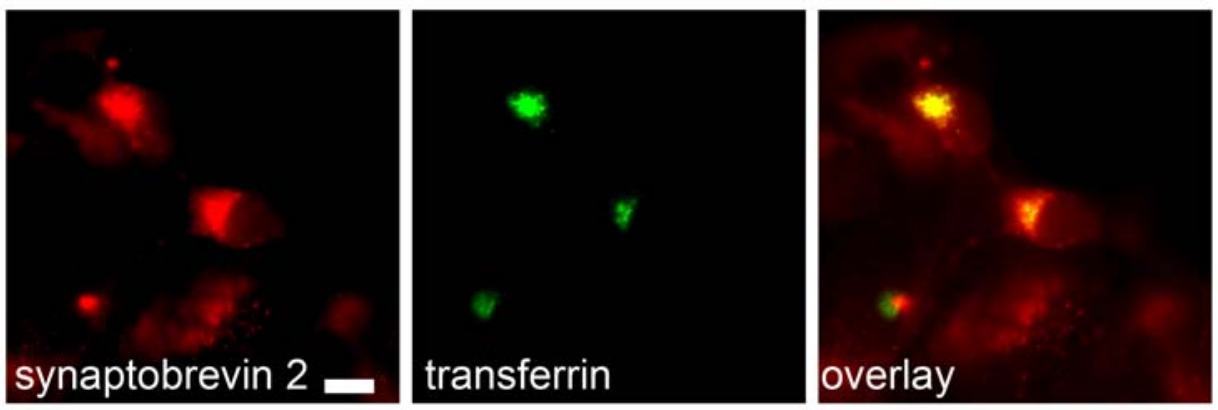

Fig. 4.29: Internalized transferrin is targeted to the synaptic vesicle protein containing perinuclear compartment in P19 neuronal precursors. P19 neuronal precursors were incubated for $30 \mathrm{~min}$ at $30{ }^{\circ} \mathrm{C}$ with $10 \mu \mathrm{g} / \mathrm{ml}$ Alexa488-conjugated transferrin (green). After an acidic wash to remove cell surface labelling cells were fixed and stained against synaptobrevin 2 (red). Scalebar: $20 \mu \mathrm{m}$

P19 cells were allowed to internalize Alexa488 conjugated transferrin for $30 \mathrm{~min}$ at

$37{ }^{\circ} \mathrm{C}$. Fixed cells were stained for synaptobrevin 2 . We found that after $30 \mathrm{~min}$ of internalization transferrin (green) was transported to the perinuclear compartment where it co-localized with synaptobrevin 2.

This finding indicates an endosomal origin of the compartment therefore we analyzed co-localization of the perinuclear compartment with markers for early endosomes and a late recycling endosome by immunocytochemistry of P19 neuronal precursors.

Labelling against the early endosomal antigen EEA 1 (green fluorescence) displayed a punctate distribution of early endosomes in P19 neuronal precursor cells (fig. 4.30). Synaptobrevin 2 (red fluorescence) was predominantly seen in the perinuclear compartment and did not localize to early endosomes. 

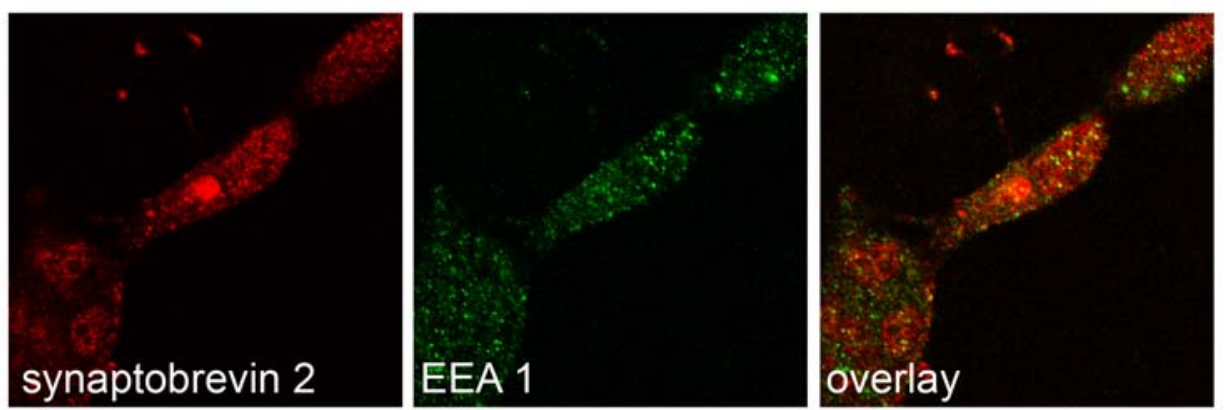

Fig. 4.30: Synaptobrevin 2 does not localize to early endosomes in P19 neuronal precursor cells. P19 neuronal precursors day 7 were labelled against synaptobrevin 2 (red) and EEA 1 (green) after fixation.

Since late recycling endosomes also locate to a perinuclear region in the cell the question was addressed if the perinuclear synaptic vesicle containing compartment might be part of the late endosomal system. Therefore colocalization of synaptobrevin 2 (red) with rab 11 (green) a small GTPase which is a marker for late recycling endosomes was analyzed (fig. 4.31).
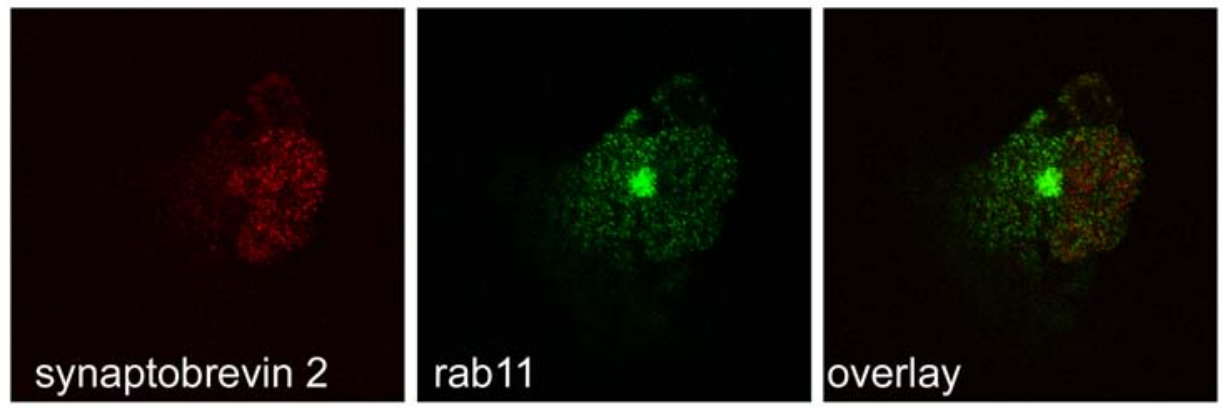

Fig. 4.31: Synaptobrevin 2 does not localize to a late recycling endosome in P19 neuronal precursor cells. P19 neuronal precursor cells d 7 were fixed and labelled against synaptobrevin 2 (red) and rab 11 (green).

The perinuclear compartment containing synaptic vesicle proteins did not show an overlapping distribution with the rab 11 labelled late endosome. Thus, we could not show if the perinuclear synaptobrevin 2 containing compartment belongs to the early or late endosomal sorting system.

In neuroendocrine cells synaptophysin is distributed between two vesicle populations of which one is also positive for the transferrin receptor suggesting an endosomal origin (Cameron et al. 1991, Povoda et al. 1999). At some point along the endocytic pathway synaptophysin has to be sorted away from the recycling transferrin receptor receptor positive compartment to synaptic vesicles.

These observations indicate a putative implication of the perinuclear compartment in recycling of synaptic vesicle proteins from the plasma membrane of neuronal precursor cells: When a vesicle fuses with the plasma membrane the luminal domain of a vesicle protein becomes exposed to the extracellular site. Internalization of the synaptic vesicle protein synaptotagmin 1 from the plasma membrane of P19 neurons was targeted by labelling its extracellular luminal domain with specific antibodies at 
$4{ }^{\circ} \mathrm{C}$. Upon high potassium stimulation and a temperature shift to $37{ }^{\circ} \mathrm{C}$ synaptotagmin 1 was endocytosed together with the antibodies from the plasma membrane. After fixatation cells were co-labelled for internalized synaptotagmin 1 (green) and synaptobrevin 2 (red fluoresence) see fig. 4.32.
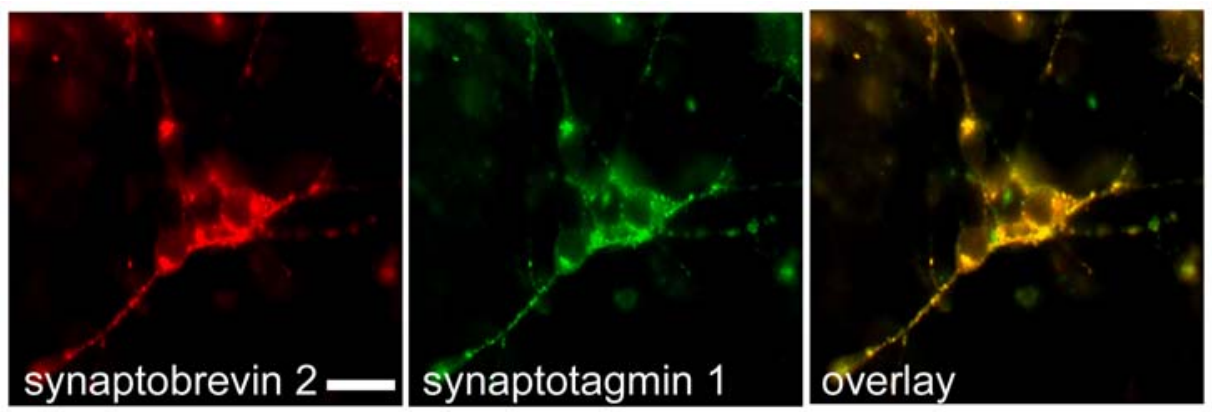

Fig. 4.32: Endocytosed synaptotagmin 1 is targeted to the perinuclear synaptobrevin 2 positive compartment in P19 neurons. P19 neurons were labelled with an antibody directed against the lumenal domain of synaptotagmin 1 at $4{ }^{\circ} \mathrm{C}$. The temperature was shifted to $37^{\circ} \mathrm{C}$ and internalization of the antibody was allowed for $30 \mathrm{~min}$. After fixation P19 neurons were labelled for endocytosed synaptotagmin 1 (green) and synaptobrevin 2 (red). Scalebar: $20 \mu \mathrm{m}$

In P19 neurons the synaptic vesicle protein synaptotagmin 1 was sorted from the plasma membrane to the perinuclear synaptobrevin 2 containing compartment. This observation supports a putative implication of the perinuclear compartment in the recycling of synaptic vesicle proteins. We hypothesize that cycling of synaptic vesicle proteins between the plasma membrane and the perinuclear compartment plays a role in the formation of a synaptic vesicle precursor organelle during neuronal differentiation of P19 cells.

Expression levels of endogenous synaptic vesicle proteins in P19 derived neurons were relatively low compared to primary neurons. Establishment of the antibody feeding assay as a tool to dissect recycling of synaptotagmin 1 during neuronal differentiation of P19 cells was the next aim of the study. But due to the low expression of endogenous synaptotagmin 1 in P19 neuronal precursor cells we have failed in the development of this assay as a quantitive tool to analyze synaptic vesicle protein recycling. 


\section{Discussion and outlook}

\subsubsection{The AMPA type glutamate receptor directly binds to the $\mu 2$-subunit of the clathrin-adaptor complex AP-2 via a dibasic sorting motif}

This work has focussed on clathrin-mediated endocytosis as a common mechanism of protein sorting at both sides of the synapse. At the postsynaptic site CME of AMPA-type glutamate receptors plays a role in the induction of hippocampal LTD by reducing the number of postsynaptic receptors available for signal transmission (Lee et al., 2002). CME requires recognition of cargo by the clathrin-adaptor AP-2 via endocytic sorting motifs comprised of a short linear array of amino acids. Basic sorting motifs represent a non-classical type of endocytic recognition motif in contrast to tyrosine- or dileucine based signals. Their implication in clathrin-mediated endocytosis has previously been characterized for various plasma membrane proteins. Best characterized is the interaction between the synaptic vesicle protein synaptotagmin 1 and the $\mu 2$ subunit of the clathrin-adaptor AP-2 (Chapman et al., 1998; Haucke et al., 2000). Recent work of Lee et al. (2002) has demonstrated that a basic cluster within the cytoplasmic tail of the postsynaptic AMPA receptor subunit GluR2 is critical for association with the AP-2 complex.

We have demonstrated here that the dibasic cluster within the GluR2 cytoplasmic tail links the receptor to the endocytic machinery by directly binding to the AP-2 complex via a mechanism similar to the recognition of the presynaptic protein synaptotagmin 1 by the $\mu 2$-subunit of AP-2.

This conclusion is based on several lines of evidence:

First, we could demonstrate direct binding of the GluR2 cytoplasmic tail to $\mu 2$ adaptin in pulldown experiments using in-vitro transcribed / translated or recombinantly expressed soluble $\mu 2$-adaptin. Recognition of $\mu 2$-adaptin by the AMPA receptor was critically dependent on a dibasic sorting motif within the membrane proximal portion of the GluR2 cytoplasmic tail.

Second, all AMPA receptor subunit cytoplasmic tails that harbour a basic recognition motif also directly bind to $\mu 2$-adaptin in biochemical experiments. Studies on AMPA receptor internalization in heterologeous cell lines or primary hippocampal neurons clearly show that GluR2 is the key player in agonist induced endocytosis of AMPARs (Man et al., 2000; Lee et al., 2000; 2004). Since AMPA receptors are heteromers basic recognition signals within the cytoplasmic tails of GluR1 or GluR3 might serve to enhance binding affinity of the multimeric receptor to $\mu 2$-adaptin. This notion is supported by work of Grass et al. (2004) showing that multimerization of synaptotagmin 1 increases binding affinity to the $\mu$-subunit of AP-2.

GluR2 is the only AMPA receptor subunit that in addition to AP-2 also binds to NSF (Nishimune et al., 1998; Lee et al., 2002). The binding sites for NSF and AP-2 within the membrane proximal portion of the AMPA receptor cytoplasmic tail are overlapping but not exactly identical (Lee et al., 2002). We found that an alanine K844A mutant of the GluR2 cytoplasmic tail is impaired in binding to $\mu 2-$ adaptin but still able to interact with NSF. This is in contrast to findings of Lee et al. (2002) showing that mutation of the basic cluster in GluR2 K844A disrupts binding to recombinantly expressed and purified NSF.

Third, a mutagenesis screen revealed that binding of soluble $\mu 2 \Delta 157$ to GluR2 is critically dependent on three basic residues within the cytoplasmic tail: 
mutation of each $\mathrm{K} 844$, R845 or K847 to alanines disrupted the interaction. Thus, basic residues within the cytoplasmic domain of a plasma membrane protein are sufficient to mediate recognition by the AP-2 complex. The binding mechanism of GluR2 to AP-2 via a basic cluster is in analogy to recognition of the presynaptic protein synaptotagmin 1 by $\mu 2$-adaptin via a dibasic cluster comprised within its C2B domain (Chapman et al., 1998).

Fourth, a synthetic peptide derived from the basic synaptotagmin 1 sorting motif competes binding of native AP-2 complex or recombinant $\mu 2$-adaptin to GluR2 indicating that the AMPA receptor and the synaptotagmin 1 basic motif occupy an identical binding site on $\mu 2$-adaptin. Furthermore, the synaptotagmin 1 basic peptide also blocked binding of native AP-2 to the inhibitory GABA A receptor subunit $\gamma 2$ intracellular loop that harbours a basic recognition motif for $\mu 2$. Basic sorting motifs have been recently described in a number of proteins. Apart from the postsynaptic AMPA and $\mathrm{GABA}_{\mathrm{A}}$ receptors and the presynaptic protein synaptotagmin 1 they are present in the polymeric immunoglobulin receptor, in the $\alpha_{1 \mathrm{~B}}$-adrenergic receptor, in the Nav1.2 sodium channel and in endoplasmic reticulum-Golgi intermediate compartment protein 53 (ERGIC 53) (Lee et al., 2002; Kittler, submitted; Aoreti et al., 1994; Diviani et al., 2003; Fache et al., 2004; Kappeler et al., 1994).

Results from this work imply that there is a common mechanism for the recognition of basic sorting motifs within a variety of cargo proteins by the clathrin-adaptor AP-2 via its $\mu 2$-subunit. Recent work from Grass et al. (2004) shows that affinity of AP-2 for synaptotagmin 1 is enhanced upon its multimerization. Since all known proteins comprising a basic recognition motif for $\mu 2$-adaptin are present as multimers at the plasma membrane it is reasonable to assume that basic sorting motifs serve as a link between multimeric plasma membrane proteins and the endocytic machinery in clathrinmediated internalization.

\subsubsection{A putative mechanism for the recognition of basic sorting motifs by $\mu 2$-adaptin}

The recognition for basic sorting motifs in $\mu 2$-adaptin is distinct from the binding site for canonical endocytic motifs such as tyrosine- or dileucine-based signals. Tyrosine based motifs bind to a hydrophobic pocket within $\mu 2$-subdomain $A$ (Owen et al., 1999). Diviani et al. (2003) located the binding site for a stretch of eight arginines within the $\alpha_{1 B}$-adrenergic receptor cytoplasmic tail to the $\mu 2$ subdomain $\mathrm{B}$. The synaptotagmin $1 \mathrm{C} 2 \mathrm{~B}$ domain also interacts with subdomain $B$ of $\mu 2$-adaptin (Haucke et al., 2000). Based on the electron charge density two acidic patches on the surface of the $\mu 2$-subomain $B$ surface, that represent putative recognition site for basic sorting motifs, were mutated (see fig. 5.1). A sequence alignment revealed that these acidic patches are specific for $\mu 2$ adaptin and that they are not conserved within the medium chain of other heterotetrameric adaptors such as AP-1, AP-3 or AP-4. 

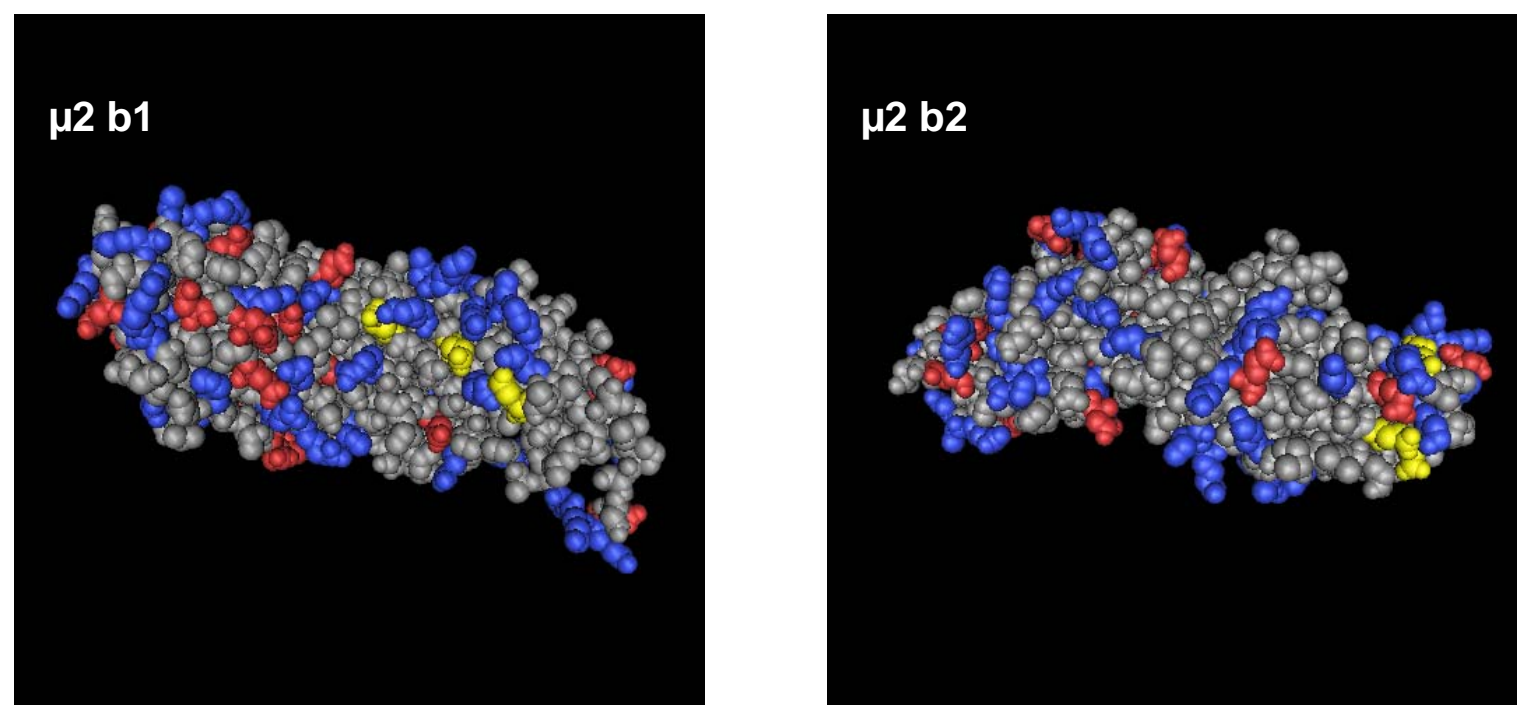

Fig. 5.1: The two putative recognition sites for basic sorting motif on the subdomain $B$ b1 or b2 are located to two acidic patches on opposite sides of the molecule. Charge density map of $\mu 2$-adaptin. Basic residues are labelled in blue whereas acidic residues are labelled in red. The putative binding sites for basic sorting motif b1 (E320, E348, E391) or b2 (E295, E369, E371) are labelled in yellow and locate to opposite sides of the molecule. Structure taken from Owen et al., 1999.

Mutation of the putative binding sites to alanines did not disrupt the interaction between the AMPA receptor cytoplasmic tail and $\mu 2$ in pulldown experiments (our unpublished data). Thus, these two acidic patches on the $\mu 2$-subdomain $B$ surface do not appear to function as binding sites for basic sorting motifs. From the present data one can conclude that the $\mu 2$-subdomain $B$ must harbour a specific recognition site for basic sorting motifs and that binding may require ionic interactions between the basic sorting motif and an acidic site on $\mu 2$.

A random based mutagenesis screen within the subdomain $B$ of $\mu 2$-adaptin could be used in the future for the characterization of the recognition site for basic endocytic motifs within $\mu 2$. Alternatively, a yeast-two-hybrid screen might provide another valuable tool to analyze the interaction between $\mu 2$-mutants and basic internalization motifs.

This method has the drawback that it does not give a structural explanation for the recognition mechanism of basic sorting motifs by $\mu 2$-adaptin. Cocrystallization of the basic peptide together with $\mu 2$-adaptin could reveal the structural basis for the recognition of basic sorting motifs and might thereby provide a detailed description of the molecular interaction mechanism.

Solid phase NMR analysis of the interaction could provide an alternative tool to gain structural insights into the recognition mechanism. 


\subsubsection{Interaction between the AMPA receptor cytoplasmic tail and $\mu 2$-adaptin may facilitate clathrin-mediated endocytosis of the receptor in-vivo}

We have provided evidence that the AMPA receptor subunit GluR2 interacts directly with the $\mu 2$-subunit of the AP-2 adaptor complex via a non-canonical sorting motif comprised of a dibasic cluster. Antibody internalization assays demonstrate that endocytosis of a synaptotagmin 1 chimeric construct expressed in a heterogeneous context is strongly dependent on two basic residues within the $\mu 2$-recognition motif of GluR2. However, mutation of the dibasic cluster into alanines did not completely disrupt its internalization from the plasma membrane of transfected cells: The cells were allowed to undergo endocytosis of the various constructs for 30 minutes which might promote unspecific background internalization of the construct by bulk endocytosis The quantification shows a clear decrease in internalization of the mutant synaptotagmin 1 chimeric construct and the truncated synaptotagmin 1 in transfected cells.

Thus, the dibasic $\mu 2$-recognition motif within the GluR2 cytoplasmic tail is sufficient to promote internalization of a synaptotagmin 1 chimeric construct from the plasma membrane of transfected cells. This result supports recent work by Grass et al. (2004) demonstrating that a dibasic sorting motif within the synaptotagmin $1 \mathrm{C} 2 \mathrm{~B}$ domain drives internalization of a chimeric construct when expressed in a heterologous context.

Internalization of the chimera is dependent both on the basic AP-2 binding sequence and functional AP-2 heterotetramers. Depletion of $\mu 2$-adaptin by small interfering RNA oligonucleotides specifically inhibits clathrin-mediated endocytosis in transfected cells (Motley et al., 2003; Hinrichsen et al., 2003). Using this tool to deplete $\mu 2$-adaptin the direct interaction partner of the GluR2 cytoplasmic tail we could interfere with internalization of the synaptotagmin 1 chimeric construct carrying the GluR2 basic sorting motif. The degree of internalization was similar to that of mutant synaptotagmin 1 chimeric construct or truncated synaptotagmin 1 . This result clearly demonstrates that a short basic motif derived from the AMPA receptor cytoplasmic tail is sufficient to target clathrin-mediated endocytosis of a receptor chimera by direct interaction with the $\mu 2$-subunit of the AP-2 complex in-vivo.

In-vivo experiments prove the existence of this interaction between $\mu 2$-adaptin and the dibasic cluster and demonstrate its implication in clathrin-mediated endocytosis of the AMPA receptor.

We believe that our data are of physiological relevance for the endocytic internalization of native AMPARs for several reasons:

First, disruption of the interaction between the AMPA receptor cytoplasmic tail and the AP-2 complex by mutating a lysine into an arginine prevents induction of hippocampal longterm depression (Lee et al., 2002) demonstrating a physiological implication of the interaction between GluR2 and the AP-2 complex in synaptic plasticity.

Second, our data on AMPA receptor internalization are supported by work from Kittler et al., (submitted) on characterization of the interaction between the inhibitory $\mathrm{GABA}_{\mathrm{A}}$ receptor subunits $\beta 3$ and $\gamma 2$ with $\mu 2$-adaptin.

Third, the synaptotagmin 1 derived basic sorting motif drives internalization of a chimeric construct from the plasma membrane of transfected cells suggesting a 
role for the basic motif in clathrin-mediated recycling of synaptotagmin 1 at the presynaptic terminal (Grass et al., 2004).

Thus, the recognition of basic sorting motifs by $\mu 2$-adaptin serves as a common link to the endocytic machinery in the internalization of proteins from both sides of the synapse (Chapman et al., 1998; Haucke et al., 2000).

A screen for basic sorting motifs within other multimeric plasma membrane proteins might reveal a role for basic recognition motifs in other physiological processes that require highly regulated uptake of proteins by clathrin-mediated endocytosis.

Internalization of AMPA receptors in NMDA stimulated longterm depression is predominantly regulated by protein interactions with the GluR2 cytoplasmic tail and covalent modifications including phosphorylation and perhaps ubiquitination (Bredt and Nicoll, 2003). Work of Lee et al. (2002) shows that interaction between the GluR2 cytoplasmic tail and AP-2 is specifically involved in NMDA induced but not AMPA induced internalization of AMPA receptors from the glutamatergic synapse. Thus, it would be very interesting to characterize the role of $\mu 2$-binding to the GluR2 cytoplasmic tail in the context of other protein interactions involved in NMDA induced LTD. AP-2 and NSF share an overlapping recognition site in the membrane proximal portion of the GluR2 cytoplasmic tail (Nishimune et al., 1998; Lee et al., 2002). Interaction of GluR2 with NSF is thought to stabilize AMPARs at the synapse, whereas binding to the clathrin-adaptor AP-2 facilitates receptor endocytosis in NMDA induced hippocampal LTD (Lee et al., 2002). Endocytosis in dendrites occurs at specialized endocytic zones adjacent to the postsynaptic density (Blanpied et al., 2002) suggesting that interaction of the GluR2 cytoplasmic tail with either NSF or AP-2 might underlie a spatial regulation depending on synaptic or extra synaptic localization of the receptor.

Covalent modification of the AMPA receptor cytoplasmic tail by tyrosine phosphorylation is involved in agonist stimulated internalization of the receptor (Hayashi et al., 2004; Hamada et al., 2004). Serine phosphorylation of the GluR2 cytoplasmic tail regulates its interaction with the PDZ binding protein PICK 1 and thereby facilitates induction of LTD (Chung et al., 2000; Trachoma et al., 2004; Gardner et al., 2005). Ubiquitination of the AMPA receptor cytoplasmic tail in C. elegant (Bureau et al., 2002) or of the postsynaptic scaffolding protein PSD 95 (College et al., 2003) provides an alternative type of covalent modification that regulates surface density of AMPA receptors. Based on these findings it might be an issue to analyze if covalent modification of the GluR2 cytoplasmic tail either by phosphorylation or ubiquitination affects binding to the AP-2 complex and thereby regulates clathrin-mediated endocytosis of the receptor.

Taken together results from this work imply a role for direct binding of the AMPA receptor cytoplasmic tail to the AP-2 complex in clathrin-mediated endocytosis of the receptor during LTD. Thus, CME serves as a mechanism to regulate the density of postsynaptic receptors in synaptic plasticity. A basic sorting motif within the GluR2 cytoplasmic tail serves as a link to the endocytic machinery via the clathrin-adaptor AP-2. At the presynaptic site a basic sorting motif promotes CME of synaptotagmin 1 and thereby facilitates synaptic vesicle recycling.

Collectively, our findings imply the hypothesis that basic sorting motifs function in the regulated uptake of multimeric proteins from the postsynaptic as well as the presynaptic active zone. 


\subsubsection{A functional model for the role of the interaction between the AMPA receptor and the clathrin-adaptor AP-2 in synaptic plasticity}

In contrast to the establishment of LTP that increases the number of postsynaptic AMPARs, the induction of LTD includes a reduction of synaptic AMPARs and their subsequent internalization from the plasma membrane. The AMPA receptor subunit GluR2 has been shown to be the key player in regulated uptake of AMPARs upon agonist stimulation (Lin et al., 2000; Man et al., 2000; Lee et al., 2002). All AMPA receptor subunits harbour a basic recognition site for $\mu 2$-adaptin within their cytoplasmic tail. But only the GluR2 subunit contains a binding site for the hexametric Atlases NSF that is overlapping with the basic $\mu 2$-recognition motif (Lee et al., 2002). Thus, differential interaction of the GluR2 cytoplasmic tail with either NSF or AP-2 might regulate the synaptic expression of AMPA receptors in neuronal plasticity (see fig. 5.2). Binding of GluR2 to NSF in conjunction with binding to the PDZ domain protein GRIP might function in the stabilization of synaptic AMPA receptors (Bredt and Nicholls, 2003). Since, internalization of AMPA receptors occurs predominantly at specialized endocytic site in the dendrite spine adjacent to the postsynaptic density (Blanpied et al., 2002). AMPA receptors have to be undeterred from the postsynaptic scaffold before they can diffuse laterally to the extra synaptic plasma membrane where endocytosis takes place (Bredt \& Nicoll, 2003; Bergdorf \& Coquet, 2002). Phosphorylation-dependent interaction of the GluR2 cytoplasmic tail with the PDZ domain protein PICK 1 is thought to be involved in this process (Bredt \& Nichol, 2003). At some point in this process NSF has to disassociate from the GluR2 cytoplasmic tail to release the basic motif for recognition by the endocytic machinery.

Up to now it is unknown which molecular mechanisms regulate the exchange of NSF for AP-2 and thereby facilitate clathrin-mediated endocytosis of the AMPA receptor in NMDA induced LTD (see fig. 5.2).

Thus, we propose that a hitherto unknown mechanism switches AMPAR cytoplasmic tail from NSF-mediated exocytose delivery to synaptic sites to clathrin- and AP-2 dependent internalization during longterm depression (LTD). Another major issue in the activity dependent endocytosis of AMPARs is how covalent modification of the GluR2 cytoplasmic tail either by phosphorylation or ubiquitination (Hamada et al., 2004; Hayashi \& Hogan, 2004; College et al., 2003; Bureau et al., 2002) regulates protein interactions during the induction of LTD. 


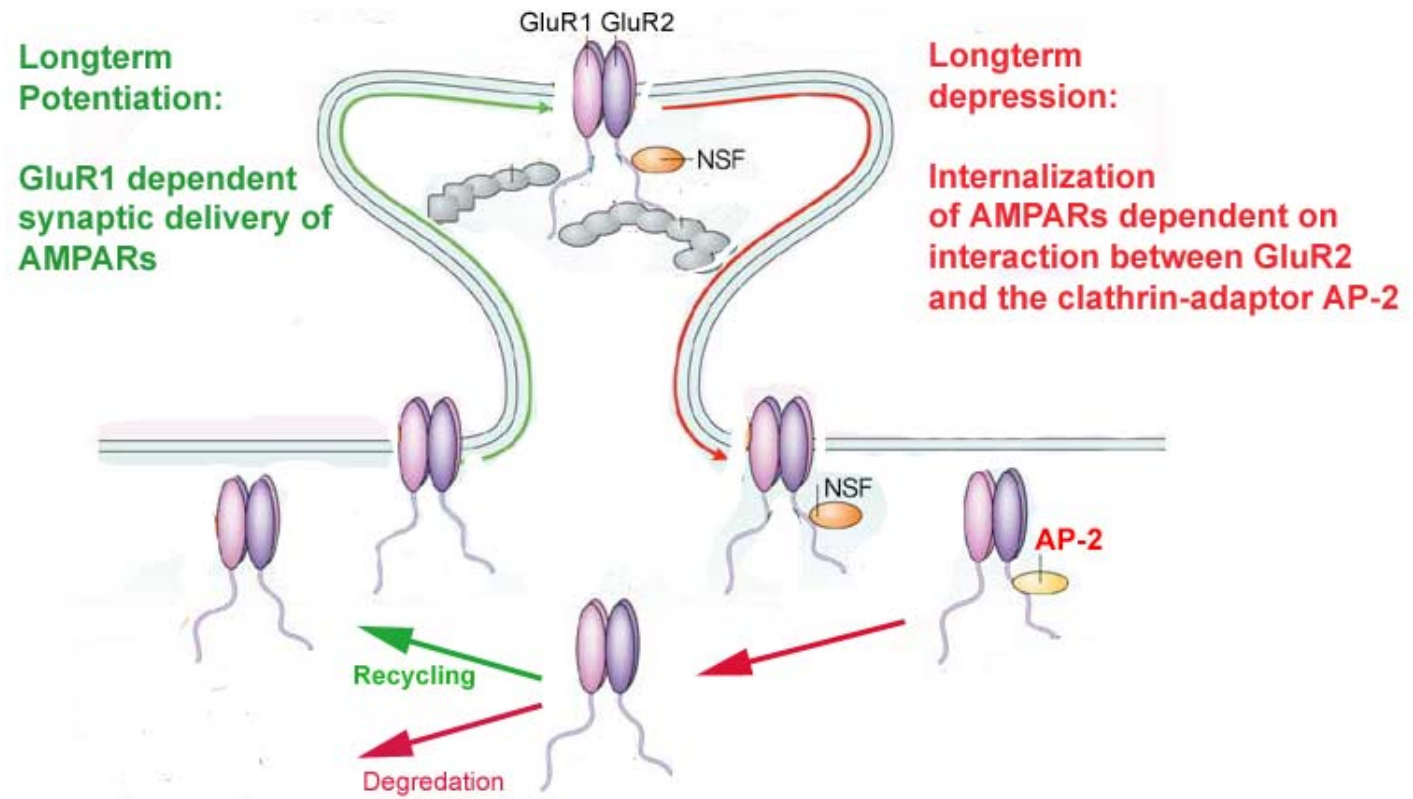

Fig. 5.2: Movement of AMPA receptors during neuronal plasticity. GluR1 is the key subunit that targets synaptic delivery of AMPA receptors during LTP. NMDA induced LTD requires clathrin-mediated endocytosis of AMPARs from the postsynaptic plasma membrane that is mediated by direct binding of a basic sorting motif within the GluR2 cytoplasmic tail to the $\mu 2$ subunit of the clathrin-adaptor complex AP-2. Internalized AMPARs can undergo activity dependent intracellular sorting steps that target either their liposome degradation or their recycling to the plasma membrane.

\subsubsection{Differential expression of synaptic vesicle proteins during neuronal differentiation may regulate de-novo synaptogenesis}

At the postsynaptic side clathrin-mediated endocytosis of neurotransmitter receptors is involved in synaptic plasticity, at the presynaptic side CME plays an important role in synaptic vesicle recycling. Studies on neuroendocrine cells implicate a putative role for endosomal sorting in the formation of synaptic vesicle like micro vesicles (Slavs) (Cameron et al., 1991; Provide et al., 1999). These finding arose the question if protein sorting by clathrin-mediated endocytosis provides a mechanism to establish axonal polarity during neuronal differentiation. We aimed to find out if recycling of SV proteins from the plasma membrane to a perinuclear presumably endosomal recycling compartment plays a role in the biogenesis of a putative synaptic vesicle precursor organelle.

Retinoic acid-induced neuronal differentiation of an embryo carcinoma cell line was used as a model system to study the de-novo formation of synaptic vesicles during neuronal differentiation. Primary hippocampal neurons provide a well established tool to study synaptogenesis in culture. They express synaptic 
vesicle proteins from very early stages on prior to neuritis outgrowth and synaptogenesis (Fletcher et al., 1994). This precludes the study of the de-novo biogenesis of SUVs. In contrast to primary hippocampal neurons pluripotent embryo carcinoma cells do not express Saps before induction of neuronal differentiation which was an important prerequisite to analyze SV biogenesis in cell-culture.

RT-PCR revealed that expression of genes encoding for Saps is switched on at different time-points during neuronal differentiation. The temporal coordination of SVP expression analyzed by immuno-cytochemistry confirmed the mRNA expression pattern. In analogy to the development of primary hippocampal neurons expression of SV proteins in P19 neuronal precursors is switched on at very early stages of differentiation prior to neurite outgrowth. SV proteins in these cells localize to a perinuclear compartment of unknown function and organellar origin that has not been described before.

Studies of Fletcher et al. (1994) on SVP expression during differentiation of primary hippocampal neurons support the notion that differential expression of SVPs plays a role in the regulation of synaptogenesis.

These observations imply the question if SVPs play an active role in the formation of the presynaptic compartment during neuronal differentiation. Another open question is how correct sorting of SVPs to the future axon is achieved during neurite outgrowth and axodendritic compartimentalization of a neuronal precursor cell. A third question concerns the molecular machinery that mediates directed transport of SVPs and other active zone components along the future axon to the nascent synapse. A last issue to be resolved is how intercellular communication between neurons or neurons and glial cells regulates de-novo synaptogenesis during neuronal communication.

\subsubsection{A putative role for clathrin-mediated endocytosis in synaptic vesicle biogenesis}

One major question addressed in this work is if endosomal sorting of SV proteins plays a role in the establishment of axonal polarity during neuronal differentiation. One possible mechanism of axonal targeting is direct transport of proteins from the trans-Golgi network to the future axon. Alternatively, axonal proteins may undergo indirect transport to the axonal compartment by being internalized from the somatodendritic plasma membrane via clathrin-mediated endocytosis and then being sorted to the axon from a recycling endosome.

Thus, the question arose if the perinuclear SVP containing compartment originated from the Golgi or whether it was part of the endosomal sorting machinery.

The perinuclear compartment does not form part of the Golgi-complex. Colocalization of internalized transferrin with synaptic vesicle markers in the perinuclear compartment hints at an endosomal origin. This view is supported by findings that in neuroendocrine PC 12 cells synaptophysin co-localizes with endocytic markers (Johnston et al., 1989; Cameron et al., 1991). However, synaptic vesicle proteins in the compartment do not co-localize with markers of the early or late endosomal system such as EEA 1 or Rab 11.

Thus, the exact organellar origin of the perinuclear SVP containing compartment in neuronal precursor cells has been an open question. 
Our data using differentiation of P19 cells and antibody feeding assays have demonstrated that endogenous synaptotagmin 1 is endocytosed from the plasma membrane and transported to the perinuclear compartment in postmitotic neurons.

This finding suggests a possible function of the compartment in recycling of SVPs. Our observations favour an indirect transport route of SV components to the future axon via recycling from the somatodendritic plasma membrane to a perinuclear compartment by clathrin-mediated endocytosis. Cycling of SVPS between the plasma membrane and the putative perinuclear recycling compartment might serve to separate axonal from somatodendritic proteins and give rise to a SV precursor organelle that is then transported to the forming synapse.

Studies on synaptogenesis in mature neurons imply that active zone components are transported to the forming synapse in distinct pre-assembled transport packets (Zhai et al., 2001; Ahmari et al., 2000). Active zone components including scaffolding proteins piccolo and bassoon and the plasma membrane SNARE proteins syntaxin and SNAP 25 are transported in dense core granulated vesicles devoid of SV components (Zhai et al., 2001). Work of Ahmari et al. (2000) has described discrete transport packets of heterogeneous morphology containing the synaptic vesicle SNARE protein synaptobrevin I VAMP 2 together with synaptotagmin 1 . These findings show that the presynaptic site is assembled from discrete prefabricated transport packets during synaptogenesis in mature hippocampal neurons.

In summary, at least two different active zone precursor organelles, one containing predominantly active zone elements and the other one synaptic vesicle components, have been described up to now (Zhai et al., 2001; Ahmari et al., 2000).

Our studies in agreement with findings in neuroendocrine cells (Cameron et al., 1991; Provoda et al., 2000) support the hypothesis that clathrin-mediated endocytosis of SVPs plays a role in formation of SV precursor organelles during neuronal differentiation.

\subsubsection{Targeted transport of active zone precursor vesicles to the forming synapse}

Synaptic vesicle components residing within the perinuclear compartment of neuronal precursor cells need to be correctly transported along the axon to the forming synapse. Axonal transport is achieved by microtubular dependent vesicular trafficking of proteins to the distinct specialized zone of the membrane (Goldstein \& Philp, 1998; Hirokawa, 1998).

Motorproteins of the kinesin superfamily have been shown to mediate differential transport of vesicles containing certain synaptic vesicle proteins: For example the monomeric KIF1A transports vesicles along microtubule that contain the SVPs synaptotagmin 1, synaptophysin and Rab3A but lack other SVPs such as SV 2 or components of the active zone exocytic machinery (Okada et al., 1995). Thus, KIF1A might be the motorprotein that functions in the transport of SVPs from the perinuclear compartment along the developing axon. Motor proteins that mediate transport of other active zone precursor vesicles containing i. e. piccolo and bassoon have not been identified up to now. 
Based on these observations it is reasonable to assume that differential transport of active zone precursor vesicles along the axon is mediated by a distinct set of specialized motorproteins.

Another open question concerns cargo recognition signals in axonal versus somatodendritic transport. SVPs within the perinuclear compartment differ strongly in their structure and function suggesting that they do not share a universal recognition signal for axonal transport. This implies the question if SVPs are transported to the axon in an SV precursor organelle or if each protein is differentially sorted to the future axon.

Apical targeting, that would correspond to axonal targeting in neurons, in polarized epithelial cells is often controlled by extracellular carbohydrate determinants (Winckler \& Mellman, 1999). Many synaptic vesicle proteins such as synaptotagmin 1 and SV2 are known to be highly glycosylated (Han et al., 2004). Thus, specific glycosylation might mediate axonal sorting of SVPs from the perinuclear compartment to the forming synapse during neuronal differentiation.

Implication of 'lipid rafts' comprised of sphingomyelin and cholesterole as a platform for axonal sorting was demonstrated by work on the axonal targeting of Thy 1(Ledesma et al., 1999; Winckler \& Mellman, 1999). The synaptic vesicle protein synaptophysin 1 directly interacts with cholesterole (Thiele et al., 2000).

These findings suggest, that formation of lipid microdomains is involved in the establishment of an axonal sorting machinery during neuronal differentiation.

Analysis of the lipid composition of the SVP containing perinuclear organelle might shed light on the role of protein-lipid interactions in the formation of SV precursor organelles.

In immature embryonic stem cell derived neuronal precursor SVPs such as synaptotagmin 1 are present in all outgrowing processes of the cell. Their distribution is not confined to the future axon (own observations). These observations are supported by the finding that correct axonal targeting of Thy 1 is developmentally regulated during neuronal differentiation (Ledesma et al., 1999). Thus, establishment of a 'lipid raft' based sorting machinery for axonal proteins during neuronal differentiation plays an important role in the formation of the presynaptic compartment.

It is tempting to speculate that differential lipid compositions in specialized active zone precursor organelles play a role in the correct sorting of active zone elements into the corresponding transport packet. For example, the SVP synaptophysin has been shown to interact directly with cholesterole (Thiele et al., 2000). Synaptophysin forms a complex with synaptobrevin / VAMP 2 that is developmentally upregulated (Becher et al., 1999). Thus, dependent on its interaction with cholesterole synaptophysin might mediate the sorting of synaptobrevin / VAMP 2 and other synaptic vesicle components i. e. synaptotagmin 1 (Ahmari et al., 2000) into a SV precursor organelle that is then transported to the nascent synapse. Such an organelle might be formed from the perinuclear synaptic vesicle protein containing compartment described in this work. 


\subsubsection{A putative role for clathrin-mediated endocytosis of SV proteins at different stages of synaptogenesis}

Up to now the spatial regulation of SV formation remains an open issue. Observations of Ahmari et al. (2000) have demonstrated that SVPs are transported along the axon in morphological heterogeneous vesicles that are not reminiscent of round electron lucent $50 \mathrm{~nm}$ synaptic vesicles. Based on this observation the question arises if SVs are directly formed from the perinuclear compartment or if SV formation from pre-assembled precursor organelles takes place at the forming synapse.

Clathrin-mediated endocytosis is a major event in the retrieval of excess membrane and SVPs after exocytosis (Heuser \& Reese, 1973). Therefore, clathrin-mediated recycling of SV components may also play a role in the formation of synaptic vesicles from a precursor organelle.

How SV precursor organelles are trapped at the site of the forming synapse is another open question.

The interaction of postsynaptic neuroligins with presynaptically expressed $\beta$ neurexins promotes clustering of synaptic vesicles during neuronal differentiation (Scheiffele et al., 2000; Dean et al., 2003) possibly by direct interaction between the SVP synaptotagmin 1 with the cytoplasmic tail of $\beta$ neurexin (Butz et al., 1998).

This observation implies the hypothesis that different SV proteins within an SV precursor organelle fulfil different roles: Synaptophysin possibly mediates formation of the SV precursor organelle from the endosomal system whereas synaptotagmin 1 might be involved in axonal targeting of the organelle to the forming synapse.

Clathrin-mediated endocytosis may be involved at two distinct stages of synaptogenesis. In a first step clathrin-dependent cycling of SVPs between the plasma membrane and a perinuclear endosomal compartment might give rise to a SV precursor organelle that is transported along the axon. At the forming synapse CME might be involved in the formation of synaptic vesicles from a precursor organelle.

\subsubsection{Retinoic acid-induced neuronal differentiation of embryonic precursor cells as a model system to characterize the de-novo SV biogenesis}

In this work we have characterized the retinoic acid induced differentiation of embryonic precursor cells into postmitotic neurons as a tool to study the denovo biogenesis of synaptic vesicles. Our findings indicate that a perinuclear SVP containing compartment in neuronal precursor cells plays a role in the formation of a SV precursor organelle.

The very low expression levels of SVPs in neuronal precursor cells represented a drawback in the establishment of a functional endocytosis based assay to characterize a putative recycling function of the perinuclear compartment.

One possibility to overcome this obstacle would be the use of an embryonic stem cell line stably expressing a GFP-tagged synaptobrevin / VAMP 2 described in the work of Ahmari et al. (2000) under the control of an inducible promotor. Retinoic acid-induced differentiation of this cell line should the provide 
insight into the intracellular distribution of synaptic vesicle proteins (SVPS) during neuronal differentiation. An antibody feeding assay addressing the GFPtagged synaptobrevin 2 could be used to analyze a putative function of the perinuclear compartment in the recycling of SVPs from the plasma membrane of neuronal precursor cells.

To test the hypothesis, that clathrin-mediated cycling of SVPs between the plasma membrane and the perinuclear compartment might be involved in the formation of a SV precursor organelle during neuronal differentiation, one could use siRNA interference directed against clathrin or $\mu 2$-adaptin (Motley et al., 2003; Hinrichsen et al., 2003) to deplete CME.

Several lines of evidence indicate that protein-lipid interactions play a role in the establishment of an axonal sorting machinery (Ledesma et al., 1999; Thiele et al., 2000; Mauch et al., 2001). Immuno-isolation of the perinuclear SV containing compartment and analysis of its lipid composition at different stages of neuronal development might give information on the regulation of protein-lipid interactions in the formation of a SV precursor organelle.

To address the question if SV proteins are involved at different steps in the formation and transport of a SV precursor organelle siRNA interference directed against SVPs in neuronal precursor cells could be used as a tool. Depletion of synaptophysin would disrupt the formation of the developmentally upregulated complex with synaptobrevin / VAMP 2 (Becher et al., 1999) and should thereby give information on the role of this complex in the formation of an SV precursor organelle. By knocking-down synaptotagmin 1 in neuronal precursors one could possibly interfere with the axonal transport of the SV precursor organelle. 


\section{Summary and conclusions}

Internalization of proteins from the synaptic plasma membrane by means of a clathrin-coated endocytosis is involved in the regulation of signal transmission a both sides of the synapse. In this work we have addressed the question if a dibasic AP-2 recognition motif links proteins from both sides of the synapse directly to the endocytic machinery.

Our biochemical data demonstrate that recognition of the AMPA-type glutamate receptor subunit GluR2 by the $\mu 2$-subunit of AP-2 complex is critically dependent on a dibasic cluster within the GluR2 cytoplasmic tail. Binding occurs by a mechanism similar to the recognition of the presynaptic protein synaptotagmin 1 by $\mu 2$-adaptin via a basic sorting motif. Competition experiments using a synthetic peptide based on the synaptotagmin 1 sorting motif have revealed that the GluR2 and the synaptotagmin 1 basic motif occupy an identical binding site on the surface of $\mu 2$-adaptin. A basic stretch within an intracellular loop also mediates binding of the $G_{A B A} A^{-}$ receptor subunit $\gamma 2$ to $\mu 2$-adaptin which can be competed by the synaptotagmin 1 derived synthetic peptide. These findings imply that recognition of synaptic proteins via dibasic clusters within their cytoplasmic portion is involved in clathrin- and AP-2 dependent internalization at both sides of the synapse.

Using a site directed mutagenesis approach we have aimed to characterize two acidic patches on opposite faces of the $\mu 2$ subdomain $B$ as putative recognition sites for basic motifs. But insertion of the mutations did not affect binding of $\mu 2$-adaptin to GluR2.

AP-2 dependent internalization of AMPARs from the postsynaptic plasma membrane is involved in agonist induced synaptic plasticity at the postsynaptic side. Using a chimeric approach we wanted to see if recognition of the dibasic AMPA receptor sorting motif by $\mu 2$-adaptin provides a direct link to the endocytic machinery. The dibasic cluster facilitated internalization of a synaptotagmin 1 chimera carrying the AMPA receptor sorting motif from the plasma membrane of transfected cells. Depletion of the interaction between $\mu 2$-adaptin and the dibasic recognition motif in transfected cells by siRNAs directed against $\mu 2$-adaptin resulted in a decrease of clathrin-mediated endocytosis of the construct.

Thus, internalization of native AMPA receptors from the postsynaptic plasma membrane during longterm depression (LTD) is both dependent on the basic recognition motif within the GluR2 cytoplasmic tail and intact heterotetramers of the clathrin-adaptor

AP-2. Our data support the hypothesis that that dibasic AP-2 recognition motifs play a role in clathrin-mediated endocytosis of membrane cargo proteins from the plasma lemma at both sides of the synapse.

Upon induction of longterm potentiation (LTP) new presynaptic active zones are assembled from pre-assembled transport packets containing active zone elements or synaptic vesicle (SV) components. We have aimed to shed light on the de-novo biogenesis of SVs during 
the establishment of axonal polarity. We have established the retinoic acid-induced neuronal differentiation of unpolarized embryonic precursor cells as a model system to study de-novo synaptogenesis. RT-PCR has demonstrated that expression of mRNAs encoding for SVPs is initiated at different stages of neuronal development. Some SVPs such as the vesicular SNARE protein synaptobrevin / VAMP 2 were expressed from very early differentiation stages on that preceded neurite outgrowth and synaptogenesis. In neuronal precursor cells SVPs have been found to localize to a perinuclear compartment that might represent a SV precursor organelle.

Brefeldin A treatment of neuronal precursor cells has revealed that the perinuclear SVP containing compartment does not originate from the Golgi-apparatus. To test the hypothesis that a SV precursor organelle might originate from the endosomal system we have analyzed co-localization of the perinuclear SVP containing compartment with markers for early or late endosomes. We could not observe co-localization. However, functional assays have indicated an endosomal origin of the perinuclear SVP containing compartment: Endocytosed transferrin was transported to the perinuclear SVP containing compartment where it co-localized with endogenous synaptobrevin 2 . This finding implies a putative function as a recycling compartment for SVPs. An antibody feeding assay in postmitotic neurons has shown that a antibodies directed against the luminal domain of the SVP synaptotagmin 1 were sorted to the perinuclear SVP containing compartment after endocytosis from the plasma membrane. Since the expression levels of SVPs in neuronal precursor cells were very low we have failed in the attempt to establish the antibody feeding assay as a tool to analyze the recycling of SVPs during neuronal differentiation.

Thus, we have developed and initially characterized a model system for neuronal differentiation based embryonic stem cells. 


\section{Bibliography}

Ahmadian, G., Ju, W., Liu, L., Wyszynski, M., Lee, S. H., Dunah, A. W., Taghibiglou, C., Wang, Y., Lu, J., Wong, T. P., et al. (2004). Tyrosine phosphorylation of GluR2 is required for insulin-stimulated AMPA receptor endocytosis and LTD. Embo J 23, 1040-1050.

Ahmari, S. E., Buchanan, J., and Smith, S. J. (2000). Assembly of presynaptic active zones from cytoplasmic transport packets. Nat Neurosci 3, 445-451.

Ahmari, S. E., and Smith, S. J. (2002). Knowing a nascent synapse when you see it. Neuron 34, 333-336.

Ashby, M. C., De La Rue, S. A., Ralph, G. S., Uney, J., Collingridge, G. L., and Henley, J. M. (2004). Removal of AMPA receptors (AMPARs) from synapses is preceded by transient endocytosis of extrasynaptic AMPARs. J Neurosci 24, 5172-5176.

Beattie, E. C., Carroll, R. C., Yu, X., Morishita, W., Yasuda, H., von Zastrow, M., and Malenka, R. C. (2000). Regulation of AMPA receptor endocytosis by a signaling mechanism shared with LTD. Nat Neurosci 3, 1291-1300.

Becher, A., Drenckhahn, A., Pahner, I., Margittai, M., Jahn, R., and Ahnert-Hilger, G. (1999). The synaptophysin-synaptobrevin complex: a hallmark of synaptic vesicle maturation. J Neurosci 19, 1922-1931.

Blanpied, T. A., Scott, D. B., and Ehlers, M. D. (2002). Dynamics and regulation of clathrin coats at specialized endocytic zones of dendrites and spines. Neuron 36, 435-449.

Bloom, O., Evergren, E., Tomilin, N., Kjaerulff, O., Low, P., Brodin, L., Pieribone, V. A., Greengard, P., and Shupliakov, O. (2003). Colocalization of synapsin and actin during synaptic vesicle recycling. J Cell Biol 161, 737-747.

Bonifacino, J. S., and Traub, L. M. (2003). Signals for sorting of transmembrane proteins to endosomes and lysosomes. Annu Rev Biochem 72, 395-447.

Bradke, F., and Dotti, C. G. (2000). Establishment of neuronal polarity: lessons from cultured hippocampal neurons. Curr Opin Neurobiol 10, 574-581.

Bredt, D. S., and Nicoll, R. A. (2003). AMPA receptor trafficking at excitatory synapses. Neuron 40, 361-379. 
Burbea, M., Dreier, L., Dittman, J. S., Grunwald, M. E., and Kaplan, J. M. (2002). Ubiquitin and AP180 regulate the abundance of GLR-1 glutamate receptors at postsynaptic elements in C. elegans. Neuron 35, 107-120.

Cameron, P. L., Sudhof, T. C., Jahn, R., and De Camilli, P. (1991). Colocalization of synaptophysin with transferrin receptors: implications for synaptic vesicle biogenesis. J Cell Biol 115, 151-164.

Carroll, R. C., Beattie, E. C., von Zastrow, M., and Malenka, R. C. (2001). Role of AMPA receptor endocytosis in synaptic plasticity. Nat Rev Neurosci 2, 315-324.

Carroll, R. C., Beattie, E. C., Xia, H., Luscher, C., Altschuler, Y., Nicoll, R. A., Malenka, R. C., and von Zastrow, M. (1999). Dynamindependent endocytosis of ionotropic glutamate receptors. Proc Natl Acad Sci U S A 96, 14112-14117.

Chapman, E. R., Desai, R. C., Davis, A. F., and Tornehl, C. K. (1998). Delineation of the oligomerization, AP-2 binding, and synprint binding region of the $\mathrm{C} 2 \mathrm{~B}$ domain of synaptotagmin. J Biol Chem 273, 32966-32972.

Chung, H. J., Xia, J., Scannevin, R. H., Zhang, X., and Huganir, R. L. (2000). Phosphorylation of the AMPA receptor subunit GluR2 differentially regulates its interaction with PDZ domain-containing proteins. J Neurosci 20, 7258-7267.

Cohen-Cory, S. (2002). The developing synapse: construction and modulation of synaptic structures and circuits. Science 298, 770-776.

Colledge, M., Snyder, E. M., Crozier, R. A., Soderling, J. A., Jin, Y., Langeberg, L. K., Lu, H., Bear, M. F., and Scott, J. D. (2003). Ubiquitination regulates PSD-95 degradation and AMPA receptor surface expression. Neuron 40, 595-607.

Collingridge, G. L., Isaac, J. T., and Wang, Y. T. (2004). Receptor trafficking and synaptic plasticity. Nat Rev Neurosci 5, 952-962.

Daly, C., and Ziff, E. B. (1997). Post-transcriptional regulation of synaptic vesicle protein expression and the developmental control of synaptic vesicle formation. J Neurosci 17, 2365-2375.

Diviani, D., Lattion, A. L., Abuin, L., Staub, O., and Cotecchia, S. (2003). The adaptor complex 2 directly interacts with the alpha $1 \mathrm{~b}$ adrenergic receptor and plays a role in receptor endocytosis. J Biol Chem 278, 19331-19340.

Dotti, C. G., Sullivan, C. A., and Banker, G. A. (1988). The establishment of polarity by hippocampal neurons in culture. $\mathrm{J}$ Neurosci 8, 1454-1468. 
Ehrlich, M., Boll, W., Van Oijen, A., Hariharan, R., Chandran, K., Nibert, M. L., and Kirchhausen, T. (2004). Endocytosis by random initiation and stabilization of clathrin-coated pits. Cell 118, 591-605.

Feany, M. B., Yee, A. G., Delvy, M. L., and Buckley, K. M. (1993). The synaptic vesicle proteins SV2, synaptotagmin and synaptophysin are sorted to separate cellular compartments in $\mathrm{CHO}$ fibroblasts. J Cell Biol 123, 575-584.

Fletcher, T. L., De Camilli, P., and Banker, G. (1994). Synaptogenesis in hippocampal cultures: evidence indicating that axons and dendrites become competent to form synapses at different stages of neuronal development. J Neurosci 14, 6695-6706.

Fotin, A., Cheng, Y., Sliz, P., Grigorieff, N., Harrison, S. C., Kirchhausen, T., and Walz, T. (2004). Molecular model for a complete clathrin lattice from electron cryomicroscopy. Nature 432, 573-579.

Galli, T., and Haucke, V. (2004). Cycling of synaptic vesicles: how far? How fast! Sci STKE 2004, re19.

Gardner, S. M., Takamiya, K., Xia, J., Suh, J. G., Johnson, R., Yu, S., and Huganir, R. L. (2005). Calcium-permeable AMPA receptor plasticity is mediated by subunit-specific interactions with PICK1 and NSF. Neuron 45, 903-915.

Goda, Y., and Davis, G. W. (2003). Mechanisms of synapse assembly and disassembly. Neuron 40, 243-264.

Goritz, C., Mauch, D. H., Nagler, K., and Pfrieger, F. W. (2002). Role of glia-derived cholesterol in synaptogenesis: new revelations in the synapse-glia affair. J Physiol Paris 96, 257-263.

Grass, I., Thiel, S., Honing, S., and Haucke, V. (2004). Recognition of a basic AP-2 binding motif within the C2B domain of synaptotagmin is dependent on multimerization. J Biol Chem 279, 54872-54880.

Gundelfinger, E. D., Kessels, M. M., and Qualmann, B. (2003). Temporal and spatial coordination of exocytosis and endocytosis. Nat Rev Mol Cell Biol 4, 127-139.

Han, W., Rhee, J. S., Maximov, A., Lao, Y., Mashimo, T., Rosenmund, C., and Sudhof, T. C. (2004). N-glycosylation is essential for vesicular targeting of synaptotagmin 1 . Neuron 41, 8599. 
Haucke, V. (2000). Dissecting the ins and outs of excitement: glutamate receptors on the move. Nat Neurosci 3, 1230-1232.

Haucke, V., Wenk, M. R., Chapman, E. R., Farsad, K., and De Camilli, P. (2000). Dual interaction of synaptotagmin with mu2- and alpha-adaptin facilitates clathrin-coated pit nucleation. Embo $\mathrm{J} 19$, 6011-6019.

Hayashi, T., and Huganir, R. L. (2004). Tyrosine phosphorylation and regulation of the AMPA receptor by SRC family tyrosine kinases. J Neurosci 24, 6152-6160.

Herring, D., Huang, R., Singh, M., Robinson, L. C., Dillon, G. H., and Leidenheimer, N. J. (2003). Constitutive GABAA receptor endocytosis is dynamin-mediated and dependent on a dileucine AP2 adaptin-binding motif within the beta 2 subunit of the receptor. $\mathrm{J}$ Biol Chem 278, 24046-24052.

Heuser, J. E., and Reese, T. S. (1973). Evidence for recycling of synaptic vesicle membrane during transmitter release at the frog neuromuscular junction. J Cell Biol 57, 315-344.

Jahn, R., and Sudhof, T. C. (1999). Membrane fusion and exocytosis. Annu Rev Biochem 68, 863-911.

Johnston, P. A., Cameron, P. L., Stukenbrok, H., Jahn, R., De Camilli, P., and Sudhof, T. C. (1989). Synaptophysin is targeted to similar microvesicles in $\mathrm{CHO}$ and PC12 cells. Embo J 8, 2863-2872.

Jorgensen, E. M., Hartwieg, E., Schuske, K., Nonet, M. L., Jin, Y., and Horvitz, H. R. (1995). Defective recycling of synaptic vesicles in synaptotagmin mutants of Caenorhabditis elegans. Nature 378, 196199.

Kittler, J. T., Delmas, P., Jovanovic, J. N., Brown, D. A., Smart, T. G., and Moss, S. J. (2000). Constitutive endocytosis of GABAA receptors by an association with the adaptin AP2 complex modulates inhibitory synaptic currents in hippocampal neurons. J Neurosci 20, 79727977.

Klopfenstein, D. R., Tomishige, M., Stuurman, N., and Vale, R. D. (2002). Role of phosphatidylinositol $(4,5)$ bisphosphate organization in membrane transport by the Unc104 kinesin motor. Cell 109, 347-358.

Lavezzari, G., McCallum, J., Dewey, C. M., and Roche, K. W. (2004). Subunit-specific regulation of NMDA receptor endocytosis. J Neurosci 24, 6383-6391.

Lazzell, D. R., Belizaire, R., Thakur, P., Sherry, D. M., and Janz, R. (2004). SV2B regulates synaptotagmin 1 by direct interaction. J Biol Chem 279, 52124-52131. 
Ledesma, M. D., Brugger, B., Bunning, C., Wieland, F. T., and Dotti, C. G. (1999). Maturation of the axonal plasma membrane requires upregulation of sphingomyelin synthesis and formation of protein-lipid complexes. Embo J 18, 1761-1771.

Lee, S. H., Liu, L., Wang, Y. T., and Sheng, M. (2002). Clathrin adaptor AP2 and NSF interact with overlapping sites of GluR2 and play distinct roles in AMPA receptor trafficking and hippocampal LTD. Neuron 36, 661-674.

Lee, S. H., Simonetta, A., and Sheng, M. (2004). Subunit rules governing the sorting of internalized AMPA receptors in hippocampal neurons. Neuron 43, 221-236.

Lin, J. W., Ju, W., Foster, K., Lee, S. H., Ahmadian, G., Wyszynski, M., Wang, Y. T., and Sheng, M. (2000). Distinct molecular mechanisms and divergent endocytotic pathways of AMPA receptor internalization. Nat Neurosci 3, 1282-1290.

Llinas, R. R., Sugimori, M., Moran, K. A., Moreira, J. E., and Fukuda, M. (2004). Vesicular reuptake inhibition by a synaptotagmin I C2B domain antibody at the squid giant synapse. Proc Natl Acad Sci U S A 101, 17855-17860.

Luscher, C., Xia, H., Beattie, E. C., Carroll, R. C., von Zastrow, M., Malenka, R. C., and Nicoll, R. A. (1999). Role of AMPA receptor cycling in synaptic transmission and plasticity. Neuron 24, 649-658.

Malenka, R. C., and Bear, M. F. (2004). LTP and LTD: an embarrassment of riches. Neuron 44, 5-21.

Malinow, R., and Malenka, R. C. (2002). AMPA receptor trafficking and synaptic plasticity. Annu Rev Neurosci 25, 103-126.

Man, H. Y., Ju, W., Ahmadian, G., and Wang, Y. T. (2000a). Intracellular trafficking of AMPA receptors in synaptic plasticity. Cell Mol Life Sci 57, 1526-1534.

Man, H. Y., Lin, J. W., Ju, W. H., Ahmadian, G., Liu, L., Becker, L. E., Sheng, M., and Wang, Y. T. (2000b). Regulation of AMPA receptormediated synaptic transmission by clathrin-dependent receptor internalization. Neuron 25, 649-662.

Marazzi, G., and Buckley, K. M. (1993). Accumulation of mRNAs encoding synaptic vesicle-specific proteins precedes neurite extension during early neuronal development. Dev Dyn 197, 115124.

Martin, S., and Henley, J. M. (2004). Activity-dependent endocytic sorting of kainate receptors to recycling or degradation pathways. Embo J 23, 4749-4759. 
Mauch, D. H., Nagler, K., Schumacher, S., Goritz, C., Muller, E. C., Otto, A., and Pfrieger, F. W. (2001). CNS synaptogenesis promoted by glia-derived cholesterol. Science 294, 1354-1357.

McMahon, H. T., and Mills, I. G. (2004). COP and clathrin-coated vesicle budding: different pathways, common approaches. Curr Opin Cell Biol 16, 379-391.

Metzler, M., Li, B., Gan, L., Georgiou, J., Gutekunst, C. A., Wang, Y., Torre, E., Devon, R. S., Oh, R., Legendre-Guillemin, V., et al. (2003). Disruption of the endocytic protein HIP1 results in neurological deficits and decreased AMPA receptor trafficking. Embo J 22, 32543266 .

Miller, K. E., DeProto, J., Kaufmann, N., Patel, B. N., Duckworth, A., and Van Vactor, D. (2005). Direct observation demonstrates that Liprin-alpha is required for trafficking of synaptic vesicles. Curr Biol 15, 684-689.

Motley, A., Bright, N. A., Seaman, M. N., and Robinson, M. S. (2003). Clathrin-mediated endocytosis in AP-2-depleted cells. J Cell Biol 162, 909-918.

Mozhayeva, M. G., Sara, Y., Liu, X., and Kavalali, E. T. (2002). Development of vesicle pools during maturation of hippocampal synapses. J Neurosci 22, 654-665.

Murthy, V. N., and De Camilli, P. (2003). Cell biology of the presynaptic terminal. Annu Rev Neurosci 26, 701-728.

Nicholson-Tomishima, K., and Ryan, T. A. (2004). Kinetic efficiency of endocytosis at mammalian CNS synapses requires synaptotagmin I. Proc Natl Acad Sci U S A 101, 16648-16652.

Nishimune, A., Isaac, J. T., Molnar, E., Noel, J., Nash, S. R., Tagaya, M., Collingridge, G. L., Nakanishi, S., and Henley, J. M. (1998). NSF binding to GluR2 regulates synaptic transmission. Neuron 21, 87-97.

Odorizzi, G., Cowles, C. R., and Emr, S. D. (1998). The AP-3 complex: a coat of many colours. Trends Cell Biol 8, 282-288.

Okada, Y., Yamazaki, H., Sekine-Aizawa, Y., and Hirokawa, N. (1995). The neuron-specific kinesin superfamily protein KIF1A is a unique monomeric motor for anterograde axonal transport of synaptic vesicle precursors. Cell 81, 769-780.

Osten, P., Srivastava, S., Inman, G. J., Vilim, F. S., Khatri, L., Lee, L. M., States, B. A., Einheber, S., Milner, T. A., Hanson, P. I., and Ziff, E. B. (1998). The AMPA receptor GluR2 $C$ terminus can mediate a reversible, ATP-dependent interaction with NSF and alpha- and betaSNAPs. Neuron 21, 99-110. 
Owen, D. J. (2004). Linking endocytic cargo to clathrin: structural and functional insights into coated vesicle formation. Biochem Soc Trans 32, 1-14.

Pearse, B. M. (1975). Coated vesicles from pig brain: purification and biochemical characterization. J Mol Biol 97, 93-98.

Praefcke, G. J., and McMahon, H. T. (2004). The dynamin superfamily: universal membrane tubulation and fission molecules? Nat Rev Mol Cell Biol 5, 133-147.

Provoda, C. J., Waring, M. T., and Buckley, K. M. (2000). Evidence for a primary endocytic vesicle involved in synaptic vesicle biogenesis. J Biol Chem 275, 7004-7012.

Ritter, B., Denisov, A. Y., Philie, J., Deprez, C., Tung, E. C., Gehring, K., and McPherson, P. S. (2004). Two WXXF-based motifs in NECAPs define the specificity of accessory protein binding to AP-1 and AP-2. Embo J 23, 3701-3710.

Rizzoli, S. O., and Betz, W. J. (2005). Synaptic vesicle pools. Nat Rev Neurosci 6, 57-69.

Rohde, G., Wenzel, D., and Haucke, V. (2002). A phosphatidylinositol $(4,5)$-bisphosphate binding site within mu2adaptin regulates clathrin-mediated endocytosis. J Cell Biol 158, 209214.

Royle, S. J., and Lagnado, L. (2003). Endocytosis at the synaptic terminal. J Physiol 553, 345-355.

Rumpel, S., LeDoux, J., Zador, A., and Malinow, R. (2005). Postsynaptic receptor trafficking underlying a form of associative learning. Science 308, 83-88.

Schafer, D. A. (2004). Regulating actin dynamics at membranes: a focus on dynamin. Traffic 5, 463-469.

Schoch, S., Deak, F., Konigstorfer, A., Mozhayeva, M., Sara, Y., Sudhof, T. C., and Kavalali, E. T. (2001). SNARE function analyzed in synaptobrevin/VAMP knockout mice. Science 294, 1117-1122.

Scott, D. B., Michailidis, I., Mu, Y., Logothetis, D., and Ehlers, M. D. (2004). Endocytosis and degradative sorting of NMDA receptors by conserved membrane-proximal signals. J Neurosci 24, 7096-7109.

Shapira, M., Zhai, R. G., Dresbach, T., Bresler, T., Torres, V. I., Gundelfinger, E. D., Ziv, N. E., and Garner, C. C. (2003). Unitary assembly of presynaptic active zones from Piccolo-Bassoon transport vesicles. Neuron 38, 237-252. 
Sheng, M., and Kim, M. J. (2002). Postsynaptic signaling and plasticity mechanisms. Science 298, 776-780.

Sheng, M., and Nakagawa, T. (2002). Neurobiology: glutamate receptors on the move. Nature 417, 601-602.

Sorkin, A. (2004). Cargo recognition during clathrin-mediated endocytosis: a team effort. Curr Opin Cell Biol 16, 392-399.

Sudhof, T. C. (2004). The synaptic vesicle cycle. Annu Rev Neurosci 27, 509-547.

Sudhof, T. C., and Rizo, J. (1996). Synaptotagmins: C2-domain proteins that regulate membrane traffic. Neuron 17, 379-388.

Takei, K., and Haucke, V. (2001). Clathrin-mediated endocytosis: membrane factors pull the trigger. Trends Cell Biol 11, 385-391.

Terashima, A., Cotton, L., Dev, K. K., Meyer, G., Zaman, S., Duprat, F., Henley, J. M., Collingridge, G. L., and Isaac, J. T. (2004). Regulation of synaptic strength and AMPA receptor subunit composition by PICK1. J Neurosci 24, 5381-5390.

Thiele, C., Hannah, M. J., Fahrenholz, F., and Huttner, W. B. (2000). Cholesterol binds to synaptophysin and is required for biogenesis of synaptic vesicles. Nat Cell Biol 2, 42-49.

Tomita, S., Stein, V., Stocker, T. J., Nicoll, R. A., and Bredt, D. S. (2005). Bidirectional synaptic plasticity regulated by phosphorylation of stargazin-like TARPs. Neuron 45, 269-277.

Walther, K., Diril, M. K., Jung, N., and Haucke, V. (2004). Functional dissection of the interactions of stonin 2 with the adaptor complex AP-2 and synaptotagmin. Proc Natl Acad Sci U S A 101, 964-969.

Wang, Y. T., and Linden, D. J. (2000). Expression of cerebellar longterm depression requires postsynaptic clathrin-mediated endocytosis. Neuron 25, 635-647.

Winckler, B., and Mellman, I. (1999). Neuronal polarity: controlling the sorting and diffusion of membrane components. Neuron 23, 637640.

Zhai, R. G., Vardinon-Friedman, H., Cases-Langhoff, C., Becker, B., Gundelfinger, E. D., Ziv, N. E., and Garner, C. C. (2001). Assembling the presynaptic active zone: a characterization of an active one precursor vesicle. Neuron 29, 131-143. 


\section{Lebenslauf}

Kathrin Kastning

geboren am 06. Dezember 1975

in Stadthagen

\section{Schulbildung:}

1987 bis 1995

Gymnasium Bad Nenndorf mit Abitur

\section{Studium:}

1995 bis $2000 \quad$ Studium der Biochemie an der Universität Bielefeld

2000

Diplom in Biochemie an der Universität Bielefeld

Diplomarbeit in Entwicklungsbiologie in der Arbeitsgruppe Prof. Dr. H. Jockusch: "Expression des Neuregulin-1 Gens und der ErbB-Rezeptoren in etablierten Zellinien und im Mausmodell für neurodegenerative Erkrankungen."

Mündliche Diplomprüfung in den Fächern Biochemie, Gentechnologie, Bioanorganische Chemie und Proteinaufarbeitung

\section{Promotionsstudium}

2001 bis $2004 \quad$ Doktorarbeit in der Nachwuchsgruppe Dr. V. Haucke des SFB 523 an der Universität Göttingen "Recognition of basic sorting motifs within synaptic membrane cargo proteins by the clathrin-adaptor complex AP-2"

2004 bis 2005 Fortsetzung der Doktorarbeit in der Arbeitsgruppe Prof. Dr. V. Haucke am Institut für Chemie und Biochemie der Freien Universität Berlin

Stipendiatin im Graduiertenkolleg 521 der Universität Göttingen „Intrazelluläre Transportprozesse von Makromolekülen"

Göttingen, den 23. Mai 2005

Kathrin Kastning 